\title{
The Photochemistry of Organic Materials for Photonic Devices
}

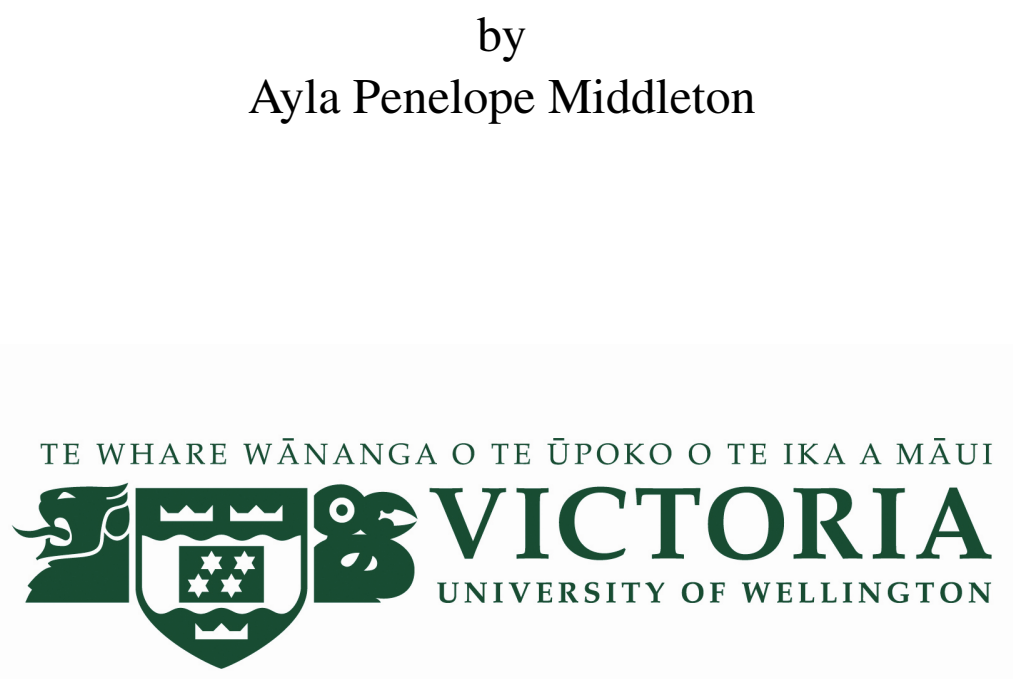

\author{
A thesis \\ submitted to School of Chemical and Physical Sciences \\ Victoria University of Wellington \\ in fulfilment of the \\ requirements for the degree of \\ Masters \\ in Chemistry.
}

School of Chemical and Physical Sciences

Victoria University of Wellington

31st March 2014 


\section{Abstract}

Optically active organic chromophores have attracted much interest in recent years for their potential for use in photonic devices. Chromophores such as compound (1) have been found to have a very high second order nonlinear susceptibility ( $\beta$ ) value of $650 \times$ $10^{-30}$ esu in dimethyl formamide. ${ }^{1}$ The performance of 1 in a polymer film is much lower than this due to the formation of aggregates which hinder the poling process necessary to ensure a noncentrosymmetric arrangement of the molecules in order to display second order nonlinear behaviour.

The molecular aggregation behaviour of a set of second order nonlinear compounds based on compound 1 have been studied in this thesis. These compounds share the backbone shown in figure 1 with pendant groups added to the $R_{1} R_{2}$ and $R_{3}$ positions, with the aim of finding substituent groups that can be added to the optically active merocyanine backbone that reduce the aggregation and increase the solubility of the compounds. This in turn will make them more suitable for use in photonics devices.

It was found that a $\mathrm{C}_{11} \mathrm{H}_{23}$ alkyl chain added to the $\mathrm{R}_{3}$ position made the largest contribution to decreasing aggregation. Bulky groups on the $\mathrm{R}_{1}$ and $\mathrm{R}_{2}$ positions also reduced aggregation. As a result compounds 5 and 8 , with $\mathrm{R}_{3}=\mathrm{C}_{11} \mathrm{H}_{23}$ and bulky groups attached displayed the least aggregation of the compounds studied.

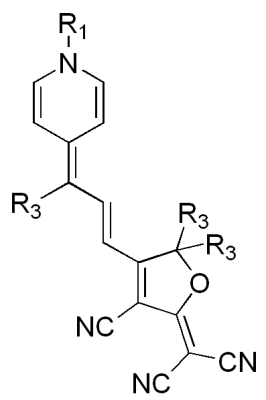

Figure 1 Merocyanine backbone with substituent postions marked 


\section{Acknowledgements}

I would like to thank the members of the I.R.L Photonics group for their help and support. Especially Dave Clarke, Ashraf Moideen, and Bob Breukers for the synthesis of materials for me. I would also like to thank my supervisors Andy Kay and Gerald Smith.

Victoria, Tra Me, Stefaan, Adam, Dave, Bob, Jack, and Damian, thank you for making IRL a great place to work. I could never have finished without the support of my friends and family and for that I especially thank my Parents and Craig. 


\section{Contents}

Abstract

Acknowledgements ii

Table of Contents iii

1 Introduction 1

1.1 General Introduction $\ldots \ldots \ldots \ldots$

1.2 Nonlinear Optics . . . . . . . . . . . . . . . . . . 2

1.2.1 Theoretical Basis of Nonlinear Optics . . . . . . . . . . . . . 2

1.2.2 Second Order Nonlinear Optical Effects . . . . . . . . . . 3

1.3 Materials for Second-Order Nonlinear Optical Devices . . . . . . . . 5

1.3.1 Traditional Materials for NLO Devices . . . . . . . . . . . 5

1.3.2 Organic materials for Second Order NLO Devices . . . . . . 6

1.4 Integration of NLO chromophores into a bulk material $\ldots \ldots$. . . . 8

1.4.1 Methods of preparing samples ............ 8

1.4 .2 Aggregation ...................... 11

2 Research Aims 13

3 UV-Vis Spectroscopic Studies $\quad 18$

3.1 Concentration Dependant Absorption Spectra . . . . . . . . . . . . . 18 
3.1 .1 Introduction . . . . . . . . . . . . . . . 18

3.1.2 Concentration Dependant Absorption Results . . . . . . . . . . . 20

3.2 Solvatochromism . . . . . . . . . . . . . . . 32

3.2 .1 Introduction . . . . . . . . . . . . . . 32

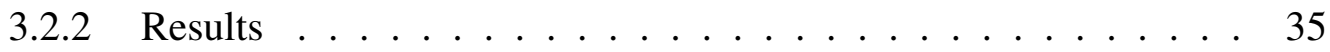

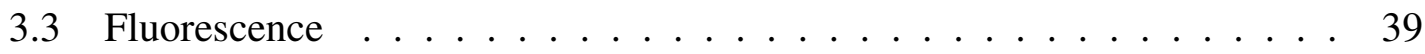

3.3.1 Introduction . . . . . . . . . . . . . . 39

3.3 .2 Results ....................... 43

4 Detection of Rotamers by NMR

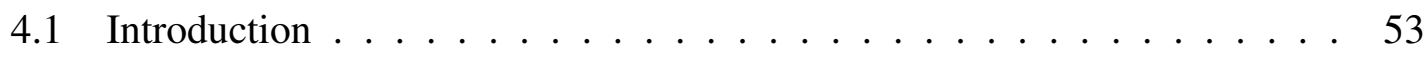

4.2 Results . . . . . . . . . . . . . . . . . . 54

5 Discussion $\quad 56$

5.1 Concentration dependent UV-Vis Studies . . . . . . . . . . . 57

5.2 Fluorescence . . . . . . . . . . . . . . . . . 60

5.3 Rotamers ............................. 63

5.4 Solvatochromism . . . . . . . . . . . . . . . 65

$\begin{array}{lll}6 & \text { Experimental } & 70\end{array}$

$6.1 \quad$ UV-Vis Absorption Spectroscopy . . . . . . . . . . . . . . . . 70

6.2 Steady-State Fluorescence Spectroscopy . . . . . . . . . . . . . . . 71 
6.3 Fluorescence Lifetime Measurements _ . . . . . . . . . . . . . . 72

6.4 Quantum Yield Measurements . . . . . . . . . . . . . . . 72

7 Conclusions and Future Work $\quad 74$

7.1 Conclusions . . . . . . . . . . . . . . . . 74

7.2 Future Work . . . . . . . . . . . . . . . 75

References 


\section{Chapter 1}

\section{Introduction}

\subsection{General Introduction}

Since the development of fiber-optic technology, the potential of photons as carriers of information has been apparent, especially in the telecommunications industry where the ability to carry large quantities of information with high speed and minimal loss of signal is important. ${ }^{2,3}$ Photonic devices that use light as the primary signal carrier rather than traditional electronic signal transmission offer several advantages, namely that: $a$ ) data transmission speeds are faster; $b$ ) signals are not prone to electromagnetic interference; $c$ ) signal losses due to heat generation are very low; $d$ ) broader bandwidth are available; and $e$ ) it is possible for several different wavelengths to be sent through the same waveguide without causing interference or cross-talk - a phenomenon known as dense wavelength division multiplexing. ${ }^{4-7}$

A key factor in the emergence of commercial photonic devices is the development of materials that can be used in fibre-optic cables and wave-guides to not only transmit light, but allow for optical signal processing. ${ }^{8}$ Optically active materials allow for photonic devices that can perform operations such as optical switching and signal modulation without having to convert an optical signal into an electronic one and back again for further transmission. Therefore, there is a growing interest in developing materials with interesting optical properties that can perform these operations.

For photoactive materials to be successfully integrated into optical devices, the materials used for device manufacture need to not only have the necessary optical properties, but must also be suitable for integration into the device. For example, a component forming part of the waveguide (path through which the optical signal travels) must have its refractive index matched to other parts of the waveguide so that light travels through without loss due to reflection. ${ }^{9}$ Waveguide materials must also be suitable for processing and integration with other device components. ${ }^{10-12}$ These materials need to 
meet a number of performance parameters. For example they need to be photochemically stable as photonic devices are exposed to high energy light over their operational lifetime. Further more materials that are air or water sensitive or have a limited thermal stability are not desirable in commercial applications because this reduces their operational lifetime. Consequently developing photonic materials and devices that are durable remains a key challenge for researchers.

In the past two decades optically active organic compounds have attracted significant interest for their potential use in nonlinear optical (NLO) devices. ${ }^{2-8,10-14}$ This thesis will investigate the photochemistry and photophysics of organic chromophores which display high second order nonlinear susceptibilities and photochromic behaviour. The optical properties of the chromophores are important for the creation of photonic devices due to the large changes in the refractive indices of the materials that can be achieved when these chromophores are exposed to light or an electric field.

\subsection{Nonlinear Optics}

\subsubsection{Theoretical Basis of Nonlinear Optics}

The interaction of an electric field with a molecule results in a change in the polarisation of the molecule and this is the origin of the nonlinear optical response. A molecule consists of collection of positively charged nuclei surrounded by a negatively charged electron cloud. An electric field applied to the molecule will interact with the charged particles and the electron cloud will respond faster to an external electric field than the heavier nucleus resulting in an induced charge separation. Light is an oscillating electric field and so the electron distribution of the molecule will respond in an oscillatory motion. When the electric field is small, the electron charge cloud motion will also be small and will, to a good approximation, oscillate harmonically with the electric field. If the electromagnetic interaction is large, the electron cloud cannot respond linearly and the oscillation becomes anharmonic. $^{15}$

The nonlinear polarization response at the molecular level is described by the Taylor 
expansion given in eq 1.1. $P$ is the polarization of the material, $E$ is the applied electric field, and $\alpha$ is the linear polarizability $\beta$, is the first hyperpolarizability and $\gamma$ is the second hyperpolarizability. ${ }^{16}$

$$
P=\alpha E+\beta E^{2}+\gamma E^{3}+\cdots
$$

Equation 1.2 gives an equivalent equation used when the polarity of the macromolecular environment is being considered. Where $\chi^{(1)}, \chi^{(2)}$, and $\chi^{(3)}$ represent the first- secondand third-order nonlinear susceptibilities respectively. ${ }^{17}$

$$
P=\chi^{(1)} E+\chi^{(2)} E^{2}+\chi^{(3)} E^{3}+\cdots
$$

For small electric fields, where the change in polarisation is linear, $\alpha$ is the dominant term and polarisation can be satisfactorily modelled by the equation $P=\alpha E$. When a large enough electrical field is applied the $2^{\text {nd }}$ and $3^{\text {rd }}$ order nonlinear terms $\beta$ and $\gamma$ of the expansion become significant and nonlinear optical effects are apparent. ${ }^{13}$ It is important to note that as the coefficient $\beta$ is a multiplier of $E^{2}$, an even power term, it means that for a material to display a second-order nonlinear effect it must be noncentrosymmetric. If the material is centrosymmetric then the dipoles cancel and no second-order effect is observed. However for third-order nonlinear materials, $\gamma$ is a coefficient of $E^{3}$ and so third order effects have no symmetry restrictions. ${ }^{18}$ Some important nonlinear optical effects and applications of these effects are described below. Nonlinear effects are important for the manipulation of light and as such there is much interest in materials with these properties, particularly in optical communications.

\subsubsection{Second Order Nonlinear Optical Effects}

Sum-frequency generation uses the mixing of two incident light waves $\left(\omega_{1}, \omega_{2}\right)$ to create a new output beam $\left(\omega_{3}\right)$ with $\omega_{3}=\omega_{1}+\omega_{2}$. Second harmonic generation is a special case of sum-frequency generation where only one incident frequency is present, resulting in an 
output of $2 \omega$. The mixing of the waves can be represented by the nonlinear polarization in equation 1.3. Sum-frequency generation and second harmonic generation are frequently used to extend the available output of a laser system to wavelengths other than the fundamental wavelength.

$$
P^{\omega_{3}}=\epsilon_{0} \chi^{(2)}\left(-\omega_{3}, \omega_{1}, \omega_{2}\right) E^{\omega_{1}} E^{\omega_{2}}
$$

The electro-optic effect is the change in the refractive index of a noncentrosymmetric material that occurs when an electric field is applied to the material. The change in the refractive index can be described by equation 1.4 , where $n$ is the refractive index, $\mathrm{r}$ is the electro-optic coefficient of the material, and $\mathrm{E}$ is the applied electric field. ${ }^{19}$

$$
\Delta n \cong \frac{-n^{3} r E}{2}
$$

The electro-optic effect is useful in a range of applications as an external electric field can be applied to the nonlinear material, resulting in a change in the optical properties of the section of material where the electric field is applied. This allows for the manipulation of light passing through a waveguide made of the nonlinear material, an important property that permits the creation of devices such as optical signal modulators. Figure 1.1 shows a typical Mach-Zhender configuration, where the light path is split into two at (a) and then recombined at (b). After (a) light continues to travel through one path with unaltered speed, while the nonlinear medium in other path has an electric field applied to it. ${ }^{20}$ The resulting change in the refractive index of the waveguide material in this arm of the device alters the speed of light and hence the time it takes the light to reach the end of the guide, where the two paths are recombined. Depending on the difference in the speed of the two light beams their relative phases may be different and the resulting constructive or destructive interference can be used to modulate the light signal. Thus, in the event that no electric field is applied to the NLO material the speeds of the two light beams will be the same and there will be a constructive recombination. Conversely, when an electric field is applied the beam passing through the arm containing the NLO material will be retarded to such an extent that the recombination will be destructive. ${ }^{21}$ 


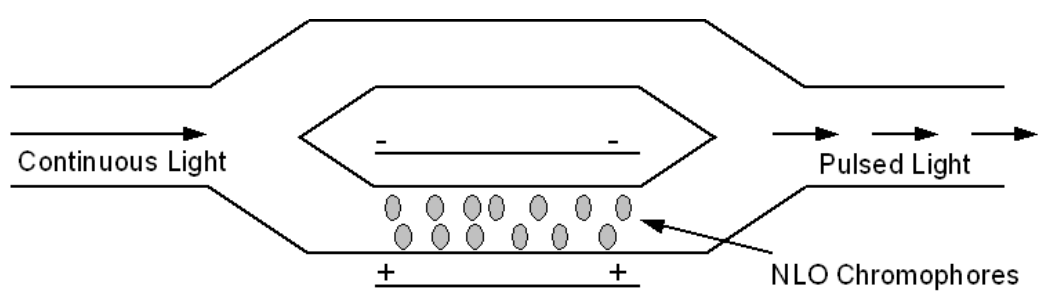

Figure 1.1 The layout of a Mach-Zhender modulator. Continuous light enters the modulator on the left and is split into two light paths. If an electric field is applied the destructive interference results in a modulated light beam output.

Organic chromophores displaying high values of the first nonlinear hyperpolarizability coefficient, $\beta$, have attracted the interest of researchers due to their potential use in photonic devices such as optical modulators, tunable Bragg gratings, and all optical switches. $^{22}$

\subsection{Materials for Second-Order Nonlinear Optical Devices}

\subsubsection{Traditional Materials for NLO Devices}

Lithium niobate $\left(\mathrm{LiNbO}_{3}\right)$ is currently the principal NLO material used in commercial applications. ${ }^{20}$ However the use of such inorganic crystals is expensive due to the difficulty of growing crystals of sufficient quality for optical applications. As an alternative, organic NLO compounds can be synthesised with relative ease, and as they are soluble in organic media, techniques such as spin processing of thin polymer film matrices can be employed in the fabrication of nonlinear optical devices. ${ }^{4}$ The host polymer refractive index can be adjusted to match other materials, allowing integration of the polymer with waveguide materials or semiconductors. Use of a polymeric material also allows for the deposition on the NLO material onto a range of substrates, including silica waveguides and semiconductor electronics. While crystalline lithium niobate can be integrated into the optical system, this requires use of separate optical and electronic modules connected by cables. ${ }^{23}$

Organic NLO materials can outperform $\mathrm{LiNbO}_{3}$ in terms of several measures of NLO performance. $\mathrm{LiNbO}_{3}$ has an electro-optic (EO) coefficient of $31 \mathrm{pm} / \mathrm{V}$ while NLO 


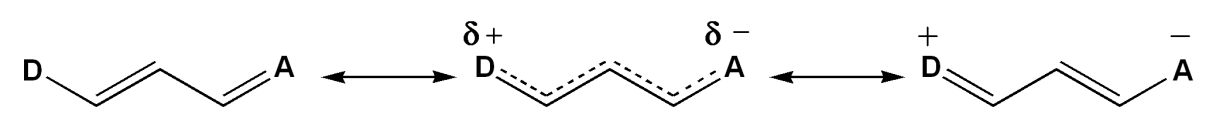

Figure 1.2 The neutral (left) and zwitterionic (right) states of a $\mathrm{D}-\pi-\mathrm{A}$ molecule. The intermediate resonant state is shown in the centre diagram.

polymers typically have values $>80 \mathrm{pm} / \mathrm{V}^{9}$ and the highest performing polymers can attain values $>300 \mathrm{pm} / \mathrm{V}$. ${ }^{1}$ This larger electro-optic response means that NLO devices can be made to operate using lower drive voltages. Lithium niobate modulators require a minimum drive voltage of around $5 \mathrm{~V}$, whereas polymeric modulators have been produced with drive voltages of less than $1 \mathrm{~V} .{ }^{9}$ Polymeric materials have longer dephasing lengths, so that at $100 \mathrm{GHz}$ operation a polymeric modulator can be $2 \mathrm{~cm}$ long while a $\mathrm{LiNbO}_{3}$ modulator is limited to a pathlength of $1 \mathrm{~mm} .^{24}$

\subsubsection{Organic materials for Second Order NLO Devices}

At a macroscopic level, organic materials for second order nonlinear devices must be noncentrosymmetric because of the symmetry restraints of the $\chi^{(2)} E^{2}$ tensor. As a result, research into optimising $\beta$ in organic materials has focused on "push-pull" molecules consisting of an electron donating group at one end of the molecule (providing electron "push") and an acceptor group at the other (electron "pull"), bridged by a conjugated interconnect. This results in a highly polarisable molecule due to facile electron delocalisation and mobility under an electric field through the conjugated $\pi$ orbital system.

The ground state of these molecules can be regarded as a mixture of two states, an electrically neutral state where there is no electron transfer between the donor and acceptor (the leftmost structure in figure 1.2); and a zwitterionic state where the donor group has completely transferred an electron to the acceptor group (rightmost structure in figure 1.2). Most commonly, the ground state lies between these two extremes and the actual intermediate resonant state is shown in the centre of figure 1.2. ${ }^{19}$ The degree of charge separation from the electrically neutral state is described by the bond-lengthalternation parameter (BLA), a measure of the difference between the average lengths of adjacent carbon-carbon single and double bonds in the polymethine chain. ${ }^{25}$ 
BLA becomes an important factor in establishing structure-function relationships as the magnitude of second-order nonlinear effects depend on the size of the first non-linear hyperpolarizability coefficient, $\beta$, and on the dipole moment, $\mu$, both of which are related to the extent of BLA. ${ }^{26}$ The dependence of $\beta$ and $\mu$ on BLA is shown in figure 1.3. It is apparent from this figure that there are two zones where $\mu$ and $\beta$ combine to give a large molecular second order NLO "figure of merit", $|\mu . \beta|$. One zone has negative BLA and chromophores with this structure are considered left-hand-side (LHS) molecules. The other zone occurs when BLA is positive and $\beta$ is strongly negative as $|\mu . \beta|$ is an absolute value. Chromophores that lie in this region of BLA are known as right-hand-side (RHS) molecules.

Optimisation of $|\mu . \beta|$ can be achieved by increasing the donor or acceptor strength or by elongation of the length of the conjugated bridge. Elongating the conjugation length of the bridge results in a reduction of the energy gap between the ground and excited states of the molecule, thereby increasing $\beta$. Changing the electron donor or acceptor strengths allows fine tuning of where the ground state of the molecule lies, i.e. between the neutral or zwitterionic states (figure 1.2) so that the degree of BLA can be controlled. Examples of second order molecules that have been synthesised are shown in figure 1.4. It is seen that compound (a) with an aniline donor and nitro acceptor has a modest figure of merit of $580 \times 10^{-48}$ esu. This compares to compound (f) where it is seen that the presence of the strong isoxazalone acceptor group along with an increase in the polyene interconnect length leads to the realization of compounds with higher figure of merit, i.e. greater than $8000 \times 10^{-48}$ esu. $^{1}$

LHS and RHS molecules have several differences in the way they behave. LHS molecules with large $\beta$ values also exhibit a reasonable degree of negative BLA with a ground state that is approaching a neutral species and a charge separated excited state. ${ }^{4}$ As a result these molecules show positive solvatochromism; their ground state is destabilized in more polar solvents and so the absorption spectra exhibit a bathochromic shift. ${ }^{27}$ RHS molecules, in contrast, have much more zwitterionic character in their ground state and exhibit hypsochromic shift in solvents of increasing polarity. While much research has been done on LHS chromophores ${ }^{2,9,10,21,28-31}$ there have been comparatively few studies of the RHS molecules. The merocyanines studied in this thesis are RHS chromophores 


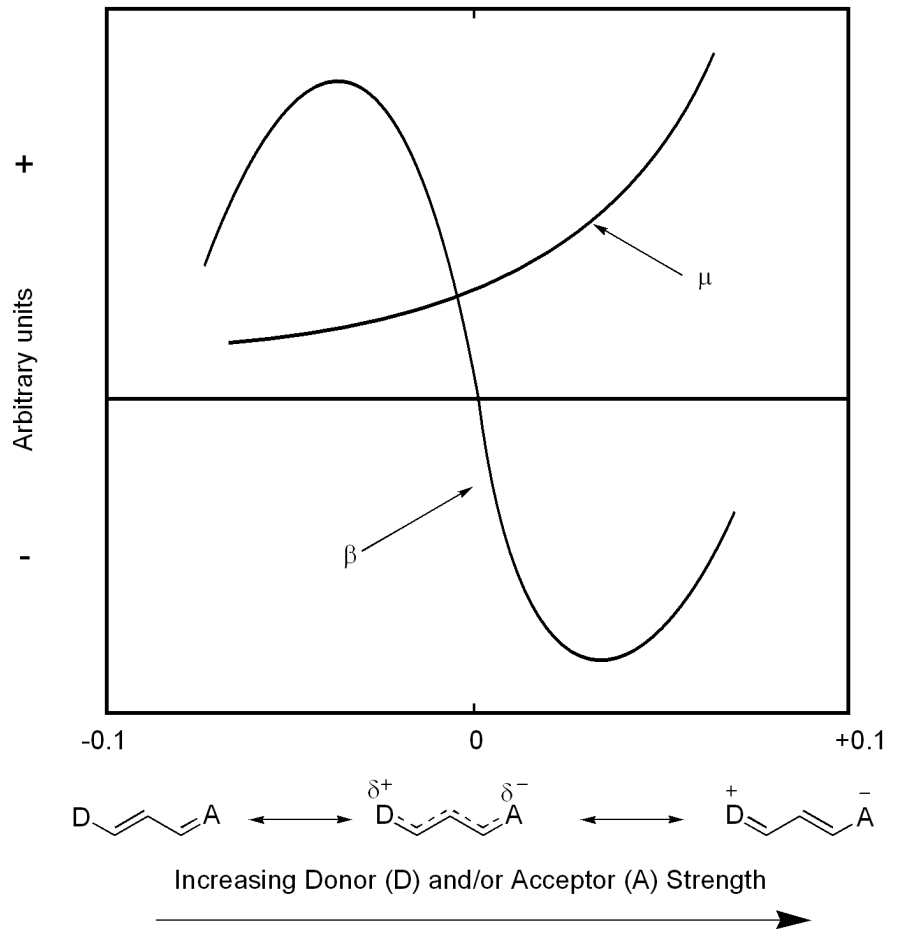

Figure 1.3 Graph showing the dependence of $\beta$ and $\mu$ (in arbitrary units) on BLA.

that are described in detail in the research aims chapter 2.

\subsection{Integration of NLO chromophores into a bulk mater- ial}

\subsubsection{Methods of preparing samples}

While maximising $|\mu . \beta|$ on the molecular scale should give compounds with a high electro-optic response, for these compounds to be usefully integrated into bulk materials, other properties must be considered for the compounds to be viable for use in nonlinear optical devices. Importantly, if the alignment of the chromophores is centrosymmetric then the second-order nonlinear optical effects, being vector quantities, cancel and the net change in polarizability is zero. ${ }^{17}$

There are various methods of preparing NLO materials so that a noncentrosymmetric arrangement is obtained. It is possible to grow crystals containing organic NLO 


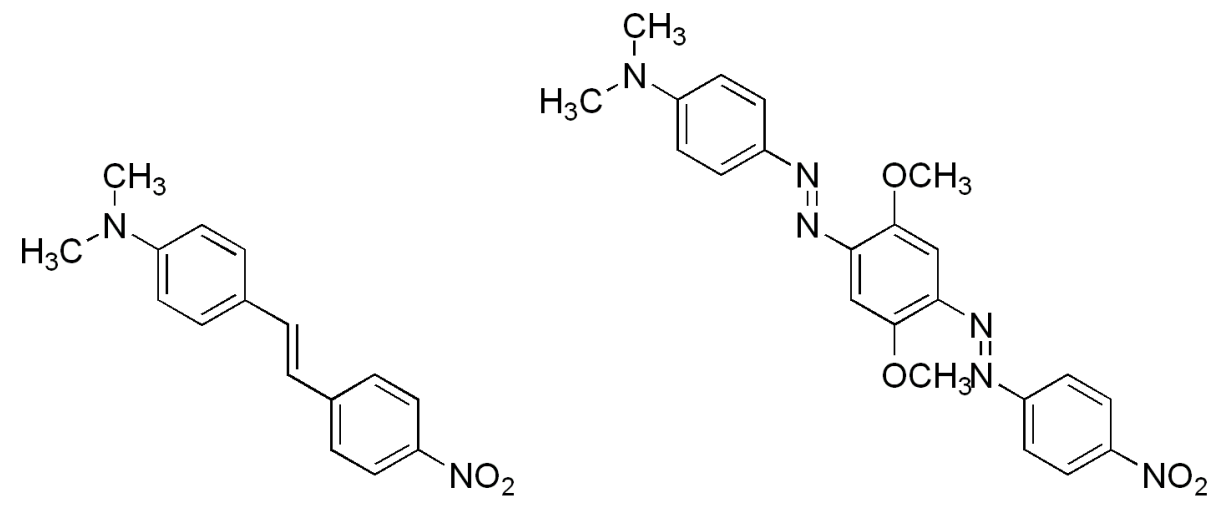

(a) $|\mu . \beta|=580 \times 10^{-48}$ esu $^{32}$

(b) $|\mu \cdot \beta|=1040 \times 10^{-48} \mathrm{esu}^{33}$
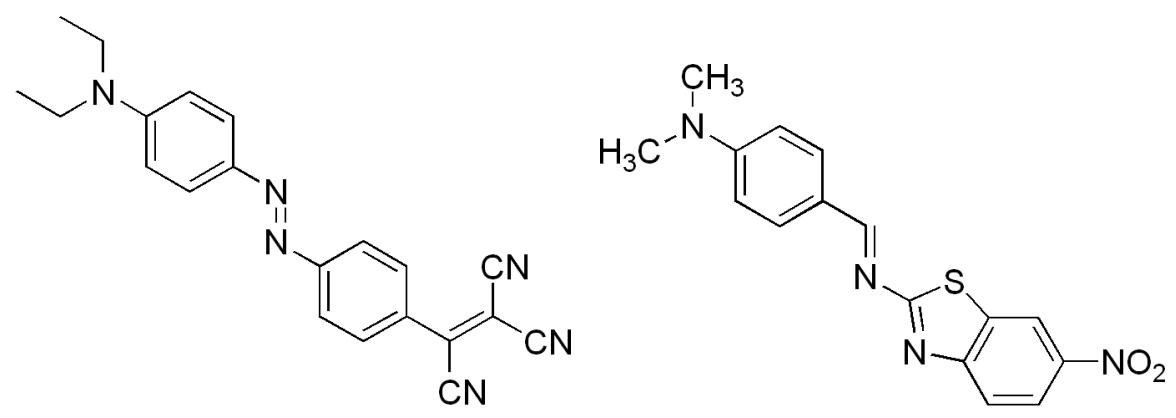

(c) $|\mu \cdot \beta|=4100 \times 10^{-48} \mathrm{esu}^{33}$

(d) $|\mu \cdot \beta|=640 \times 10^{-48} \mathrm{esu}^{34}$
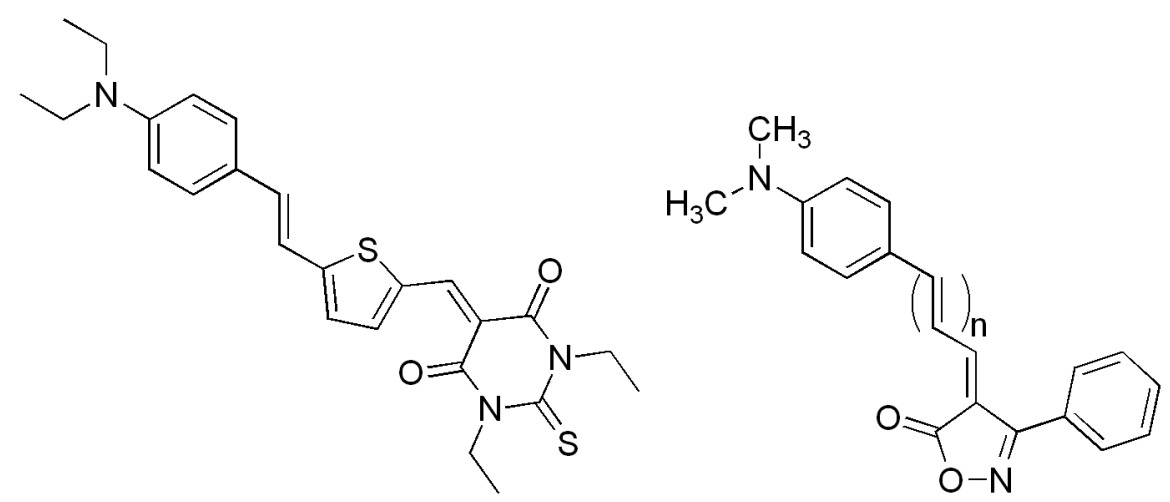

(e) $|\mu \cdot \beta|=2400 \times 10^{-48} \mathrm{esu}^{34}$

(f) $n=0,|\mu . \beta|=312 \times 10^{-48} \mathrm{esu}$; $n=3,|\mu . \beta|=8171 \times 10^{-48} \mathrm{esu}^{35}$

Figure 1.4 Selected Second Order NLO Chromophores 
chromophores that have inherent noncentrosymmetry; however it is often difficult to obtain crystals of the requisite quality and size, and while noncentrosymmetry has been observed in organic crystals this is a somewhat unpredictable process and is therefore difficult to control. ${ }^{10}$ There is also difficulty in integrating such crystals into a waveguide.

An alternative to organic crystals is to use a polymer based matrix by producing a polymer containing the chromophore; which can be readily achieved by covalently attaching the chromophore to a polymer backbone, for example by grafting a chromophore containing an alcohol tethering group to an acid containing polyimide. Or by incorporating the chromophore as a dopant in a host-guest polymer matrix. Host-guest matrices allow straightforward integration of the chromophore into a polymer environment allowing for rapid assessment of macroscopic response. A particular advantage of host -guest systems is that the chromophore and polymer can be dissolved in a solvent and then applied to a substrate by methods such as spin coating. The use of host-guest matrices is a popular technique as processing is relatively straightforward and cost-effective, both of which are important for the development of commercial non-linear devices.

The methods of preparing the polymer sample, such as spin-processing to create a thinfilm, result in a random arrangement of chromophore molecules. An additional step of 'poling' with a strong electric field is required to align the dipoles of the chromophores within the matrix. ${ }^{36}$ In this process, the polymer film containing the NLO chromophores is heated above the polymer's glass transition temperature $\left(\mathrm{T}_{\mathrm{g}}\right)$. Above this temperature molecular rotation and realignment is possible. An intense electric field (approximately $100 \mathrm{~V} /$ micron for contact poling) is then applied across the film, and the chromophore molecules will rotate so their dipoles are aligned with the field. The polymer is cooled, while still applying the electric field to retain molecular alignment so the chromophores will be locked in an ordered array below the glass transition temperature. ${ }^{37,38}$ The ideal result is shown in figure 1.5(b) which demonstrates a polymer matrix with all chromophore dipoles aligned parallel to each other.

The measured NLO responses in polymer films are routinely lower than the expected calculated values. This is because after the poling field has been removed, thermal relaxation will result in some chromophore molecules rotating away from the aligned 


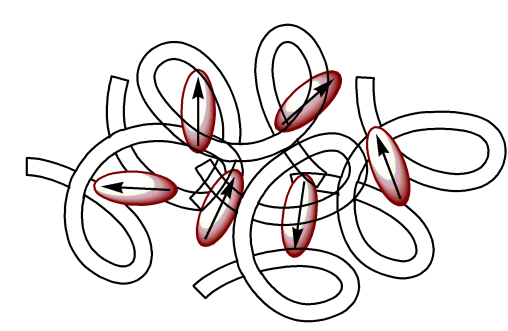

(a) random dipole alignment

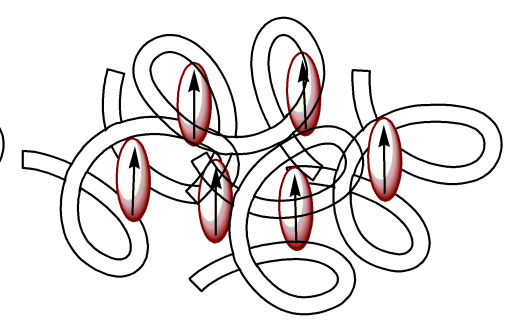

(b) aligned dipoles after polling

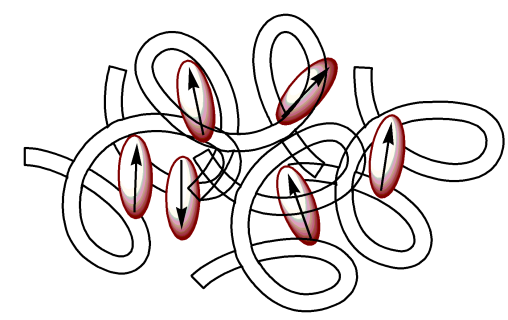

(c) realistic dipole arrangement after poling

Figure 1.5 Alignment of chromophore dipoles in a host-guest polymer matrix

direction, reducing the observed NLO effect, a situation shown in figure 1.5(c). As the chromophores tend to have high dipole moments there are also strong columbic interactions between neighbouring chromophore molecules, which can result in the formation of aggregates. These aggregates also reduce the NLO effects as it means the chromophores are aligned antiparallel to each other meaning that poling efficiency is decreased due to the strong dipole-dipole interactions.

\subsubsection{Aggregation}

Aggregation is a major limitation in the application of organic NLO host-guest systems. Due to their $\mathrm{D}-\pi-\mathrm{A}$ nature, the chromophores typically have a high dipole moment. This means that the chromophores are prone to coulombically interacting with each other, leading to aggregation. ${ }^{5}$ This is especially a problem at the high concentrations used in think films. ${ }^{31}$ An aggregate is an extended network of molecules that are closely associated with each other due to specific interactions via coulombic, van der Waals forces or H-bonding between the chromophores. An aggregate can form in several geometries, and the shape of the individual molecules will affect how this aggregation will occur. ${ }^{39}$ The $\mathrm{D}-\pi-\mathrm{A}$ conjugated system required between donor and acceptor groups leads 


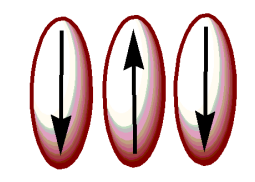

(a) H-aggregate

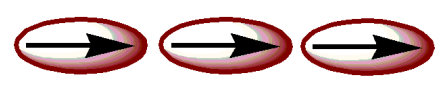

(b) J-aggregate

Figure 1.6 The arrangement of molecules within $\mathrm{H}$ - and J- aggregates.

to prolate (i.e. cigar-shaped) molecules and this shape can promote the formation of aggregates, as a flat polar molecule can easily stack with other similar molecules.

The two common aggregate configurations encountered with organic chromophores are $\mathrm{H}$ - and J-aggregates. These are one dimensional aggregates where the molecules are arranged in an antiparallel stacking arrangement for H-aggregates shown in figure 1.6(a), or in a parallel "head-to-tail" alignment in the case of a J-aggregate (figure 1.6(b)). ${ }^{40}$ These are both seen in merocyanine dyes. ${ }^{41,42}$ 


\section{Chapter 2}

\section{Research Aims}

As has been discussed in the introduction, a major challenge in the use of organic chromophores in NLO devices is maintaining the desired optical properties of the chromophore in the conditions necessary for the integration of the chromophore within the NLO device. For use in a photonic device the chromophore needs to be incorporated into a polymer matrix and this is typically achieved through the use of a host-guest polymer system or through covalent attachment of the chromophore to a polymer backbone. Two of the most important considerations is therefore for the chromophores to be sufficiently soluble to allow processing as thin polymer films and for them to have high thermal stabilities, i.e. $\mathrm{T}_{\mathrm{d}}>220^{\circ} \mathrm{C}$. Both of these parameters are critical for the process of electric field poling which is used to induce noncentrosymmetry at the macroscopic level. As well of course a chromophore displaying a large NLO effect is also desired.

However, the experimentally observed electro-optic response NLO polymer materials is often lower than that expected in thin films, and often a large molecular NLO response $(\beta)$ does not necessarily translate to a large macromolecular $\chi^{(2)}$ response. This is because of the formation of centrosymmetric aggregates that cancel out the first hyperpolarizability vector of individual molecules.

The merocyanine compound 1 shown in figure 2.1 has been found to have a high measured $\beta$ value of $650 \times 10^{-30}$ esu in dimethyl formamide. ${ }^{1}$ This compound has been chosen as the base structure in this research as the high $\beta$ values are an important property in selecting compounds to use in photonic devices as a large figure of merit $(|\mu . \beta|)$ is desirable for the largest possible electro-optic response of the device.

Preliminary studies have shown that these merocyanines have a very strong tendency to aggregate in nonpolar environments similar to those found in polymers. A series of substituted merocyanines have been synthesised to try and reduce this aggregation and to elucidate the effectiveness of these strategies of preventing aggregation.

Figure 2.2 shows the basic merocyanine backbone with the four positions where 


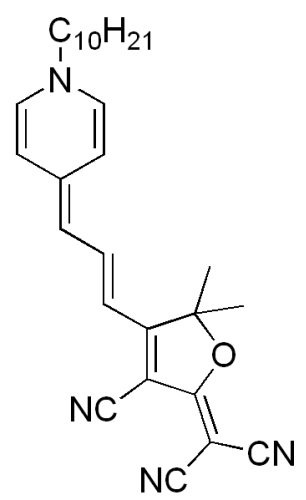

Figure 2.1 Compound 1

subsitiuent groups have been added. The basic merocyanine in figure 2.2 with no substituent groups, (i.e. with $\mathrm{R}_{1}=\mathrm{CH}_{3}, \mathrm{R}_{2}=\mathrm{H}, \mathrm{R}_{3}=\mathrm{CH}_{3}$ ) is unsuitable for use in devices due to its low solubility in solvents for host polymers required to cast films of material so most work has been performed on molecules with $\mathrm{R}_{1}=\mathrm{C}_{10} \mathrm{H}_{21}$, which have better solubilities in organic solvents. ${ }^{43}$ An important consideration in preventing aggregation of the merocyanine is the molecule's shape. The unsubstituted merocyanine is roughly planar and prolate in shape, that is, if the molecule's poles are the $\delta+$ and $\delta$ - donor and acceptor groups respectively, then the molecular dimension is greater from pole to pole than across its equator. It is expected that by adding bulky substituent groups around the molecule to make it more oblate in shape, i.e. with an equator dimension greater than its pole to pole dimension, then molecular dimerisation will be reduced as the bulky groups will provide steric hindrance between the $\delta+$ and $\delta$ - groups. The bulky groups have also been chosen with subsitiuent arene rings with the aim of forcing stacking of the molecules via $\pi-\pi$ stacking of the bulky groups. ${ }^{44,45}$ This stacking has been demonstrated in the crystal structure obtained for the tricyano acceptor group containing two phenyl rings (figure 2.3). It can be seen that the phenyl rings interact strongly with each other and largely determine the stacking of the unit cell. ${ }^{46}$

The compounds to be studied in this research programme are based on the merocyanine basic structure shown in figure 2.2. The groups $R_{1}-R_{3}$ are the positions where bulky groups have been added to the molecule in various substitution patterns. The structures of these substituted compounds are summarised in table 2.1, and the abbreviations for each bulky group are shown in figure 2.4 . 


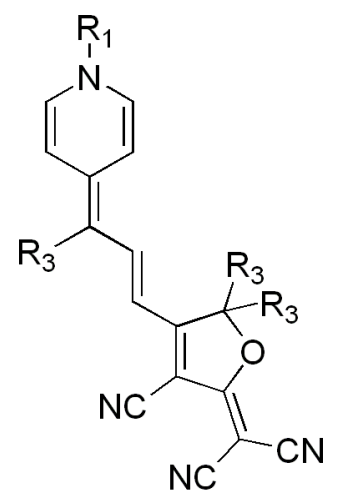

Figure 2.2 Basic merocyanine backbone to be studied

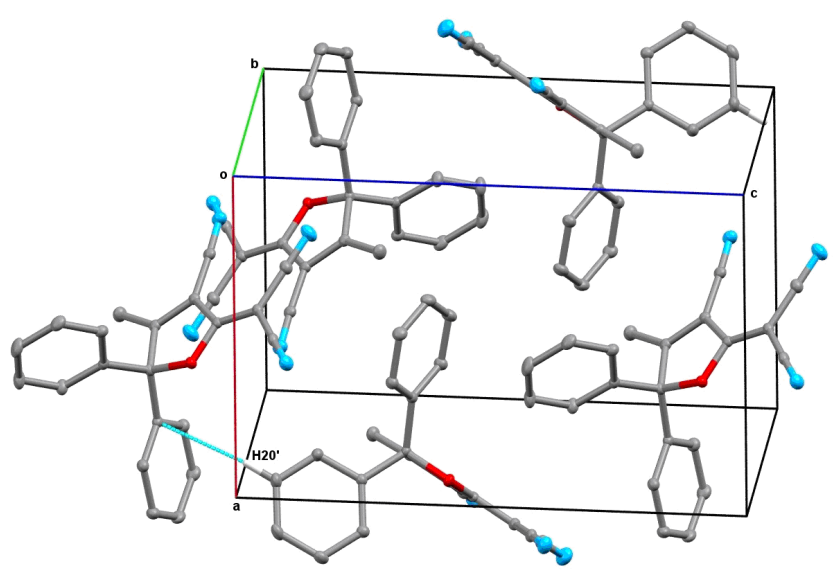

Figure 2.3 Unit cell showing hydrogen bonding from phenyl ring to phenyl ring, illustrated with a blue dashed line ${ }^{46}$ 
These RHS chromophores exhibit significant solvatochromism in their UV-Vis absorption and fluorescence spectra as the dyes ground state is affected by the surrounding environment. An aggregate of the chromophore also has a different absorption spectra than the chromophore monomer in isolation, so spectroscopy is an ideal way of monitoring changes in aggregation. This thesis will examine the chromophores described above (and shown in table 2.1) to determine the effectiveness of the addition of the bulky groups to the basic merocyanine backbone in preventing aggregation.

Table 2.1 Merocyanines studied in this work

\begin{tabular}{|c|ccc|}
\hline Compound & $\mathrm{R}_{1}$ & $\mathrm{R}_{2}$ & $\mathrm{R}_{3}$ \\
\hline $\mathbf{1}$ & $\mathrm{C}_{10} \mathrm{H}_{21}$ & $\mathrm{H}$ & $\mathrm{CH}_{3}$ \\
$\mathbf{2}$ & $\mathrm{C}_{10} \mathrm{H}_{21}$ & $\mathrm{H}$ & $\mathrm{C}_{11} \mathrm{H}_{23}$ \\
$\mathbf{3}$ & $\mathrm{C}_{10} \mathrm{H}_{21}$ & $\mathrm{H}$ & $\mathrm{PhOBz}$ \\
\hline $\mathbf{4}$ & $\mathrm{CH}_{3}$ & $\mathrm{Bg}$ & $\mathrm{CH}_{3}$ \\
$\mathbf{5}$ & $\mathrm{CH}_{3}$ & $\mathrm{Bg}$ & $\mathrm{C}_{11} \mathrm{H}_{23}$ \\
$\mathbf{6}$ & $\mathrm{CH}_{3}$ & $\mathrm{Bg}$ & $\mathrm{PhOBz}$ \\
\hline $\mathbf{7}$ & $\mathrm{Bg}$ & $\mathrm{H}$ & $\mathrm{CH}_{3}$ \\
$\mathbf{8}$ & $\mathrm{Bg}$ & $\mathrm{H}$ & $\mathrm{C}_{11} \mathrm{H}_{23}$ \\
$\mathbf{9}$ & $\mathrm{Bg}$ & $\mathrm{H}$ & $\mathrm{PhOBz}$ \\
\hline
\end{tabular}


<smiles>CCOC(=O)c1cc(OCc2ccccc2)cc(OCc2ccccc2)c1</smiles>

(a) $\mathrm{BgH}$

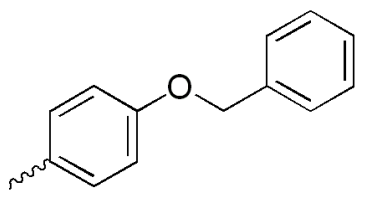

(b) $\mathrm{PhOBz}$

Figure 2.4 Bulky groups used in to modify the merocyanines 


\section{Chapter 3}

\section{UV-Vis Spectroscopic Studies}

\subsection{Concentration Dependant Absorption Spectra}

\subsubsection{Introduction}

UV-Vis absorption spectroscopy is a convenient way of observing aggregates as the absorption spectrum of an aggregate is different to that of the monomer. Two different geometries of aggregates, $\mathrm{H}$ - and J-aggregates, are associated with spectral shifts in opposite directions relative to the main absorption band of the monomer. These red and blue shifted absorption bands were first observed by Jelly and Scheibe in the 1930's when they undertook extensive studies of the UV-Vis absorption spectra of cyanine dyes. ${ }^{47,48}$ In these studies Jelly observed a narrow absorption band, red-shifted compared to the main absorption peak, and this was named the J-band (after Jelly ${ }^{39}$ ). Scheibe undertook UVVis studies on the solvent, concentration, and temperature dependence of the excitation behaviour of cyanine dyes and proposed that this peak was due to a loosely bound, “reversible polymerisation". ${ }^{49}$ This picture of aggregation was later supported through the work of Franck and Teller, ${ }^{50}$ and Kasha ${ }^{40}$, who applied Frenkel exciton theory ${ }^{51,52}$ to the data. The blue-shifted band also associated with aggregation of a head-to-tail geometry is named the H-band because of the resulting hypsochromic shift in absorption.

Aggregation can be observed by UV-Vis absorption spectroscopy because the molecules 


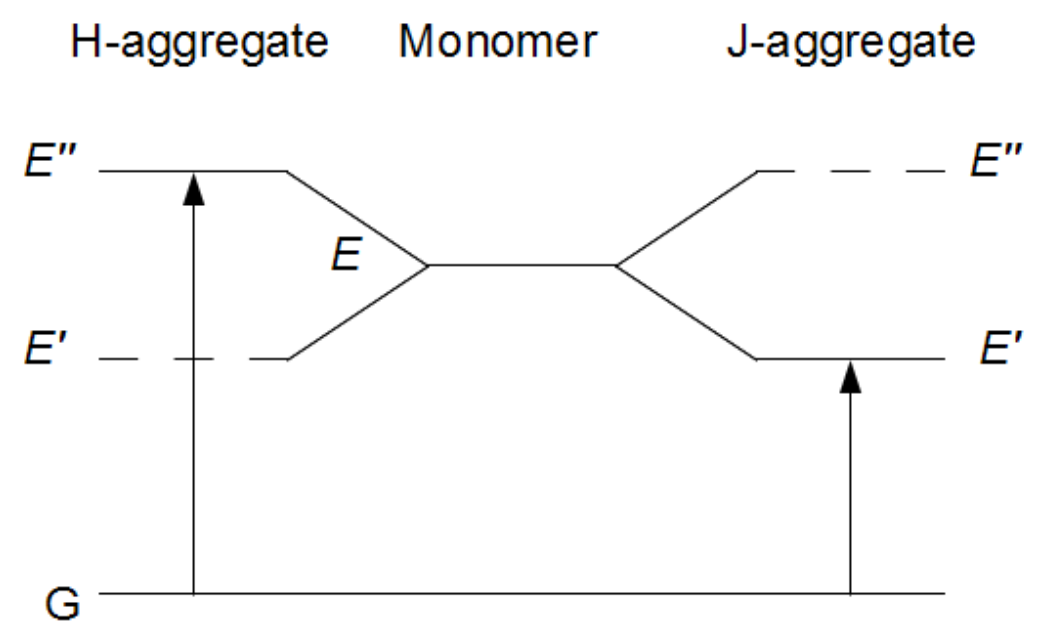

Figure 3.1 The allowed absorption states of aggregates compared to the monomer. The allowed transitions are shown with an arrow, forbidden states are dashed.

in an aggregate are closely associated and electronic coupling between them results in an exciton splitting of the absorption band from the absorption of a single molecule. The energy splitting that results is depicted in figure 3.1. In the case of $\mathrm{H}$-aggregates the parallel transition dipoles and the parallel dipole conformation result in repulsion, so increase the energy of the excited state $E^{\prime \prime}$, while the antiparallel case lowers the van der Waals interactions and the splitting to lower energy. ${ }^{53}$ However the $E^{\prime}$ transition is forbidden, so the allowed $E^{\prime \prime}$ transition results in the higher energy, blue-shifted, absorption. In J-aggregates the splitting to lower energy $E^{\prime}$ is allowed, while the antiparallel case $E^{\prime \prime}$ is forbidden. Hence the J-aggregate spectrum is red-shifted relative to that of the monomer.

The typical absorption spectrum of a solution where a chromophore exists in a monomeric form and as an aggregate consists of a main absorption band with a red- or blueshifted side band. To confirm that this side band is attributable to the formation of an aggregate, the absorption spectrum of the chromophore is measured over a range of concentrations. At higher concentrations aggregate formation is favoured over the 
monomer form. Thus a comparison the UV-Vis absorption spectra of the chromophore over a range of concentrations will show that an increase in the aggregation absorption band relative to the monomer band occurs as the concentration is increased.

Full experimental detail of the concentration dependant UV-Vis absorption studies is given in section 6 . The results of these aggregation experiments are given below (section 3.1.2).

\subsubsection{Concentration Dependant Absorption Results}

The polarity of the solvents used in the concentration study is important because the solvating environment has an effect on whether the chromophores will tend towards aggregate formation or favour their monomeric form in solution. To cover a range of polarities the concentration studies were performed in three solvents: 1,4-Dioxane ( $\varepsilon=2.30)$, chloroform $(\varepsilon=4.81)$, and dimethyl sulfoxide $(\varepsilon=46.7)$. In a low polarity solvent a chromophore with a zwitterionic ground state is not stabilised as much as it would be in a more polar solvent, and so of the solvents used in this study, 1,4-dioxane (lowest polarity) is the solvent where the largest amount of aggregation is expected to be seen.

Figures 3.2 - 3.12 show the concentration dependant absorption spectra of compounds 1

-9. The arrows on each figure indicate increasing concentration, and the spectrum of the lowest concentration sample is shown by a dashed line. 


\section{Concentration Effects on UV-Vis Absorption Spectra in 1,4-Dioxane}

Figures 3.2 to 3.10 present the concentration dependence of the absorption spectra of chromophores $\mathbf{1}$ - 9 in 1,4-dioxane. 1,4-Dioxane is the least polar solvent used in this study (see table 3.3), and was chosen because the majority of the chromophores studied are reasonably soluble in dioxane, unlike other low polarity solvents such as toluene, in which only a few chromophores are soluble. It should be noted that compound $\mathbf{1 0}$ with $\mathrm{R}_{1}=\mathrm{CH}_{3} ; \mathrm{R}_{2}=\mathrm{H} ; \mathrm{R}_{3}=\mathrm{CH}_{3}$ is insufficiently soluble to record absorption spectra over a wide range of concentrations and so the simplest member of the chromophore family included in this study is compound $\mathbf{1}$, which has a $\mathrm{C}_{10} \mathrm{H}_{21}$ alkyl chain in the $\mathrm{R}_{1}$ position to increase solubility in low polarity solvents.

The concentration dependence of the absorption spectrum of compound $\mathbf{1}$ in dioxane is shown in figure 3.2. The lowest concentration $\left(1.00 \times 10^{-6} \mathrm{~mol} . \mathrm{dm}^{-3}\right)$ is shown with the dashed line. This spectrum shows two absorption bands, a main band with a maximum at $632 \mathrm{~nm}$ and a shoulder at $547 \mathrm{~nm}$. The concentration of the chromophore in the dioxane solution was progressively increased to $2.73 \times 10^{-5}$ mol.dm ${ }^{-3}$. The change in the absorption spectra as the concentration was increased is indicated by the arrows (with the direction the arrow points corresponding to increasing concentration). As can be seen in the figure, increasing the concentration of the solution causes an increase in the minor band at $547 \mathrm{~nm}$ relative to the main band at $632 \mathrm{~nm}$. Because of this concentration dependant behaviour the absorption band at $632 \mathrm{~nm}$ is attributed to the monomer absorption and the $547 \mathrm{~nm}$ absorption band to absorption by an aggregate.

Figures 3.3 and 3.4 show the same study repeated with compounds $\mathbf{2}$ and $\mathbf{3}$ respectively. 
These compounds are similar to compound $\mathbf{1}$, but with $\mathrm{R}_{3}=\mathrm{C}_{11} \mathrm{H}_{23}$ in compound $\mathbf{2}$ and $\mathrm{R}_{3}=\mathrm{PhOBz}$ in compound $\mathbf{3}$.

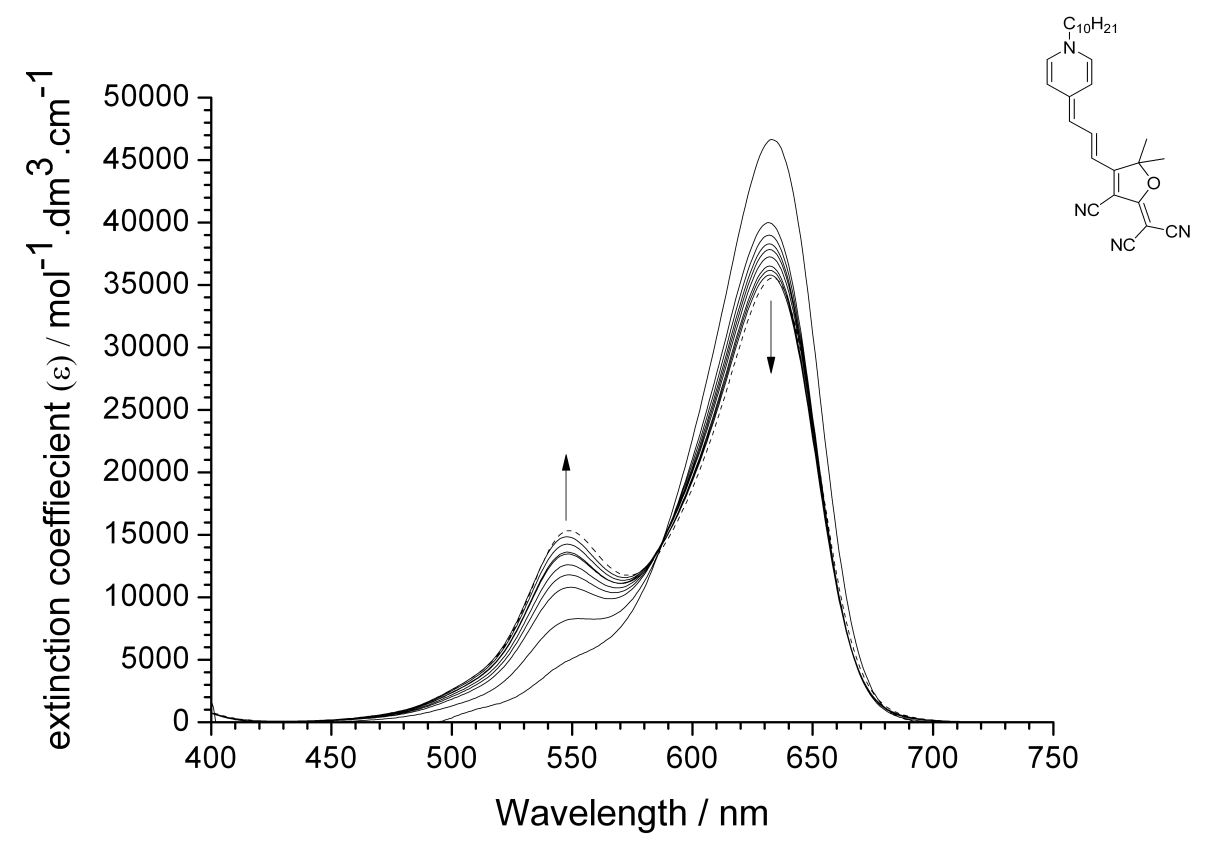

Figure 3.2 Concentration dependence of the UV-Vis absorption spectrum of compound 1 in 1,4-dioxane. The structure of $\mathbf{1}$ is shown in the top right. The arrows indicate changes upon increasing concentration. 
Compounds $\mathbf{1}$ - $\mathbf{3}$ show significant aggregation with increasing concentration. This is expected in compound $\mathbf{1}$ as it has the least bulky side groups. The bulky groups added to the $\mathrm{R}_{3}$ site were designed to create an increased steric hindrance to aggregation by adding bulky groups around the acceptor end of the molecule. In compound $\mathbf{3}$, the addition of the bulky groups containing phenyl rings was expected to have an effect on the aggregation behaviour of the compounds because of intermolecular interactions between the $\pi$ electrons associated with the bulky groups in adjacent molecules.

A bulky group was added to the $\mathrm{R}_{2}$ position to increase the steric hindrance through the central part of the molecule. Figure 3.5 shows the absorption spectra of compound 4 , where $\mathrm{R}_{1}=\mathrm{R}_{3}=\mathrm{CH}_{3}$ and $\mathrm{R}_{2}=\mathrm{Bg}$. Figures 3.6 and 3.7 show compounds $\mathbf{5}$, where $\mathrm{R}_{1}=\mathrm{CH}_{3}, \mathrm{R}_{2}=\mathrm{Bg}, \mathrm{R}_{3}=\mathrm{C}_{11} \mathrm{H}_{23}$; and $\mathbf{6}$ where $\mathrm{R}_{1}=\mathrm{CH}_{3}, \mathrm{R}_{2}=\mathrm{Bg}$, and $\mathrm{R}_{3}=\mathrm{PhOBz}$. Note that while compound $\mathbf{1 1}\left(\mathrm{R}_{1}=\mathrm{R}_{3}=\mathrm{CH}_{3}\right.$ and $\left.\mathrm{R}_{2}=\mathrm{H}\right)$ is not soluble in dioxane, addition of the bulky group to the $\mathrm{R}_{2}$ position increases the solubility and so compounds 4 - 6, with $\mathrm{R}_{1}=\mathrm{CH}_{3}$ are sufficiently soluble to be studied.

A bulky group was also substituted at the $R_{1}$ position. These compounds $\mathbf{7}$ to 9 are shown in figures 3.8 and 3.10. Note that these compounds have limited solubilities in dioxane.

\section{Concentration Effect on UV-Vis Absorption Spectra in Chloroform}

Figure 3.11 shows the absorption spectra of compound 1 in chloroform. Chloroform has a dielectric constant of 4.81 , i.e. it is more polar than 1,4-dioxane which has a $\varepsilon=2.30$. The absorption maxima of the main absorption band is somewhat blue shifted compared to the absorption $\lambda_{\max }$ in dioxane. This trend is repeated for all of compounds 2-9 as well 


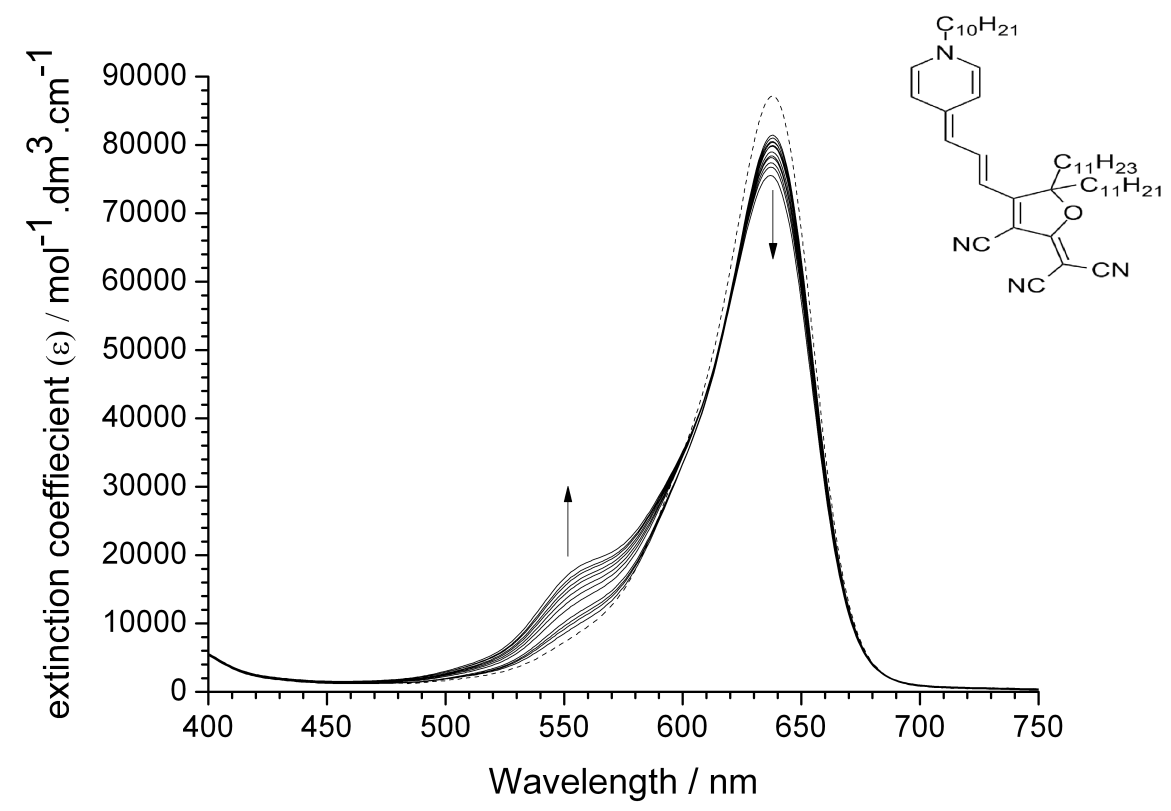

Figure 3.3 Concentration dependence of the UV-Vis absorption spectrum of compound 2 in 1,4-dioxane. The structure of $\mathbf{2}$ is shown in the top right. The arrows indicate changes upon increasing concentration.

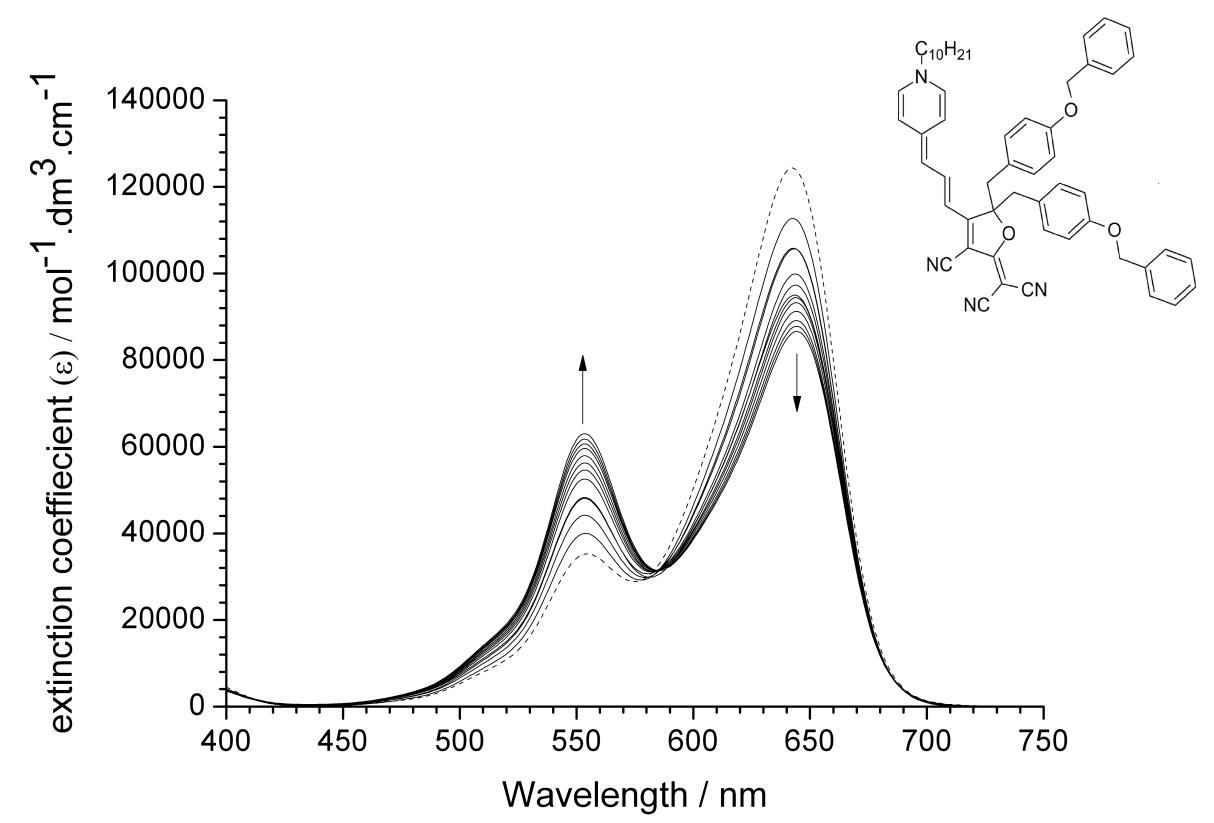

Figure 3.4 Concentration dependence of the UV-Vis absorption spectrum of compound 3 in 1,4-dioxane. The structure of $\mathbf{3}$ is shown in the top right. The arrows indicate changes upon increasing concentration. 


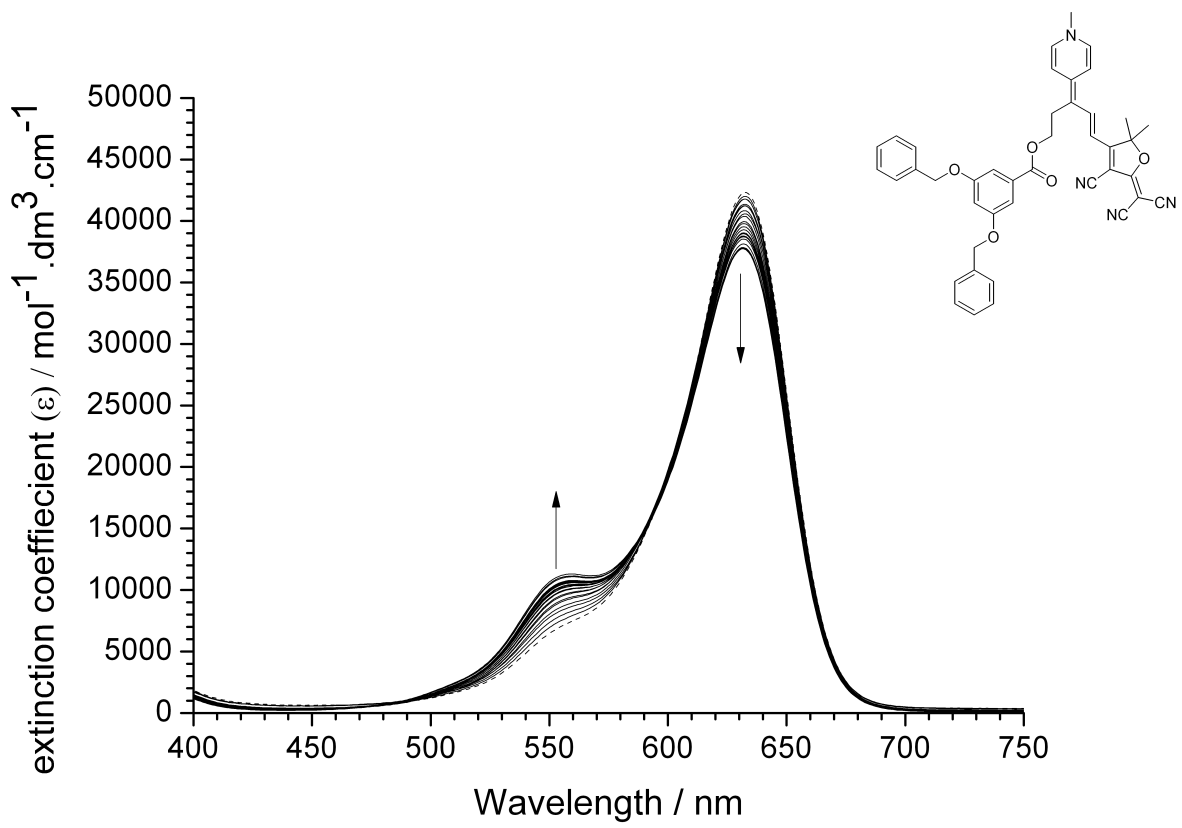

Figure 3.5 Concentration dependence of the UV-Vis absorption spectrum of compound 4 in 1,4-dioxane. The structure of $\mathbf{4}$ is shown in the top right. The arrows indicate changes upon increasing concentration.

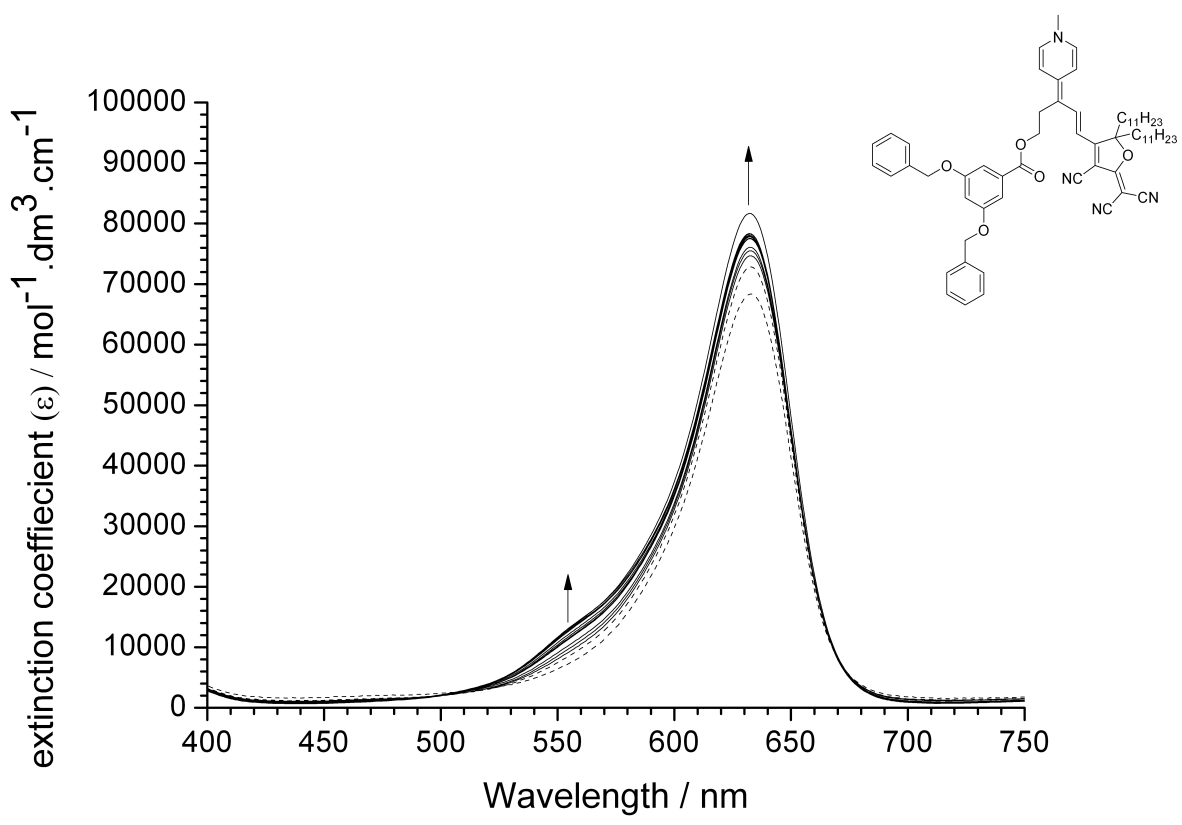

Figure 3.6 Concentration dependence of the UV-Vis absorption spectrum of compound 5 in 1,4-dioxane. The structure of $\mathbf{5}$ is shown in the top right. The arrows indicate changes upon increasing concentration. 


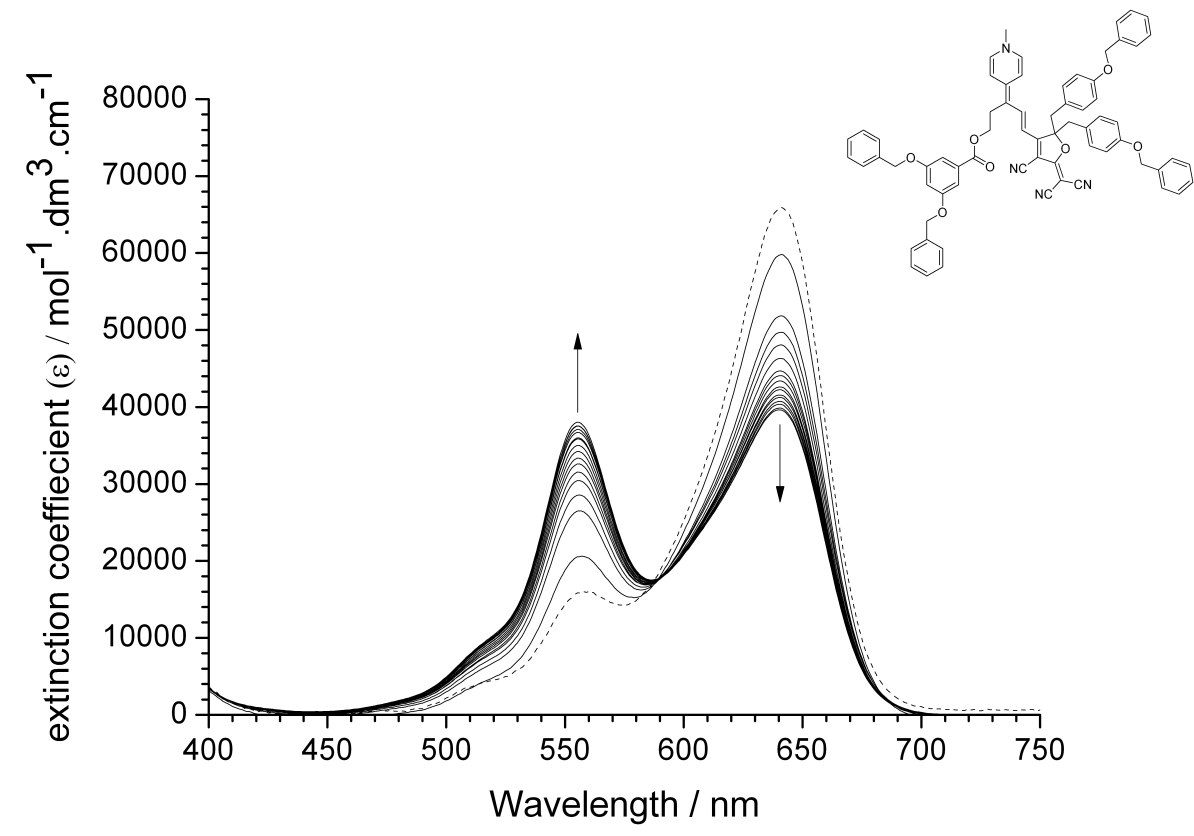

Figure 3.7 Concentration dependence of the UV-Vis absorption spectrum of compound 6 in 1,4-dioxane. The structure of $\mathbf{6}$ is shown in the top right. The arrows indicate changes upon increasing concentration.

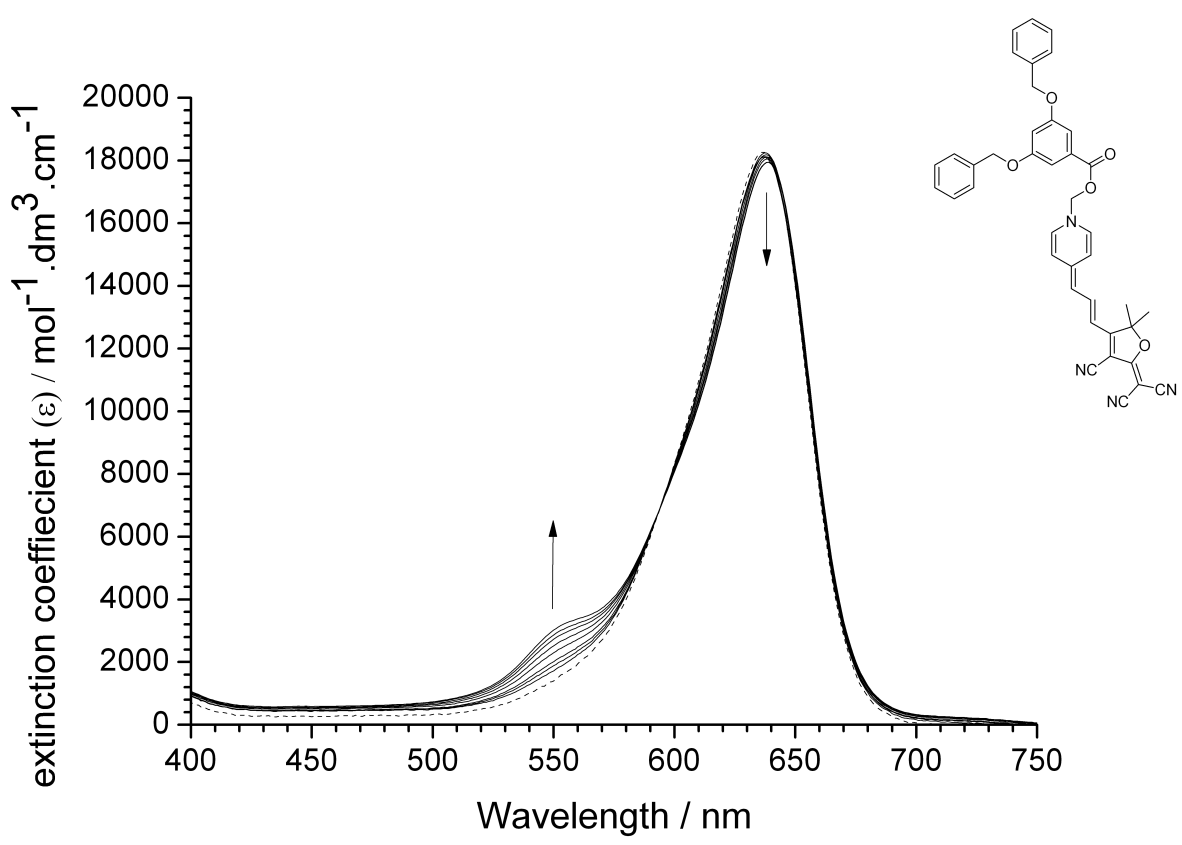

Figure 3.8 Concentration dependence of the UV-Vis absorption spectrum of compound 7 in 1,4-dioxane. The structure of $\mathbf{7}$ is shown in the top right. The arrows indicate changes upon increasing concentration. 


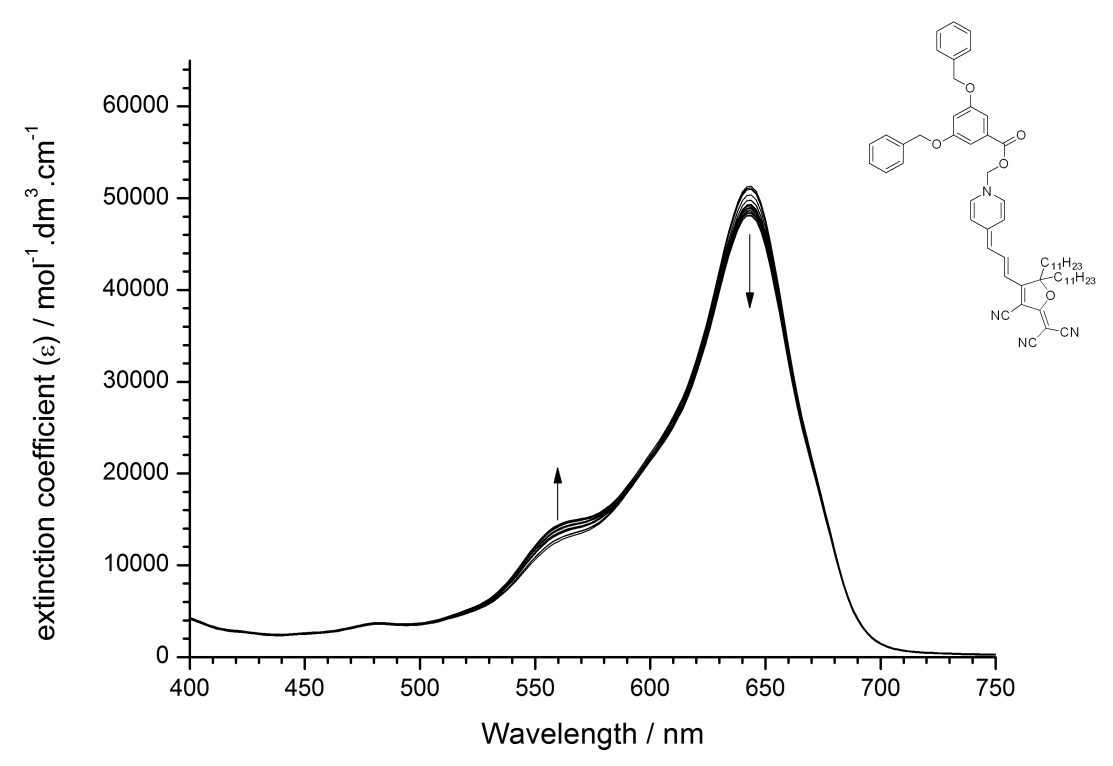

Figure 3.9 Concentration dependence of the UV-Vis absorption spectrum of compound $\mathbf{8}$ in 1,4-dioxane. The structure of $\mathbf{8}$ is shown in the top right. The arrows indicate changes upon increasing concentration.

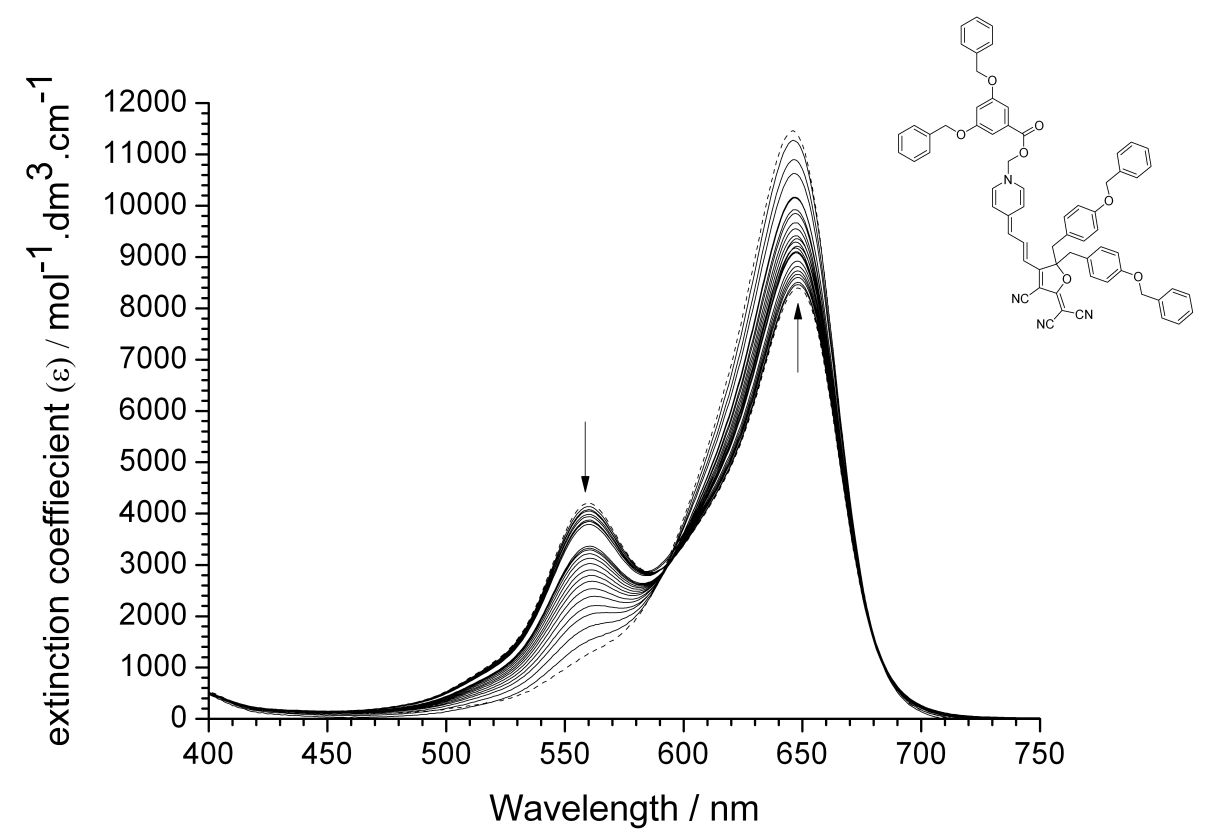

Figure 3.10 Concentration dependence of the UV-Vis absorption spectrum of compound 9 in 1,4-dioxane. The structure of 9 is shown in the top right. The arrows indicate changes upon increasing concentration. 
as can be seen in summary table 3.1. Such a hypsochromic shift is typically exhibited by zwitterionic merocyanines and is consistent with previous reports. ${ }^{1,27,54}$

As the merocyanines are highly dipolar molecules it is expected that solvents with high polarities such as chloroform will have a stabilising effect on the monomer in solution, and thus less aggregation should occur. The chloroform absorption spectra do not show a concentration dependant shoulder band as was present in 1,4-dioxane which suggests that in chloroform an aggregation does not occur.

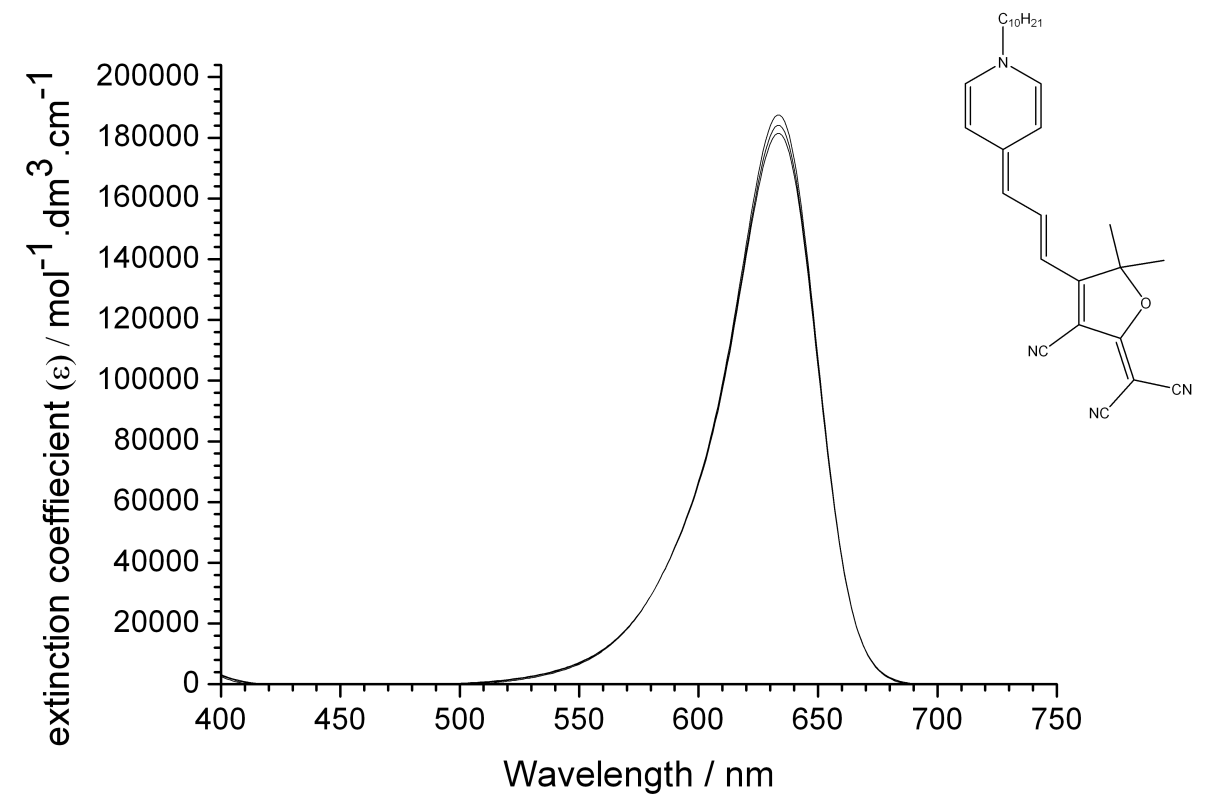

Figure 3.11 Concentration dependence of the UV-Vis absorption spectrum of compound $\mathbf{1}$ in chloroform. The structure of $\mathbf{1}$ is shown in the top right.

\section{Concentration Effect on UV-Vis Spectra in Dimethylsulfoxide}

The experiments were also repeated in dimethyl sulfoxide and these results are shown in figures 3.12 and additional figures in the appendix. DMSO has a high dielectric constant $(\epsilon=46.7)$ and is the most polar solvent used in this study. Figure 3.12 shows an absorption 
spectrum with only one absorption band, as was the case in chloroform. As DMSO is a more polar solvent than chloroform, aggregation is not expected to occur, and the presence of a single peak confirms that it does not.

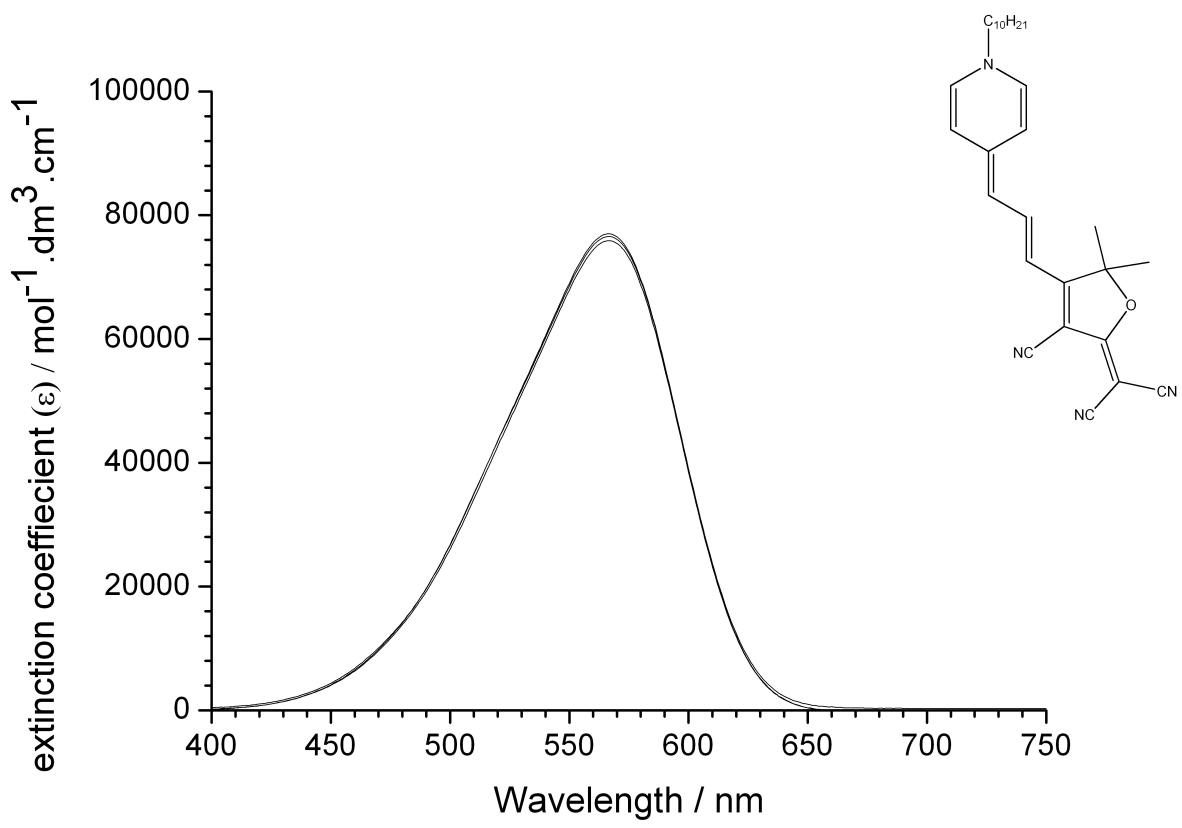

Figure 3.12 Concentration dependence of the UV-Vis absorption spectrum of compound $\mathbf{1}$ in DMSO. The structure of $\mathbf{1}$ is shown in the top right.

\section{Dimerization constants}

From the absorption spectra shown in figures 3.2 to 3.10 the dimerization constants of compounds 1 to 3 in dioxane were determined. As a dimer forms, the initial concentration of the chromophore in solution $\left(c_{0}\right)$ can be separated into two parts, the concentration of the monomeric dye $\left(c_{M}\right)$ and a dimer $\left(c_{D}\right)$. The fraction of the monomeric form of the dye, $(\alpha)$, in solution is therefore given by equation 3.1 .

$$
\alpha=\frac{c_{M}}{c_{0}}
$$




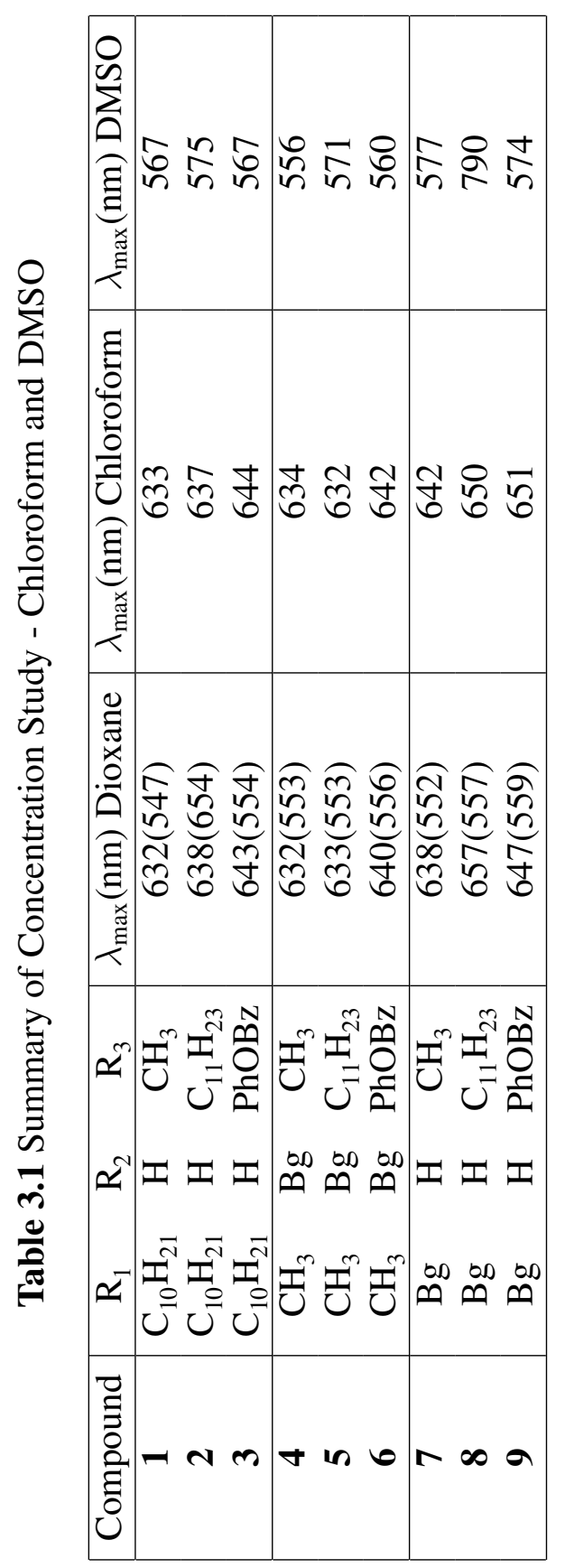


Table 3.2 Values of of $\mathrm{K}_{d i m}$

\begin{tabular}{|c|c|c|c|}
\hline Compound & $K_{\text {dim }}\left(\mathrm{dm}^{3} \mathrm{~mol}^{-1}\right)$ & Standard Error & R-square \\
$\mathbf{1}$ & 11416 & 501 & 0.92 \\
$\mathbf{2}$ & 3260 & 252 & 0.80 \\
$\mathbf{3}$ & 25803 & 1568 & 0.89 \\
\hline
\end{tabular}

The apparent extinction coefficient $(\varepsilon)$ of the measured spectra is the sum of the contributions from the monomeric and dimer forms of the chromophore and can be represented by equation 3.2 .

$$
\varepsilon=\varepsilon_{M} \alpha+(1-\alpha) \varepsilon_{D}
$$

The dimerization constant $K_{d i m}$ can be calculated by assuming a simple $2 \mathrm{M} \rightleftharpoons \mathrm{D}$ equilibrium of dimer and monomer in solution giving equation 3.3.

$$
K_{\operatorname{dim}}=\frac{c_{D}}{c_{M}^{2}}
$$

Equations 3.2 and 3.3 can be combined to give 3.4

$$
\varepsilon=\frac{\sqrt{8 K_{\operatorname{dim}} c_{0}+1}-1}{4 K_{\operatorname{dim}} c_{0}}\left(\varepsilon_{M}-\varepsilon_{D}\right)+\varepsilon_{D}
$$

Using this equation to fit plots of $\varepsilon$ against concentration allows the value of $K_{d i m}$ to be calculated. The summary of these $\mathrm{K}_{d i m}$ values is given in table 3.2. 


\subsection{Solvatochromism}

\subsubsection{Introduction}

Solvatochromism is the term given to the change in the position of the absorption maximum of a compound in solution caused by changing the polarity of the solvating environment. This occurs due to differences in the solvation energies of the ground and excited states as the medium is changed. This is illustrated in figure 3.13, which shows an important mechanism contributing to solvatochromism in a schematic form. Because the excited state is usually more polar than the ground state, more polar solvents favour its stabilisation compared to the ground state, which results in a smaller energy gap as polarity increases, causing a bathochromic (red) shift in the absorption energy. If the ground state is more polar than the excited state it is preferentially stabilised, leading to a hypsochromic (blue) shift. ${ }^{55}$

The usual definition of solvatochromism holds that an increase in the polarity of the medium leads to a bathochromic shift in the absorption maximum ${ }^{56}$ and this is typically observed in neutral ground state dyes such as disperse red 1. However in zwitterionic ground state dyes the opposite holds and an increase in the solvent polarity leads to an hypsochromic shift in the absorption maximum - a phenomenon known as negative solvatochromism. ${ }^{57}$ 


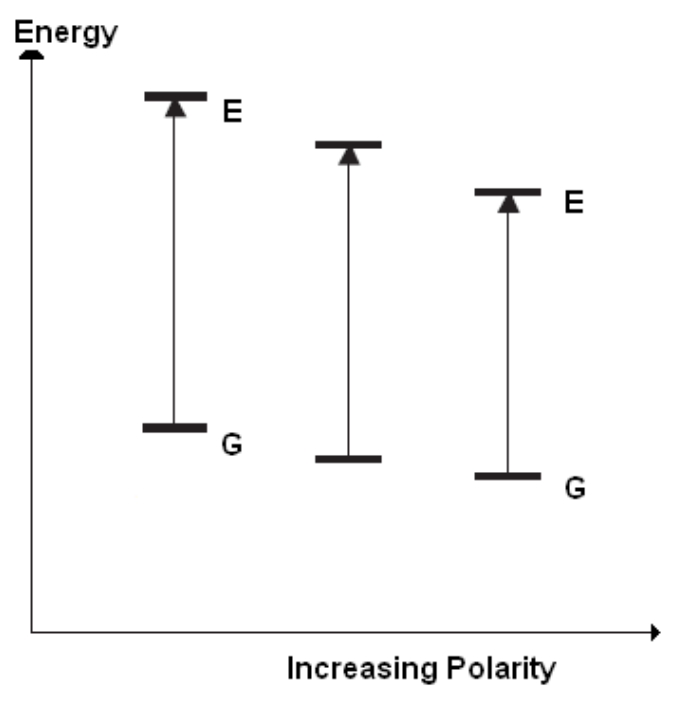

Figure 3.13 Simplified electronic state energy level diagram showing a bathochromic shift in absorption energy. As the solvent polarity is increased the excited state experiences a greater stabilization than the ground state, and so the energy gap decreases. This results in a red shift of the absorption spectrum.

The effect that the solvent has on the energies of the excited and ground states of the chromophores used in this study is important, because the solvent-solute interactions and solvating environment have a significant effect on the behaviour of the chromophores in solution and this can affect aggregation, as well as isolated chromophore-chromophore interactions. In highly polar solvents the RHS merocyanines, which have high dipole moments in their ground states, are stabilised in highly polar environments. This favours the monomeric forms. In a non-polar solvent, the solvent molecules surrounding the dipolar merocyanine monomer do not have this stabilising effect and aggregate formation will be favoured. ${ }^{57,58}$

To study the effect of these solvent-solute interactions on the formation of aggregates a range of solvents has been investigated. The choice of solvents was made to cover as wide a range of polarities as possible, however, this choice of solvents has been limited by the 
solubility of the chromophores, which are insoluble in many solvents such as water and ethanol.

The solvents used in this solvatochromism study and their dielectric/solvating properties are given in table 3.3. Two measures of solvent polarity are given in this table, dielectric constant $(\epsilon)$ and $\mathrm{E}_{\mathrm{T}}(30)$. "Solvent Polarity" is the overall solvation ability of the solvent. The process of the dissolution of a solute in a solvent depends on all interactions between both solute and solvent molecules, including specific interactions, such as Columbic interactions, hydrogen-bonding, and Van der Waals forces. Quantifying solvent polarity must include the effects of these specific forces, which are different for each solvent and solute. Considering the solvent as an idealised homogeneous continuum, physical constants such as the solvent's dielectric constant (also known as relative permittivity) give a partial measure of solvent polarity/stabilisation. The dielectric constant is determined by measuring the strength of an electric field between two charged plates when they are in a vacuum $\left(E_{0}\right)$ and the when the space is filled by solvent. The ratio $E_{0} / E$ gives a numerical value for the relative permittivity $\left(\varepsilon_{r}\right)$. Where specific solvent-solvent interactions are important it can be more appropriate to use empirical solvent polarity scales. These scales are based on solvent-dependant chemical reactions or spectral absorptions. ${ }^{59}$ The $\mathrm{E}_{\mathrm{T}}(30)$ solvent scale is such an empirical measurement based on the shift in the absorption maximum of a dye, pyridinium N-phenolate betaine, in various solvents. This scale was used because of the large number of solvents for which data is known, providing a more comprehensive range of options for solvent choice than other similar empirical scales. 
Table 3.3 Properties of solvents used in this study, as reported by Wohlfarth ${ }^{60}$ and by Reichardt ${ }^{56}$.

\begin{tabular}{|c|c|c|c|}
\hline Solvent & Molecular Formula & Dielectric constant $(\varepsilon)^{60}$ & $\mathrm{E}_{\mathrm{T}}(30)^{56} / \mathrm{kcal} \cdot \mathrm{mol}^{-1}$ \\
\hline toluene & $\mathrm{C}_{6} \mathrm{H}_{5} \mathrm{CH}_{3}$ & 2.38 & 33.9 \\
benzene & $\mathrm{C}_{6} \mathrm{H}_{6}$ & 2.30 & 34.3 \\
1,4-dioxane & $\mathrm{C}_{4} \mathrm{H}_{8} \mathrm{O}_{2}$ & 2.30 & 36.0 \\
chlorobenzene & $\mathrm{C}_{6} \mathrm{H}_{5} \mathrm{Cl}$ & 5.65 & 6.8 \\
tetrahydrofuran & $\mathrm{C}_{4} \mathrm{H}_{8} \mathrm{O}$ & 7.47 & 37.4 \\
chloroform & $\mathrm{CHCl}_{3}$ & 4.81 & 39.1 \\
cyclohexanone & $\left(\mathrm{CH}_{2}\right)_{5} \mathrm{CO}$ & 15.7 & 39.4 \\
1,1,2-trichloroethane & $\mathrm{C}_{2} \mathrm{H}_{3} \mathrm{Cl}$ & 7.2 & 40.3 \\
pyridine & $\mathrm{C}_{5} \mathrm{H}_{5} \mathrm{~N}$ & 13.55 & 40.5 \\
dichloromethane & $\mathrm{CH}_{2} \mathrm{Cl}$ & 40.7 \\
acetone & $\left(\mathrm{CH}_{3}\right)_{2} \mathrm{CO}$ & 21.4 & 42.2 \\
dimethyl formamide & $\left.\left(\mathrm{CH}_{3}\right)_{2} \mathrm{NC}_{2} \mathrm{O}\right) \mathrm{H}$ & 38 & 43.8 \\
dimethyl sulfoxide & $\left(\mathrm{CH}_{3}\right)_{2} \mathrm{SO}$ & 46.7 & 45.1 \\
acetonitrile & $\mathrm{CH}_{3} \mathrm{CN}$ & 37.5 & 45.6 \\
methanol & $\mathrm{CH}_{3} \mathrm{OH}$ & 33.5 & 55.4 \\
\hline
\end{tabular}

In addition to comparing behaviour in individual solvents, binary solvent mixtures were used in order to separate the effects of specific solvent-solute interactions from the effect of solvent polarity. The absorption spectra of a series of mixed-solvent solutions were measured, varying the mole fraction of two solvents in the mixture from 0 to 1 .

\subsubsection{Results}

\section{Solvatochromism}

The maximum absorption and emission energies of compounds $\mathbf{1}$ - 9 are shown in figure 3.14, which uses the solvent's dielectric constant, $\varepsilon$, as a measure of the solvent polarity, while figure 3.15 uses the $\mathrm{E}_{\mathrm{T}}(30)$ scale. The figures also include emission data which will be discussed in the next section.

The merocyanines show a hypsochromic (blue) shift in the wavelength of the absorption 
Table 3.4 Dielectric constants of mixed solvent systems as reported by Wohlfarth ${ }^{60}$

\begin{tabular}{|c|c|c|c|}
\hline \multicolumn{2}{|c|}{ Acetonitrile-1,4-dioxane } & \multicolumn{2}{|c|}{ Acetonitrile-chloroform } \\
\hline Mole Fraction of Acetonitrile & Dielectric Constant & Mole Fraction & Dielectric Constant \\
\hline 0 & 2.21 & 0 & 4.8065 \\
\hline 0.078 & 3.35 & 0.1037 & 7.6076 \\
\hline 0.153 & 4.88 & 0.2537 & 11.842 \\
\hline 0.288 & 8.03 & 0.2963 & 13.068 \\
\hline 0.41 & 11.27 & 0.3998 & 16.114 \\
\hline 0.52 & 14.55 & 0.4983 & 19.142 \\
\hline 0.619 & 18.01 & 0.6023 & 22.451 \\
\hline 0.709 & 21.39 & 0.6957 & 25.604 \\
\hline 0.791 & 24.85 & 0.7984 & 29.431 \\
\hline 0.903 & 30.52 & 0.8986 & 33.249 \\
\hline 0.946 & 32.97 & 1 & 37.4 \\
\hline 1 & 36.01 & - & - \\
\hline
\end{tabular}

maxima with increasing solvent polarity. This solvatochromic behaviour can be explained in terms of the resonance forms the merocyanine ground state that can exist, ranging from the neutral molecule to the fully zwitterionic form. In medium polarity solvents such as THF, the absorption spectrum shows a narrow absorption band suggesting that the ground state has contributions from both the neutral and zwitterionic states. As solvent polarity increases, the increase in the energy gap and the broadening of the absorption band indicate a shift in the ground state to a more polyene-like state.

\section{Binary Solvent Mixtures}

To identify specific solvent interactions, binary solvent mixtures were used. Figure 3.16 shows the absorption spectra of $\mathbf{6}$ in a series of acetonitrile-chloroform mixtures. These start with a mole fraction of 0 in chloroform and $\lambda_{\max }=578 \mathrm{~nm}$. As the mole fraction of chloroform was increased the absorption spectra became successively red shifted, approaching the $\lambda_{\max }$ of $\mathbf{6}$ in pure chloroform $(642 \mathrm{~nm})$. This shift in $\lambda_{\max }$ is also shown 


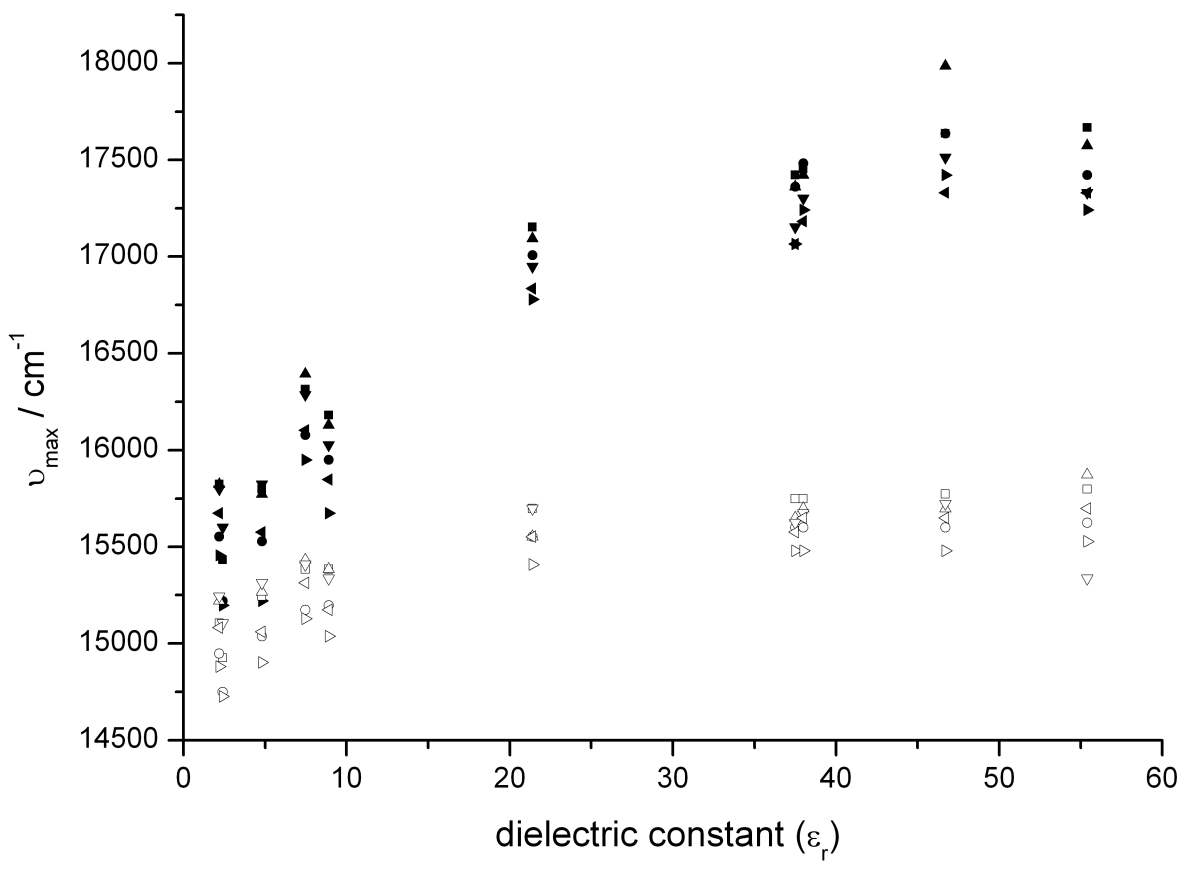

Figure 3.14 Graph showing (a) the absorption maximum in reciprocal wavelength (solid symbols) and (b) the emission maximum (open symbols) against the dielectric constant of the solvent. Key: compound $1(\sqcup), \mathbf{3}(\diamond), \mathbf{4}(\triangle), \mathbf{5}(\nabla), \mathbf{1 2}(\triangleleft), \mathbf{9}(\triangleright)$

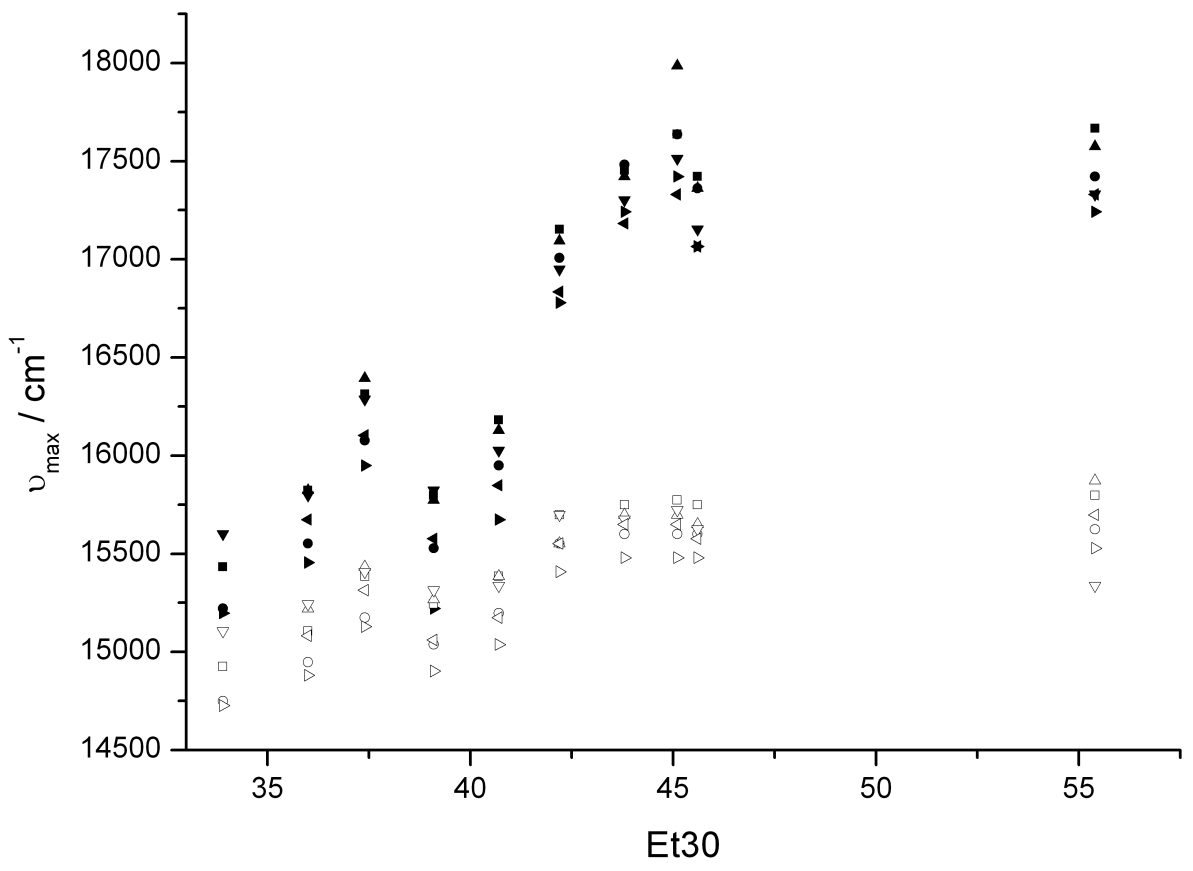

Figure 3.15 Graph showing (a) the absorption maximum in reciprocal wavelength (solid symbols) and (b) the emission maximum (open symbols) against the solvent polarity $\left(\mathrm{E}_{\mathrm{T}}(30)\right)$. Key: compound $1(\sqcup), \mathbf{3}(\diamond), \mathbf{4}(\triangle), \mathbf{5}(\nabla), \mathbf{1 2}(\triangleleft), \mathbf{9}(\triangleright)$ 
in figure 3.17 .

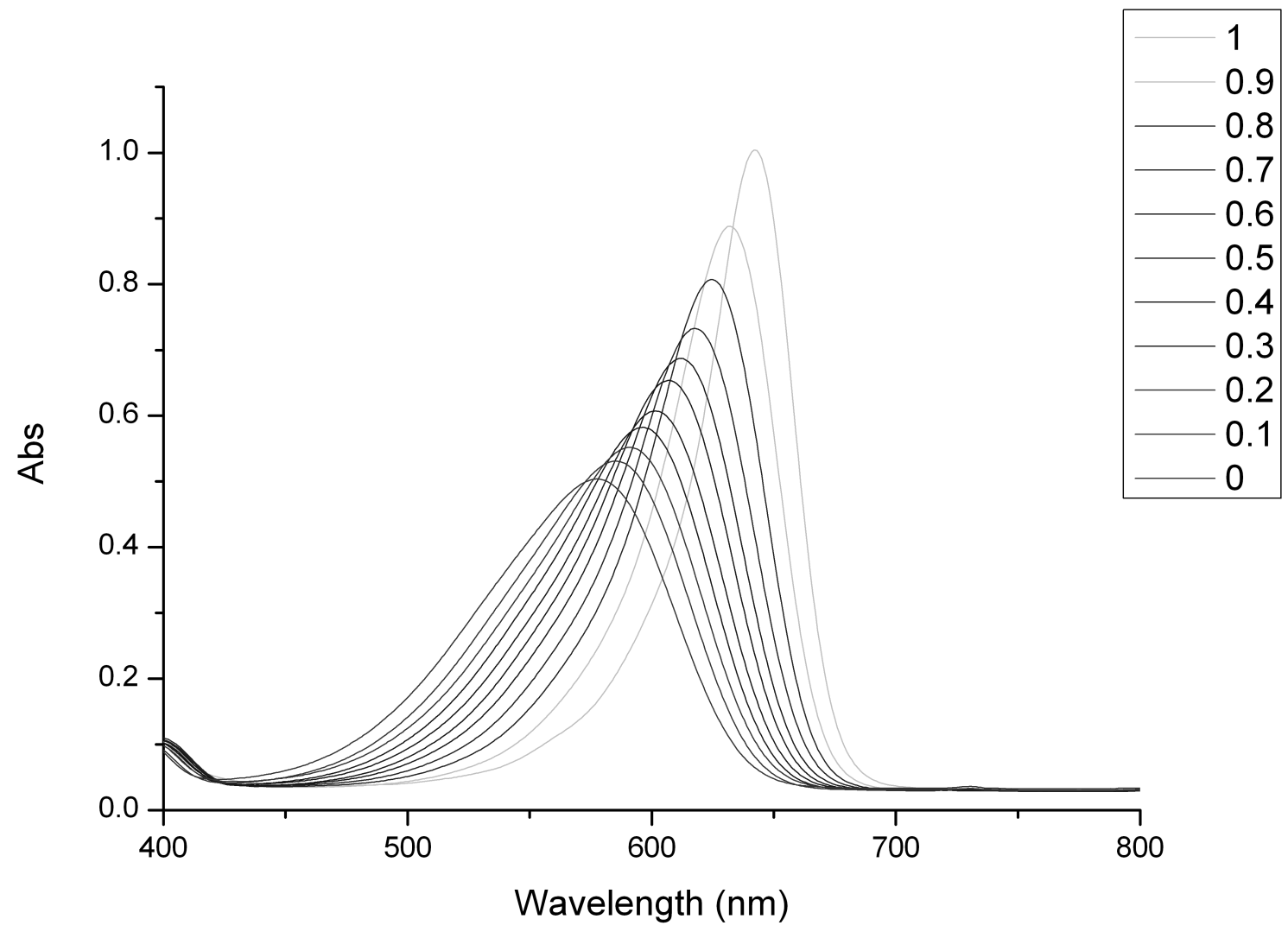

Figure 3.16 Absorption spectra of compound 6 for the different acetonitrile-chloroform solvent mole fractions. 


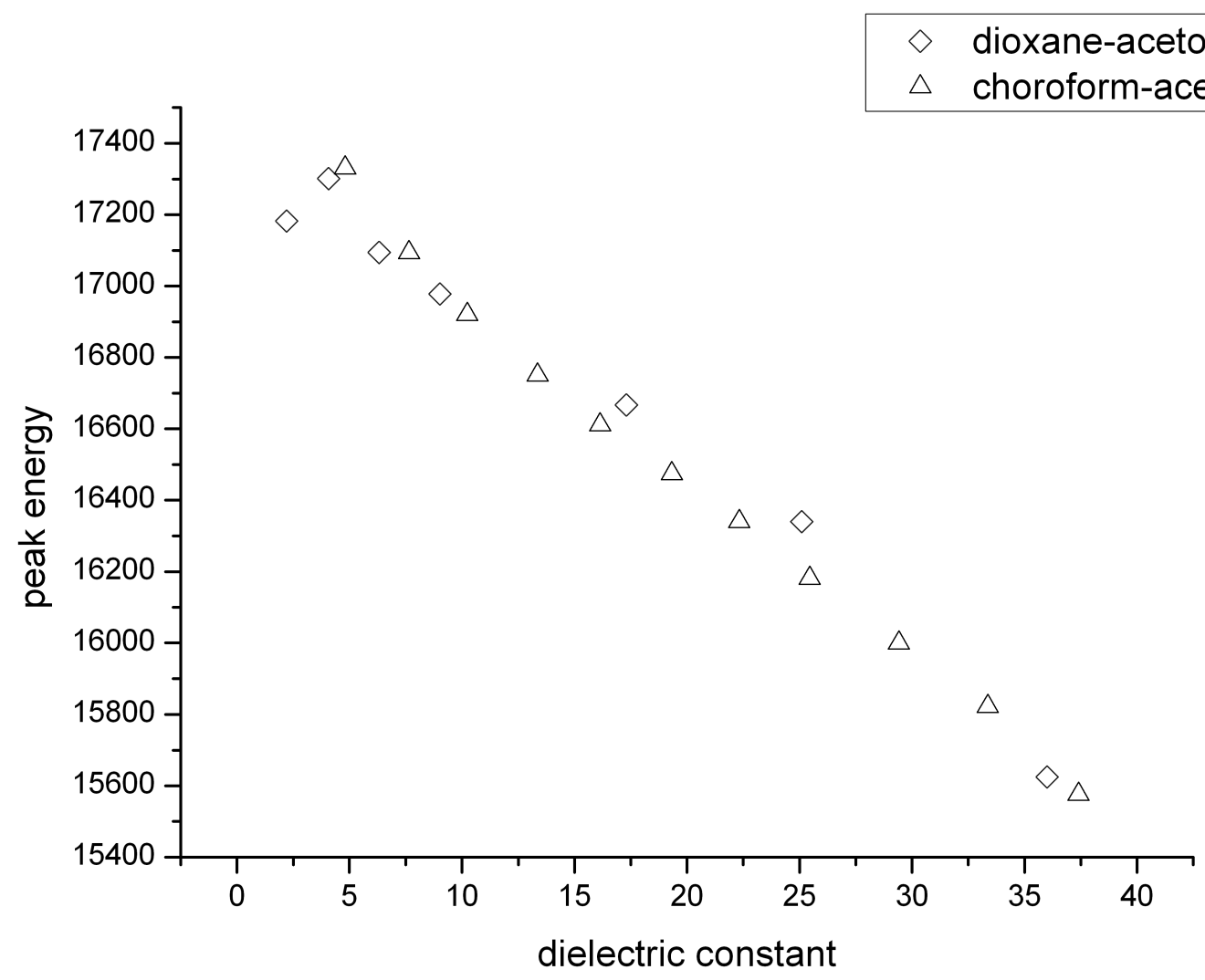

Figure 3.17 Absorption maxima of compound 6 plotted against dielectric constant.

\subsection{Fluorescence}

\subsubsection{Introduction}

The transitions associated with the excitation of an electron from the highest energy occupied orbital of a molecule in its electronic ground state to an excited state by absorbing a photon of UV radiation or visible light is shown in figure 3.18. Fluorescence is the converse of this process, where an electron in the lowest energy orbital of an excited state relaxes to the ground state energy level, releasing the excitation energy, as a photon of light. Additionally the excitation energy can be lost non-radiatively as 
transfer of vibrational energy to the solvent. Absorption of a photon and transfer of the electron to the higher energy state takes place within a femtosecond $\left(10^{-15} \mathrm{~s}\right)$ but the much heavier atomic nuclei within the molecule can not change their positions or momenta within this time. ${ }^{61}$ Thus, the nuclear configuration directly after absorption is identical to the ground state nuclear configuration immediately before absorption of a photon, a phenomenon known as the Franck-Condon principle. ${ }^{62,63}$ As a result, absorption occurs into an excited vibrational state within the excited electronic level. Through non-radiative internal conversion the excited electron rapidly decays via internal and intramolecular vibrational interactions to the lowest vibrational level $S_{1}$ and so the energy of the fluorescence emission is typically less than the absorption energy. This red-shift of the fluorescence spectrum compared to the absorption spectrum is known as the Stokes shift. ${ }^{64}$

Factors such as the Franck-Condon principle and the stokes shift mean that the fluorescence spectrum of a sample is not identical to its absorption spectrum. Fluorescence does not occur instantaneously, as soon as the absorption as occurred. The electron instead can spend time in the excited state and so has several possible paths to loosing the absorbed energy. Just as the electron is likely to decay from a higher vibrational level to the lowest vibrational level of the electronically excited state via collisions of the excited molecule with surrounding solvent molecules non-radiatively, non-radiatively transitions to the ground state can also occur, a process known as internal conversion (IC). Inter-system crossing (ISC) can also occur, where the electron in the first excited $S_{1}$ state can move to an iso-energetic vibrational level of the first excited triplet state $T_{1}$. From this triplet state the electron can again return to the ground state through IC or 


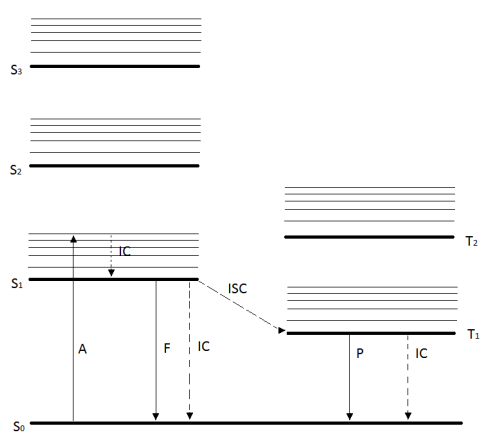

Figure 3.18 Jablonski diagram showing basic absorption and fluorescence transitions. Where $S_{0}$ is the ground state, $S_{1}, S_{2}, S_{3}$ are the excited singlet states, $T_{1}$ and $T_{2}$ are the excited triplet states. The arrow A represents the absorption of a photon; F, fluorescence of a photon; P, phosphorescence; IC, internal conversion; ISC, intersystem crossing.

phosphorescence. These radiative and non-radiative transitions are shown in figure 3.18.

Fluorescence lifetime measurements are used to give a measure of the kinetics and probability of a molecule in its excited state returning to its ground state with the emission of a photon or through non-radiative relaxation. These measurements are made by exposing the sample to a short pulse of light and recording the photons emitted by the sample over time, usually a period from picoseconds to nanoseconds. Fluorescence lifetime measurements can be made with a pulsed laser using a technique known as 'timecorrelated single-photon counting'. Modern photomultiplier tubes are sensitive enough to detect a very few photons and by using a repetitively pulsed laser source it is possible to record the intervals of time between the excitation pulses and detection of the emitted photons. In this way it is possible to build up the fluorescence decay profile, with a significantly improved signal-to-noise ratio than is the case in a system using only a single excitation pulse.

The fluorescence quantum yield gives a measure of the number of emitted photons relative to the number of absorbed photons as described in equation 3.5. The rate constants of 
both the emitted $(\Gamma)$ and non-radiative decay $\left(k_{n r}\right)$ both contribute to depopulating the excited state. Measurement of the ratio of $\Gamma$ to $\left(\Gamma+k_{n r}\right)$ gives the ratio of the photons emitted to the photons absorbed, the quantum yield, $\Phi$. This is close to unity if the nonradiative decay is much less than that of the radiative decay, i.e. nearly all photons that are absorbed are emitted as fluorescence. The experimentally determined fluorescence decay rate constant, $k_{\text {exp }}$, is the combination $\left(\Gamma+k_{n r}\right)$ and hence separation of $\Gamma$ from $k_{n r}$ is possible using equations 3.6 and 3.7.

$$
\begin{gathered}
\Phi=\frac{\Gamma}{\Gamma+k_{n r}} \\
\Gamma=\Phi k_{e x p} \\
k_{n r}=k_{\exp }(1-\Phi)
\end{gathered}
$$

Fluorescence lifetimes were measured for compounds $\mathbf{1}$ and $\mathbf{2}$ in dioxane and DMSO. In dioxane, lifetimes were measured using two different excitation wavelengths that excite primarily either into the monomer band $(630 \mathrm{~nm})$ or into the molecular dimer/aggregate band $(540 \mathrm{~nm})$ respectively. In DMSO, concentration studies discussed in section 3.1 did not show any evidence of molecular aggregation. Fluorescence lifetimes of the merocyanines in DMSO were measured using an excitation wavelength at $570 \mathrm{~nm}$ where the monomer absorbs and also at wavelengths to either side of the main peak (540 and 
$600 \mathrm{~nm}$ ) in order to determine if the fluorescence decay was dependent on the excitation wavelength.

\subsubsection{Results}

\section{Steady-State Fluorescence}

A selection of fluorescence spectra is shown for compound $\mathbf{1}$ in a number of solvents in figures 3.22 to 3.19 . Figures 3.22 to 3.19 show the fluorescence behaviour of compounds 1 to 9. The fluorescence emission spectrum in each case was recorded using excitation at the wavelength given in each figure. In solutions where no evidence of aggregates is apparent, (i.e. the absorption spectrum shows only a single peak), the fluorescence shows a typical "mirror-image" spectral shape and the excitation spectrum is similar to the absorption spectrum.

\section{Steady-State Fluorescence Spectra in 1,4,-dioxane}

The absorption spectra of compounds $\mathbf{1}$ to 9 in 1,4-dioxane all showed a minor absorption band at shorter wavelengths than the major absorption band. Excitation into both peaks results in fluorescence emission with the same spectral distribution, although excitation into the minor band results in fluorescence with a much lower intensity. The excitation spectra shown in figures 3.23 and 3.28 show a spectral distribution with only one peak, corresponding to the main absorption band and no evidence of a minor band associated with dimer/aggregate absorption. 


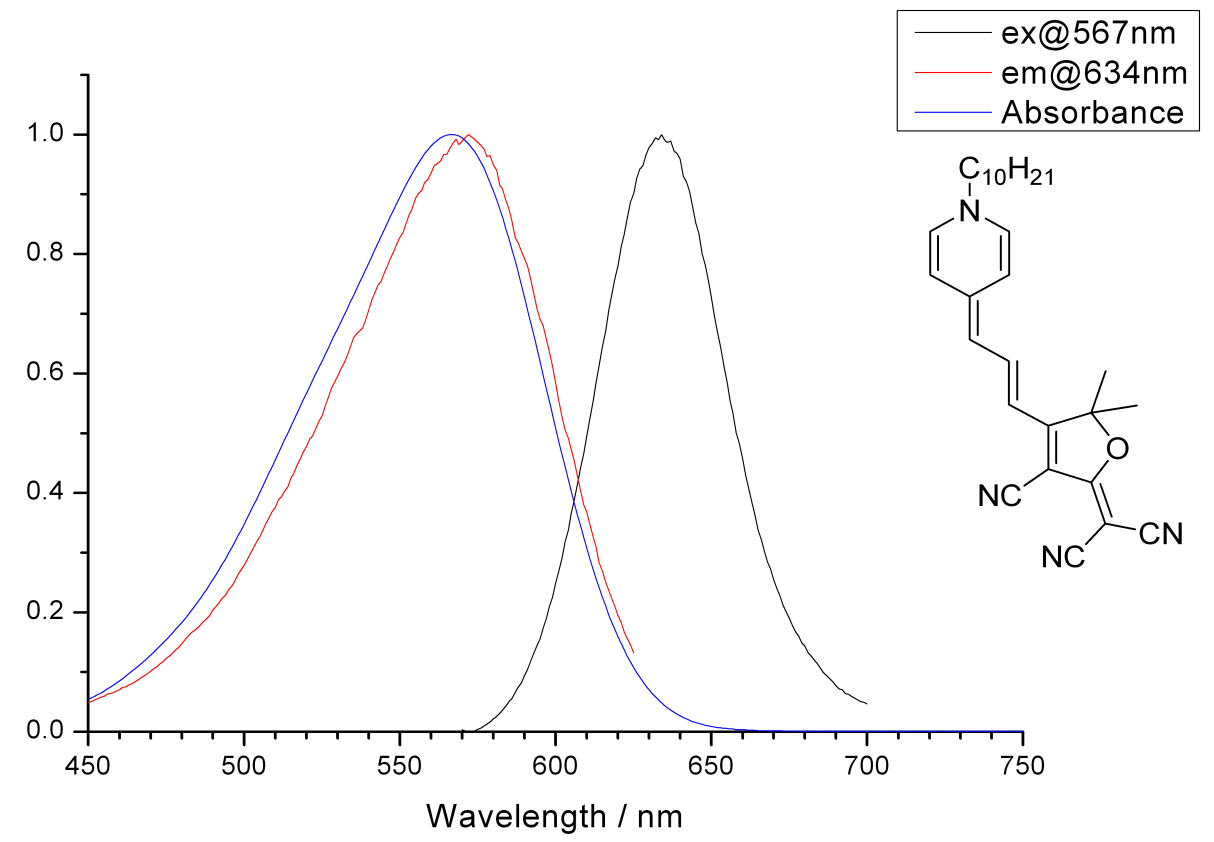

Figure 3.19 Normalised fluorescence emission and excitation spectra of compound $\mathbf{1}$ in DMSO

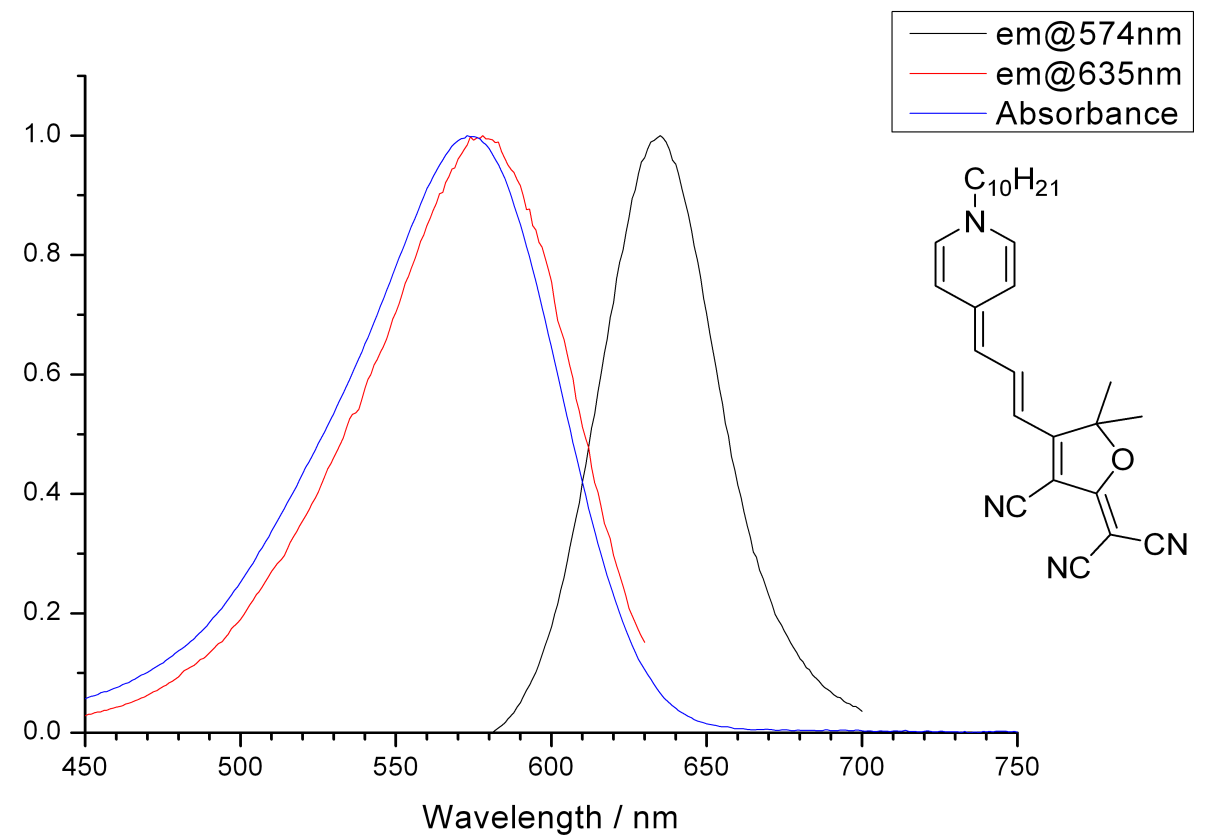

Figure 3.20 Normalised fluorescence emission and excitation spectra of compound 1 in acetonitrile 


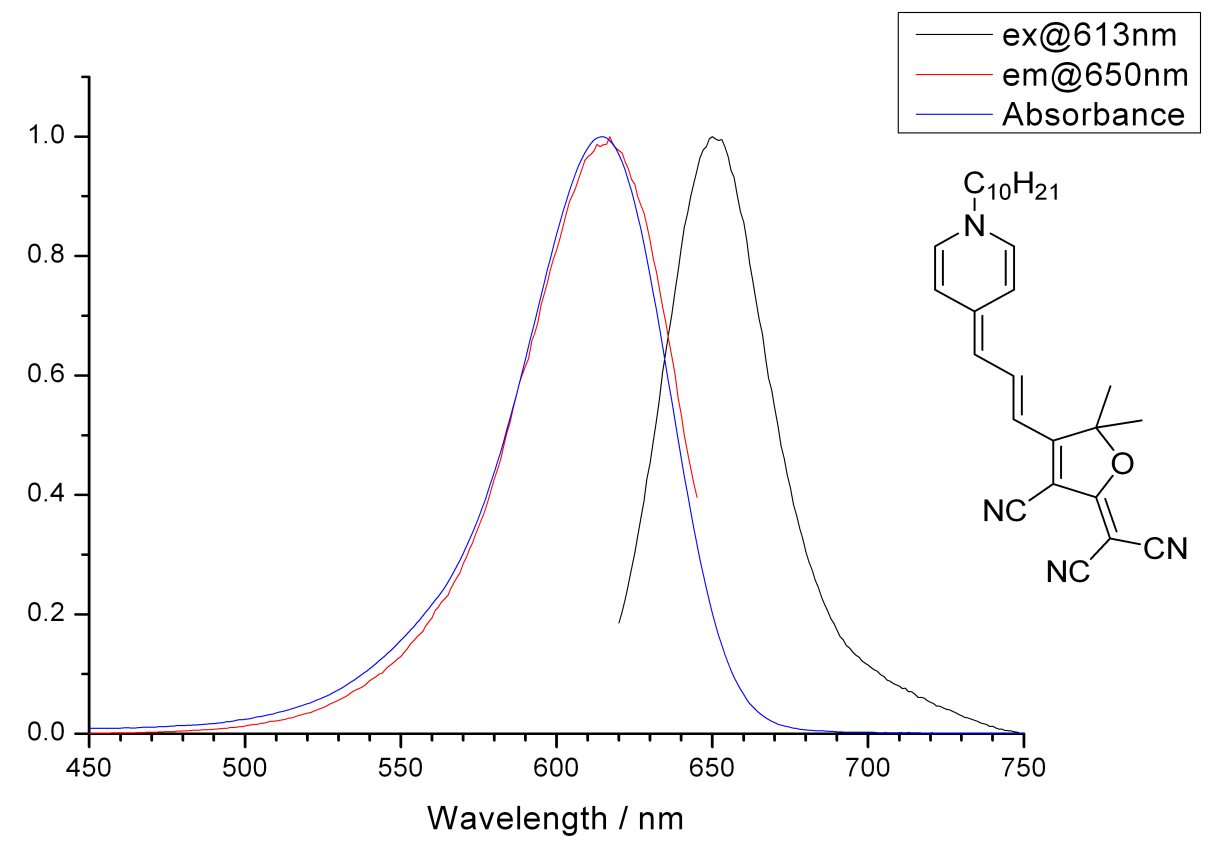

Figure 3.21 Normalised fluorescence emission and excitation spectra of compound $\mathbf{1}$ in THF

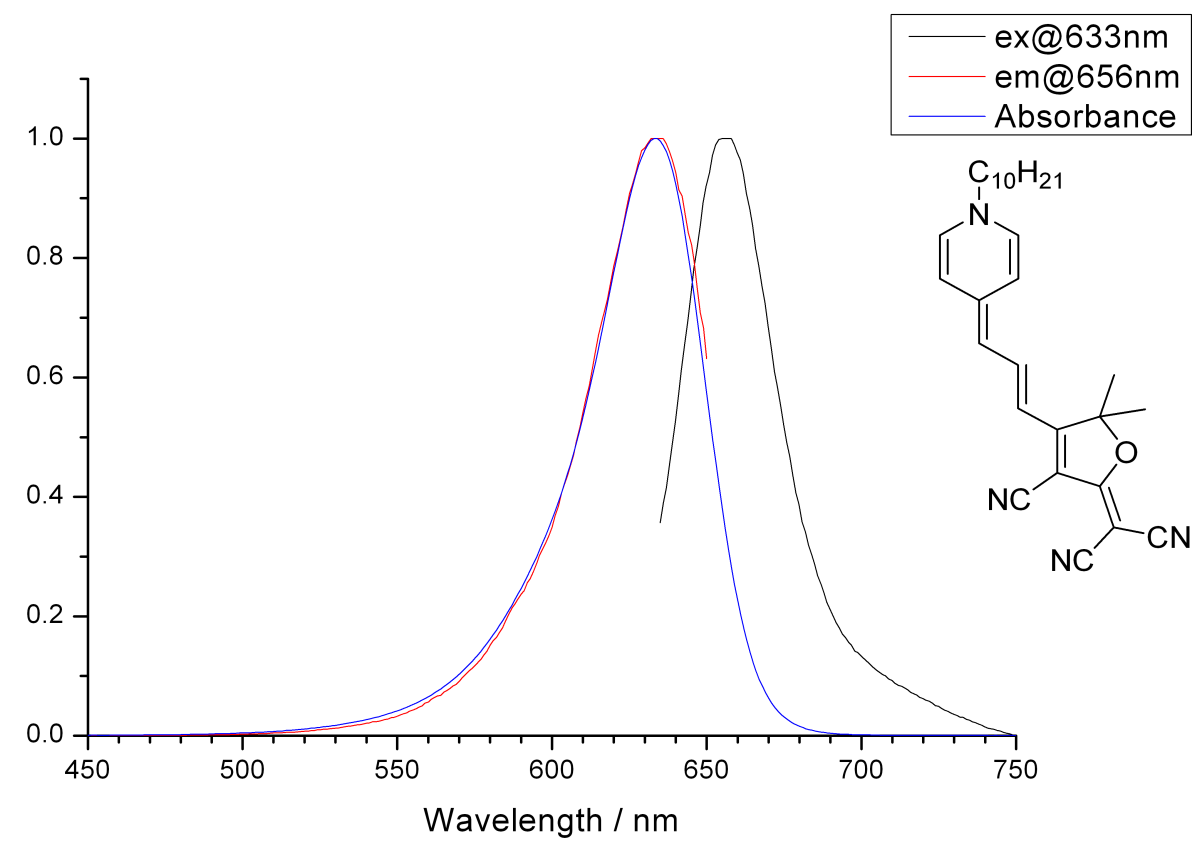

Figure 3.22 Normalised fluorescence emission and excitation spectra of compound $\mathbf{1}$ in chloroform 


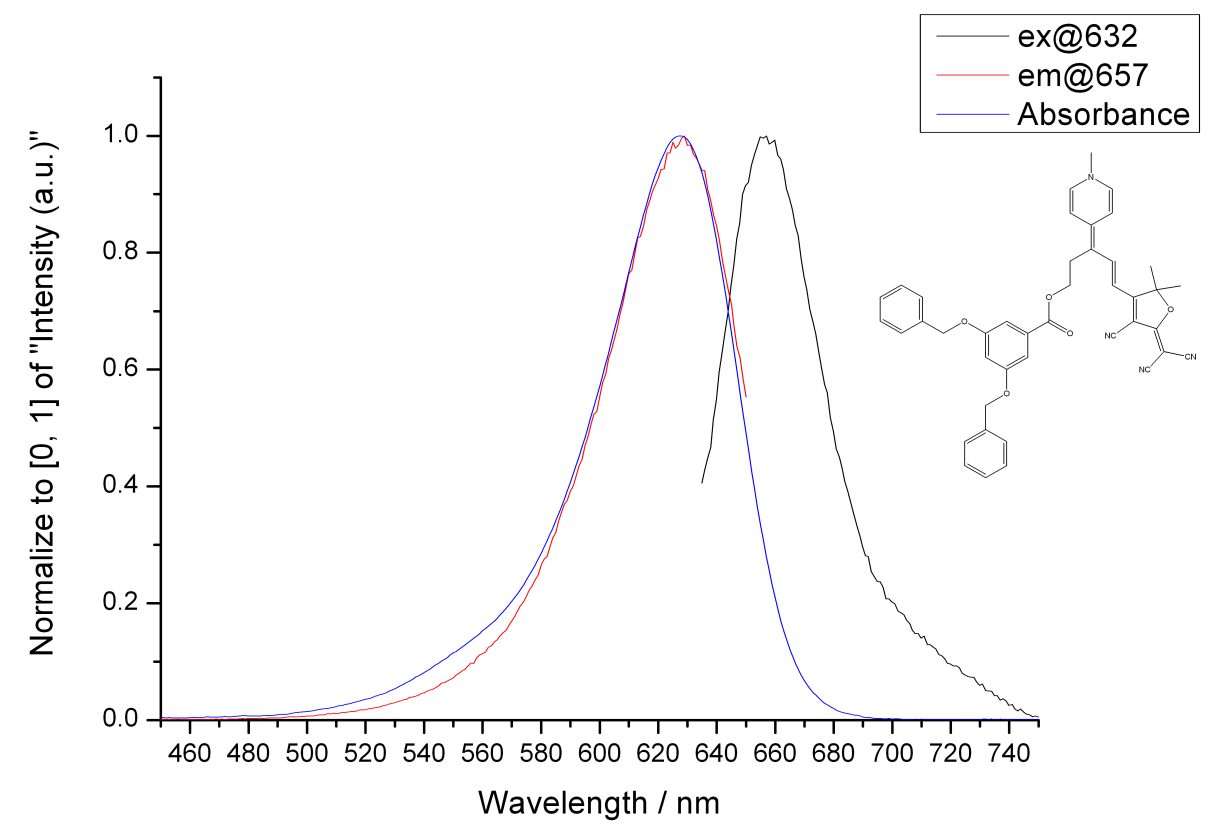

Figure 3.23 Normalised fluorescence emission and excitation spectra of compound 4 in dioxane

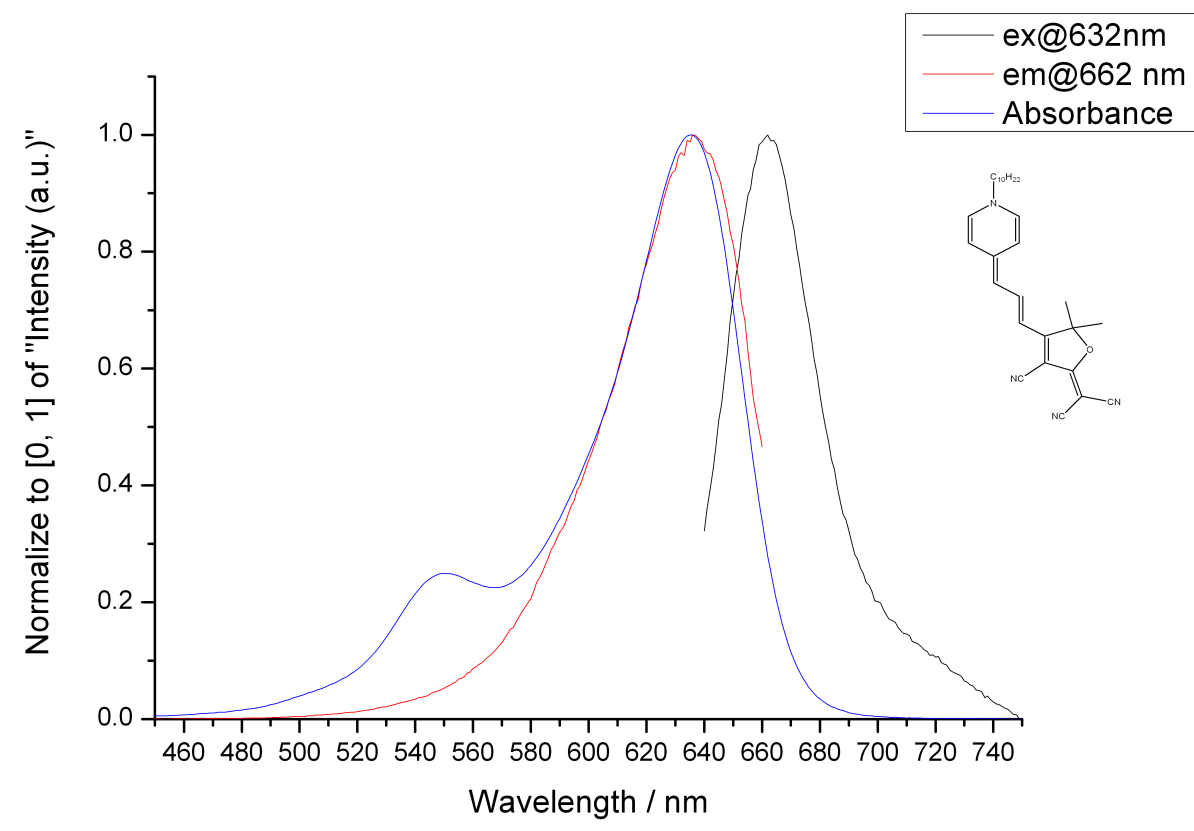

Figure 3.24 Normalised fluorescence emission and excitation spectra of compound $\mathbf{1}$ in dioxane 


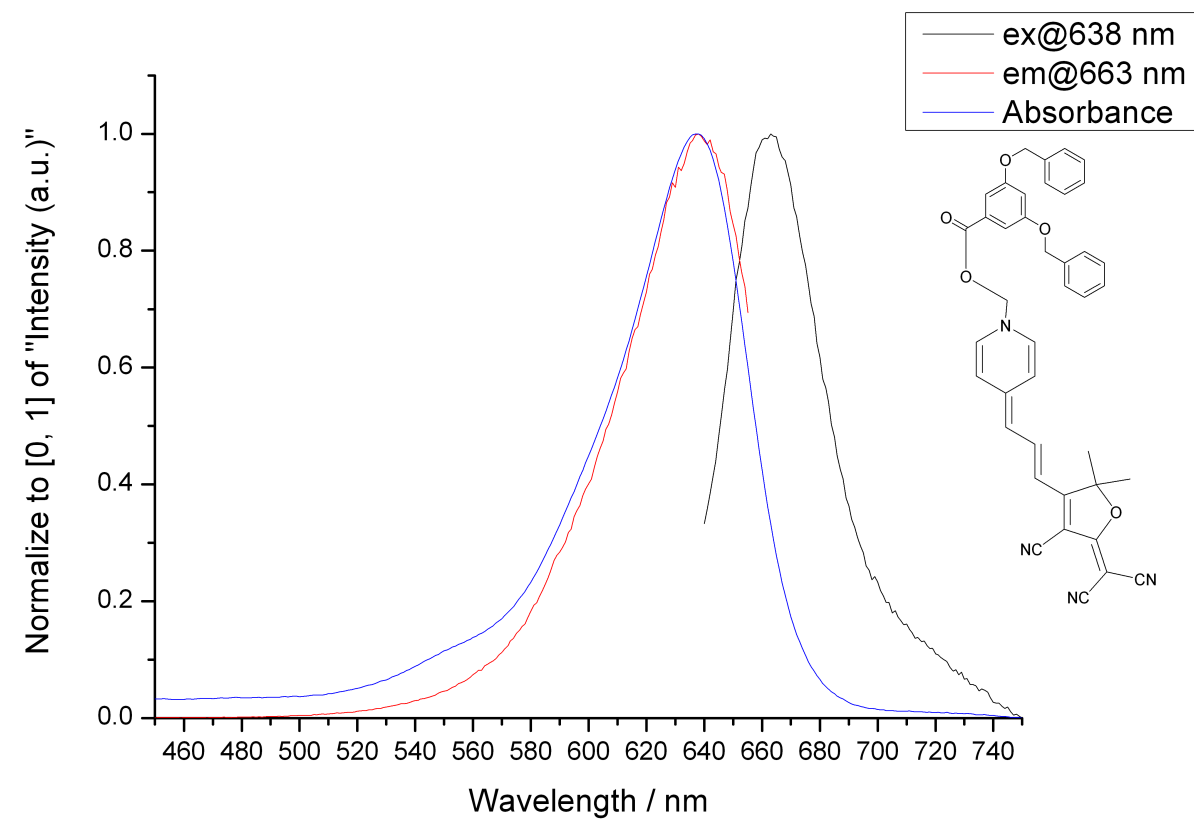

Figure 3.25 Normalised fluorescence emission and excitation spectra of compound $\mathbf{1 2}$ in dioxane

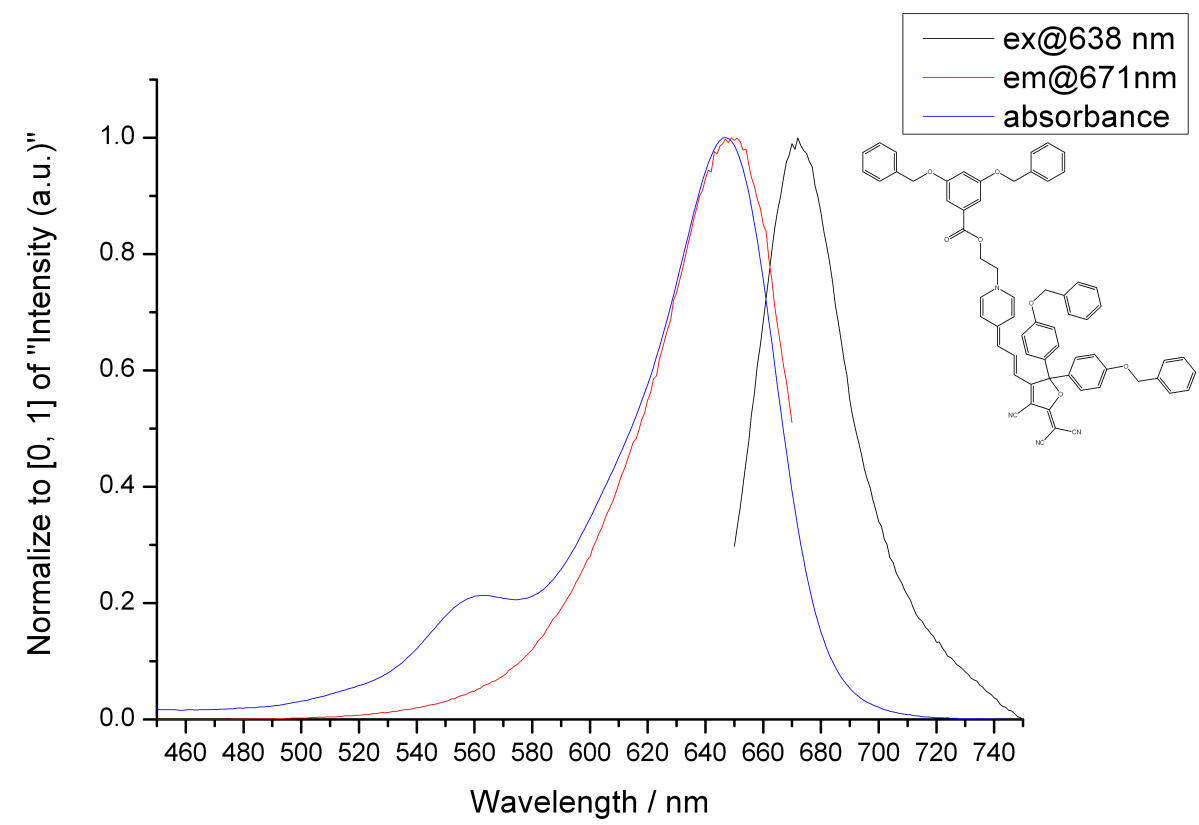

Figure 3.26 Normalised fluorescence emission and excitation spectra of compound 9 in dioxane 


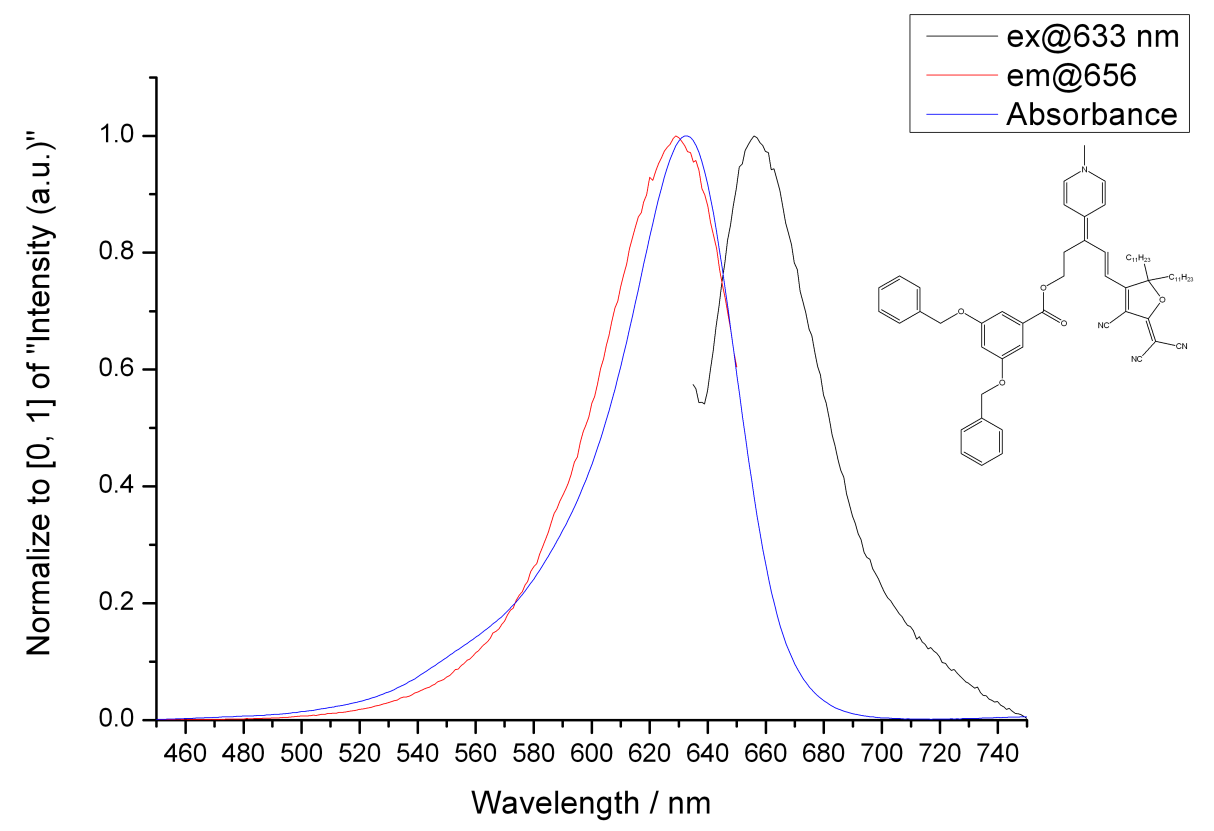

Figure 3.27 Normalised fluorescence emission and excitation spectra of compound $\mathbf{5}$ in dioxane

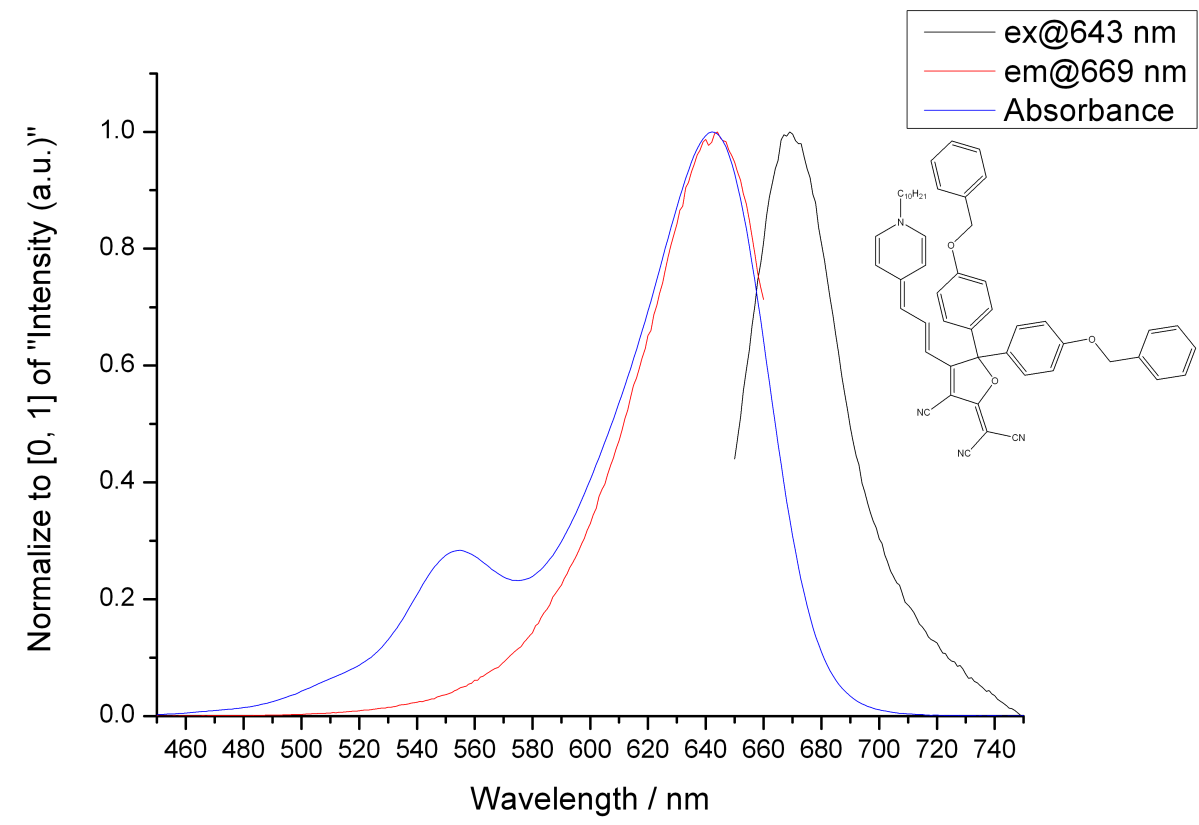

Figure 3.28 Normalised fluorescence emission and excitation spectra of compound $\mathbf{3}$ in dioxane 


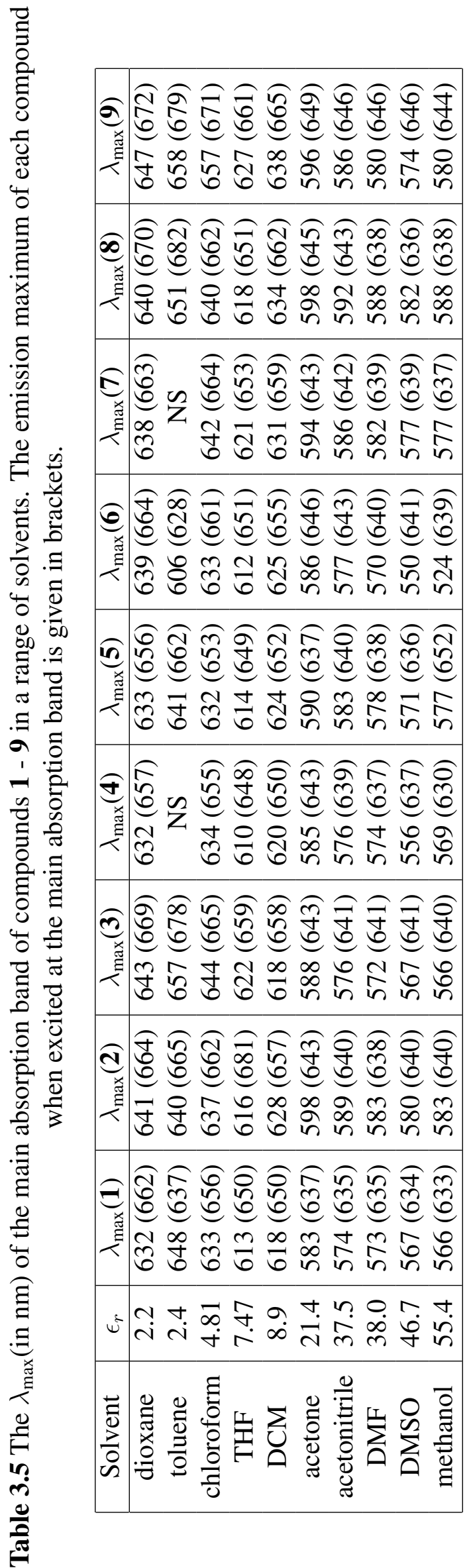




\section{Fluorescence Kinetics}

A summary of the fluorescence lifetime results is given in table 3.6. This table gives the excitation wavelengths used for each compound studied in the two solvents, dioxane and DMSO, and the decay lifetimes $\tau$ and pre-exponential factors $A$ determined from the decay curves by fitting to a sum of exponentials equation. Note that when the preexponential parameter is negative this exponential lifetime refers to a fluorescence rise time. To make it clear which $\tau$ values are rise times and which are decay times, these have been separated into two columns in the table.

\section{Fluorescence Quantum Yields}

Summaries of the fluorescence quantum yields for compounds $\mathbf{1}$ and $\mathbf{6}$ are given in table 3.7. The fluorescence emission of these chromophores lies in the spectral region of 630 - $700 \mathrm{~nm}$. It should be noted that of the standard reference materials for measuring fluorescence yields, rhodamine 101 has the nearest match in emission range, however this is $600-650 \mathrm{~nm}$, longer than the chromophores' emission ranges. To compensate for this, the yields were determined as usual using the same excitation wavelength for both the reference and the standard. The yields were also calculated using the $550 \mathrm{~nm}$ and 500 $\mathrm{nm}$ excitation of the reference Rhodamine 101, so that the relative change in quantum yield could be determined for wavelengths where the rhodamine 101 reference solution does not absorb. 


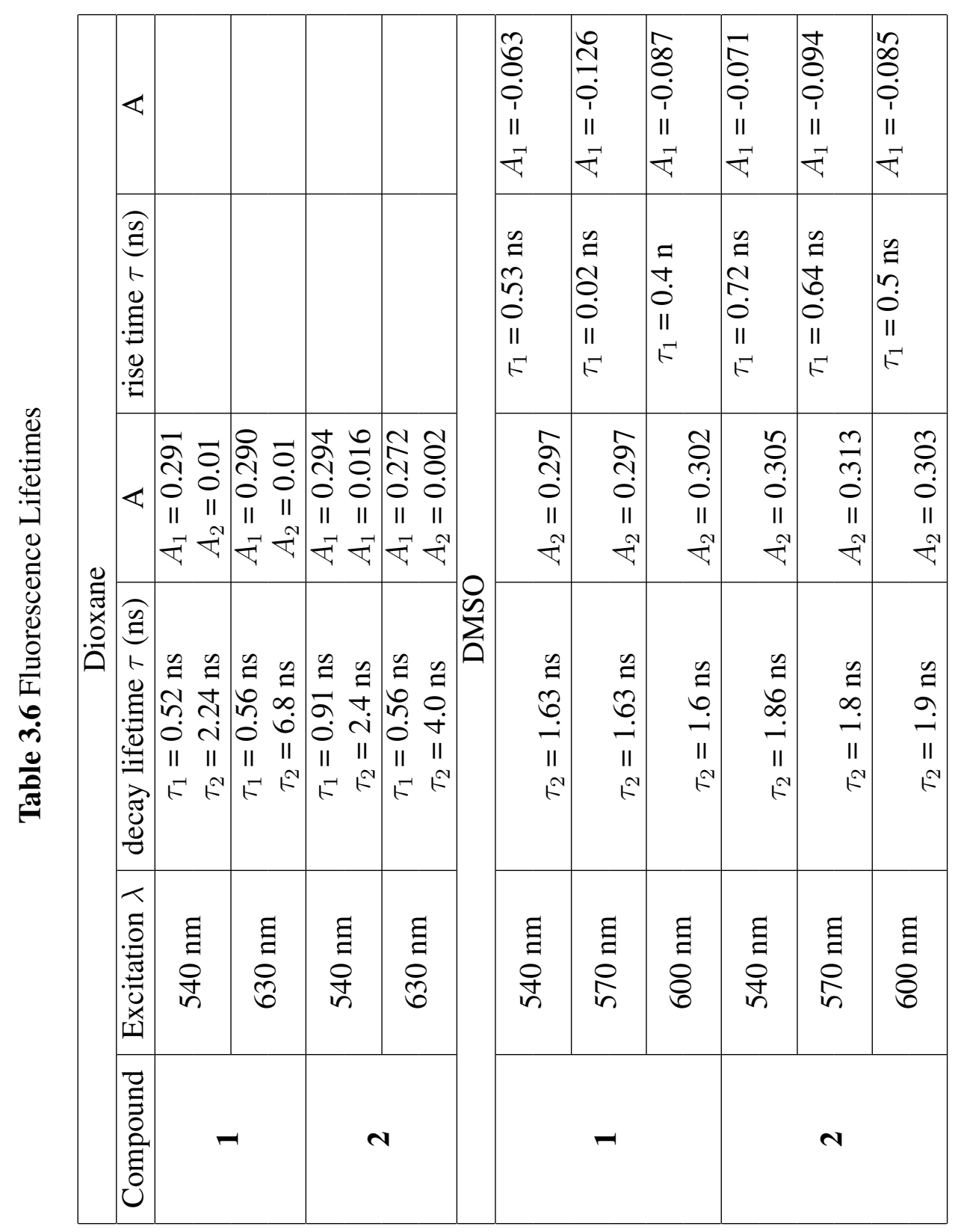


Table 3.7 Summary of Fluorescence Quantum Yields

\begin{tabular}{|c|l|lr|lr|}
\hline \multicolumn{5}{|c|}{ 1 In DMSO } \\
\hline Excitation Wavelength (nm) & $\begin{array}{l}\text { calculated with } \\
\text { same wavelength } \\
\text { peak }\end{array}$ & $\begin{array}{l}\text { calculated } \\
\text { calibrated } \\
\text { nm peak }\end{array}$ & $\begin{array}{l}\text { with } \\
\text { calculated }\end{array}$ & $\begin{array}{l}\text { with } \\
\text { calibrated } \\
\text { nm peak }\end{array}$ \\
\hline 500 & 0.12 & 0.20 & 0.11 \\
540 & 0.13 & 0.18 & 0.11 \\
550 & 0.12 & 0.18 & 0.11 \\
570 & 0.12 & 0.18 & 0.11 \\
590 & N/A & 0.19 & 0.11 \\
600 & N/A & 0.20 & 0.11 \\
\hline 500 & 0.41 & 0.47 & \\
\hline 540 & 0.59 & 0.57 & 0.41 \\
550 & 0.59 & 0.59 & 0.49 \\
570 & 0.69 & 0.66 & 0.57 \\
590 & - & 0.44 & 0.38 \\
600 & - & 0.47 & 0.41 \\
\hline
\end{tabular}




\section{Chapter 4}

\section{Detection of Rotamers by NMR}

\subsection{Introduction}

NMR spectroscopy is a useful technique for probing the chemical structure of molecule as the chemical shift of each proton is sensitive to its surrounding environment. Thus it is possible to detect the different chemical environments that the protons are in and deduce the structure of the molecule.

NMR studies of the parent chromophore $\mathbf{1 3}$ has shown that the compound can exist as two rotameric forms are present in DMSO as a mixture of s-cis and s-trans rotamers around the bond connecting the acceptor group to the $\pi$ bridge as shown in figure $4.1{ }^{71}$ It has been found that compound $\mathbf{1 4}$ with $\mathrm{R}_{3}=\mathrm{C}_{11} \mathrm{H}_{23}$ that in low polarity solvents such as toluene only one rotamer is present in solution, whereas in the more polar solvent DMSO two rotamers are apparent. ${ }^{46}$ The observation of only one rotamer in non polar solvents is of interest because aggregation is favoured in non-polar solvents and if the existence of rotamers is inhibited in low polarity solvents this would be expected to promote molecular aggregation due to favoured stacking of the molecules. 

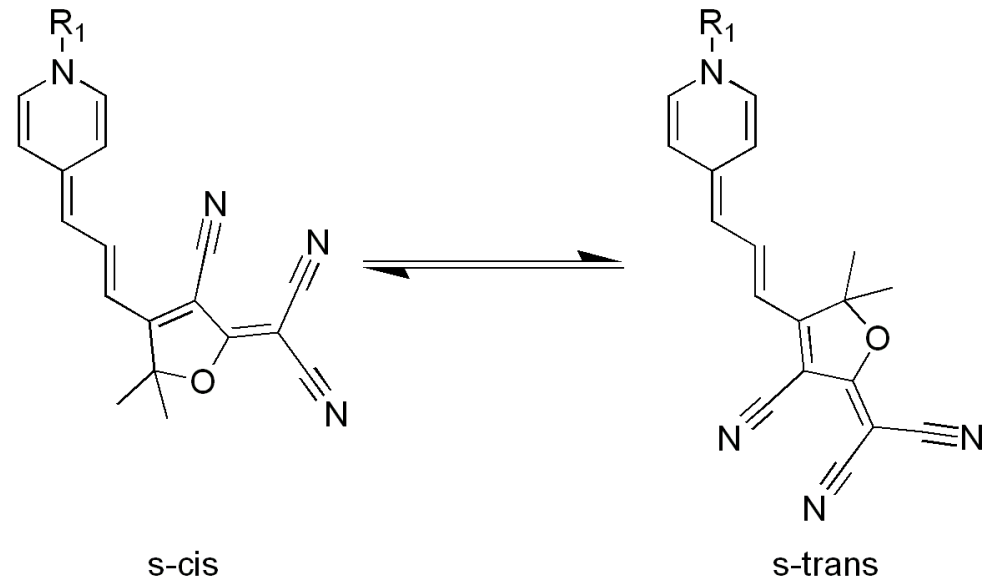

Figure 4.1 Two rotameric forms of a merocyanine chromophore showing s-cis and s-trans rotamers around the bond connecting the acceptor to the $\pi$ bridge.

\subsection{Results}

Compound 1 in d6-dmso displays two rotamers, indicated by a doublet at $5.75 \mathrm{ppm}[\mathrm{J}=$ $14.5 \mathrm{~Hz}]$, doublet at $6.35 \mathrm{ppm}[\mathrm{J}=14.5 \mathrm{~Hz}]$ and a doublet of doublets at $7.64 \mathrm{ppm}[\mathrm{J}=$ 10.4 Hz, 14.4 Hz]; the second rotamer has a doublet at 5.68ppm [ J = 12.4 Hz], a doublet at $6.28 \mathrm{ppm}[\mathrm{J}=16.4]$ and a doublet of doublets at $8.37 \mathrm{ppm}[\mathrm{J}=12.3 \mathrm{~Hz}, 12.4 \mathrm{~Hz}]$. These rotamer peaks are shown in figure 4.2. The integrals of these peaks show that the rotamers are present in roughly equal quantities with an average ratio of 1:0.9 between each peak. 




Figure 4.2 NMR spectra of compound 1 showing the presence of two rotamers.

Compound 15 has a $\mathrm{R}_{1}=\mathrm{C}_{10} \mathrm{H}_{21}$ with $\mathrm{R}_{2}=\mathrm{Bg}$ and $\mathrm{R}_{3}=\mathrm{C}_{11} \mathrm{H}_{23}$. In d6-DMSO $(\varepsilon=46.7)$ the first pair of doublets is present at $5.82 \mathrm{ppm}$ and $6.15 \mathrm{ppm}$. These two peaks have a ratio of 1:0.3, showing that one rotamer is dominant over the other in contrast to the 1:0.9 ratio in d6-DMSO of compound $\mathbf{1}$. In d6-acetone $(\varepsilon=21.4)$ chemical shift of these peaks is at $5.92 \mathrm{ppm}$ and $6.29 \mathrm{ppm}$ and their ratio is 1:0.05 and in $\mathrm{d} 8$-toluene only the peak at $5.84 \mathrm{ppm}$ is observed, suggesting that only one rotamer is present. 


\section{Chapter 5}

\section{Discussion}

The most unsubstituted compound $\mathbf{1 3}$, with $\mathrm{R}_{1}=\mathrm{R}_{3}=\mathrm{CH}_{3}$ and $\mathrm{R}_{2}=\mathrm{H}$ as shown in figure 5.1, is the core structure responsible for nonlinear optical activity. However the use of this compound alone is not practical for use in nonlinear devices as was discussed in the introduction, as it is insoluble in the solvents and conditions needed to process it into a polymer film. To address this issue a $\mathrm{C}_{10} \mathrm{H}_{21}$ group has been added to the $\mathrm{R}_{1}$ position in order to make the molecule more soluble (compound 1). However the problem of aggregation (see section 1.4.2) remains in this compound as is shown by the concentration dependent UV-Vis absorption study of compound 1. The bulky groups were added to this core structure as listed in table 2.1 in order to both improve the solubility of the compounds and reduce molecular aggregation. The spectroscopic studies were performed in order to establish the effect of the bulky groups on the aggregation of the compounds.

The results of these experiments are discussed below.

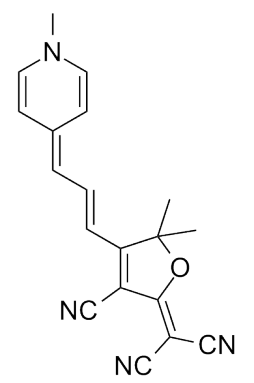

Figure 5.1 Basic merocyanine chromphore 13 


\subsection{Concentration dependent UV-Vis Studies}

Compounds 1 - 3 each have the $\mathrm{C}_{10} \mathrm{H}_{21}$ alkyl chain in the $\mathrm{R}_{1}$ position, while the $\mathrm{R}_{2}$ and $\mathrm{R}_{3}$ groups are varied. These three compounds show significant aggregation in dioxane with increasing chromophore concentration. This is evident from figures 3.2 to 3.4 which show a shorter wavelength shoulder band in each absorption spectrum whose extinction coefficient increases relative to the main band as concentration is increased. This was expected to be most prominent in compound 1 (figure 3.2) as it has the least bulky side groups $\left(\mathrm{R}_{1}=\mathrm{C}_{10} \mathrm{H}_{21}, \mathrm{R}_{2}=\mathrm{H}, \mathrm{R}_{3}=\mathrm{CH}_{3}\right)$. While the $\mathrm{C}_{10} \mathrm{H}_{21}$ group on the $\mathrm{R}_{1}$ position should provide some hindrance to stacking of the chromophore molecules into an aggregate, this group is located at one end of the molecule and so it is possible that it does not prevent the formation of aggregates as it is free to move away from the plane where dipole-dipole interactions between two chromophores would occur. Thus the bulky groups added to the $\mathrm{R}_{3}$ site in compounds $\mathbf{2}$ and $\mathbf{3}$ were designed to create an increased steric hindrance to aggregation by adding bulky groups around the acceptor end of the molecule. In compound $\mathbf{3}$, the addition of the bulky groups containing phenyl rings was also expected to have an effect on the aggregation behaviour of the compounds because of intermolecular $\pi-\pi$ interactions between the bulk groups in adjacent molecules.

The concentration dependence of the absorption spectra of $\mathbf{2}$ clearly shows a decrease in the relative size of the shoulder band compared to the main band (figure 3.3). Adding the two $\mathrm{C}_{11} \mathrm{H}_{23}$ groups to the $\mathrm{R}_{3}$ positions, combined with the $\mathrm{C}_{10} \mathrm{H}_{21}$ group on $\mathrm{R}_{1}$ has a significant effect on the extent of aggregation occurring in solution. With the addition of these $\mathrm{C}_{11} \mathrm{H}_{23}$ groups the chromophore now has bulky groups on both the electron 
donor and acceptor ends of the molecule, thereby hindering the approach of another chromophore molecule to form an aggregate.

The concentration dependent absorption spectra of compound $\mathbf{3}$ are shown in figure 3.4. Compound 3 has had the PhOBz group added to the $\mathrm{R}_{3}$ positions, while $\mathrm{R}_{1}=\mathrm{C}_{10} \mathrm{H}_{21}$ and $\mathrm{R}_{2}=\mathrm{H}$ remain unchanged. These PhOBz groups were added to the chromophore with the hope that the phenyl rings in this bulky group would dominate the interaction of the chromophore molecules, as was seen in the crystal structure of the acceptor with phenyl $\mathrm{R}_{3}=\mathrm{Ph}$ in figure $2.3^{46}$, in such a way that $\mathrm{H}$ aggregates would not be formed. However figure 3.4 shows a shoulder band at $560 \mathrm{~nm}$ that indicates that $\mathrm{H}$-aggregation is present and this peak is more dominant than in the spectrum of compound 2. The $\mathrm{C}_{11} \mathrm{H}_{21}$ chains appear to be much more effective at reducing aggregation than the $\mathrm{PhOBz}$ group. It may be that the PhOBz group is having an effect on the stacking of molecules, but in a way that still results in an $\mathrm{H}$-aggregate formation. It was hoped that the $\pi-\pi$ interactions of the phenyl rings that was seen in the phenyl acceptor would not be able to form into an ordered crystal, or would form a noncentrosymmetric crystal due to the extra bulk of the merocyanine backbone and the $\mathrm{R}_{1} \mathrm{C}_{10} \mathrm{H}_{21}$ making stacking of the phenyl rings difficult. As the aggregation peak is instead increased, it would appear that the phenyl rings on the $\mathrm{PhOBz}$ group are aiding in the stacking of the aggregate in an ordered Head to tail arrangement.

A second bulky group was added to the $R_{2}$ position in compounds 4,5 , and $\mathbf{6}$ to increase the steric hindrance through the central part of the molecule. In compounds 4 to 6 the $R_{1}$ position has a $\mathrm{CH}_{3}$ group as these compounds were sufficiently soluble in dioxane without 
the addition of the $\mathrm{C}_{10} \mathrm{H}_{21}$ chain. Compound $\mathbf{4}$ is otherwise equivalent to compound $\mathbf{1}$ with the addition of the bulky group at $\mathrm{R}_{2}$ and $\mathrm{R}_{3}=\mathrm{CH}_{3}$. Compound $\mathbf{5}$ is similarly equivalent to 2 , and compound $\mathbf{6}$ equivalent to 3 . A similar trend was seen in the aggregation behaviour of these molecules as was seen in compounds $\mathbf{1}-\mathbf{3}$. That is, compound $\mathbf{5}$ shows the least amount of aggregation of the three compounds in this section, showing only a small shoulder at $550 \mathrm{~nm}$ that does not change markedly with respect to the main absorption band as the concentration is increased. While compounds $\mathbf{4}$ and $\mathbf{6}$ have clearly defined absorption bands at $550 \mathrm{~nm}$ whose extinction coefficient increases as the extinction coefficient of the main band decreases.

A bulky group was also substituted at the $\mathrm{R}_{1}$ position. These compounds $\mathbf{7}$ to 9 are again equivalent to compounds $\mathbf{1}$ to $\mathbf{3}$ with $\mathrm{R}_{1}=\mathrm{Bg}, \mathrm{R}_{2}=\mathrm{H}$ and $\mathrm{R}_{3}=\mathrm{CH}_{3}$ in compound 7, $\mathrm{R}_{3}=\mathrm{C}_{11} \mathrm{H}_{23}$ in compound $\mathbf{8}$, and $\mathrm{R}_{3}=\mathrm{PbObz}$ in compound $\mathbf{9}$. A similar trend was again shown. It should be noted however that these compounds have a low solubility. Enough could be dissolved for the spectroscopic studies, but the limited solubility makes them less suitable for casting into polymer films. The limited solubility is also the likely cause of the observed extinction coefficients of these compounds being much lower than those of compounds $\mathbf{1}$ to $\mathbf{6}$. This may be because although solutions were only measured when there was no visible undissolved chromophore, the actual concentration of the compound in solution may have been slightly lower than the calculated concentration due to only partially dissolved chromophore.

It can be seen from a figures 3.2 to 3.10 that the subsitutent with the largest effect on the formation of aggregates is with the $\mathrm{C}_{11} \mathrm{H}_{23}$ group added to the acceptor moiety of the 
merocyanine. The bulky group added to the $\mathrm{R}_{2}$ position (middle of the molecule) also has a noticeable effect of inhibiting aggregation. From these observations it is expected that the compound with $\mathrm{R}_{2}=\mathrm{Bg}$ and $\mathrm{R}_{3}=\mathrm{C}_{11} \mathrm{H}_{23}$ will show the least aggregation. This appears to be confirmed by figure 3.6 (compound 5), which shows only a small aggregation band.

\subsection{Fluorescence}

The normalised fluorescence spectra of compound $\mathbf{1}$ are shown in a range of solvents (DMSO, acetonitrile, THF, and chloroform) in figures 3.19 to 3.22. These figures show the emission spectrum of the chromophore (black) resulting from excitation at a wavelength near the maximum of the main absorption band, and the excitation spectrum of the chromophore (red) recorded at the emission maximum. The normalised absorption spectrum of the sample is also shown in blue and the excitation and emission wavelengths used for each sample are given in the figure. These spectra show that the fluorescence behaviour is similar for all the solvents used. Each fluorescence spectra shows a mirrorimage symmetry of the emission spectra with the absorption spectra, and the excitation spectrum that is of similar shape to that of the absorption spectrum. This behaviour is similar in the other chromophores 2 to 9 . A summary of the emission maxima is given in table 3.5 .

The exception to this mirror-image pattern is in the fluorescence spectra of the merocyanines in 1,4-dioxane. The fluorescence spectra of compounds $\mathbf{1}$ to $\mathbf{9}$ are shown in figures 
3.23 to 3.28. In 1,4-dioxane the absorption spectrum of the chromophore show a shoulder at shorter wavelengths to the main absorption band. As has been discussed above this absorption shoulder is attributed to the absorption of an $\mathrm{H}$-aggregate of the chromophore molecules. Neither the fluorescence emission (black) or excitation spectra (red) in figures 3.23 to 3.28 show a similar shoulder. The excitation spectra have maxima at the same wavelength as the main peak of the absorption band, indicating that monomeric form of the chromophore is solely responsible to the fluorescence emission; the contribution to fluorescence from molecular aggregates is negligible and excitation into the aggregate band does not result in a separate fluorescence band. For example, excitation of compound 1 at $545 \mathrm{~nm}$ results in a weak emission at $662 \mathrm{~nm}$, identical to the emission spectrum of the solution when excited at the main band $(632 \mathrm{~nm})$.

Fluorescence lifetime measurements of compounds $\mathbf{1}$ and $\mathbf{2}$ were performed in dioxane and DMSO. Dioxane was used because of the aggregation seen in the concentration studies of the chromophores in this solvent. The fluorescence lifetimes of the chromophores were also measured in DMSO to provide a comparison of the fluorescence of the chromophore in a solvent where aggregation was not observed. These results were summarised in table 3.6.

In DMSO both compounds show a single exponential fluorescence decay with lifetimes of $1.16 \mathrm{~ns}$ for compound $\mathbf{1}$ and $1.18 \mathrm{~ns}$ for compound $\mathbf{2}$. The fluorescence lifetimes were measured when exciting at $570 \mathrm{~nm}$, a wavelength within the main absorption band, and also at excitation wavelengths of $540 \mathrm{~nm}$ and $600 \mathrm{~nm}$. These second two wavelengths were used to excite the sample on either side of the absorption band. It was found that 
for both compounds $\mathbf{1}$ and $\mathbf{2}$, the fluorescence decay kinetics were independent of the excitation wavelength. 1 has a decay of $\tau_{2}=1.63 \mathrm{~ns}$ with both 540 and $570 \mathrm{~nm}$ excitation, and $\tau_{2}=1.60 \mathrm{~ns}$ at $600 \mathrm{~nm}$. Compound 2 has a decay of $\tau_{2}=1.86 \mathrm{~ns}$ with $540 \mathrm{~nm}$ excitation, $\tau_{2}=1.8 \mathrm{~ns}$ with $570 \mathrm{~nm}$ excitation, and $\tau_{2}=1.9 \mathrm{~ns}$ with $600 \mathrm{~nm}$ excitation, again the decay kinetics are essentially independent of excitation wavelength.

When the fluorescence lifetime measurements of the two compounds were performed in 1,4-dioxane a sum of two exponential decays were observed. The fluorescence decays were in this case measured at two excitation wavelengths; at $630 \mathrm{~nm}$ to excite into the main absorption band, and at $540 \mathrm{~nm}$ to excite into the minor side absorption band. The two exponential decay suggests that the second decay component is due to the molecular aggregate formed in the dioxane solution. The decay is dominated by the short $(<1 \mathrm{~ns})$ component independent of the excitation wavelength.

The two decays in dioxane show compared to only one decay exponential in DMSO suggests that the extra decay in dioxane is due to the presence of the aggregate. In DMSO the decay behaviour is consistent, with similar lifetimes and pre-exponential factors across each wavelength measured, supporting the existence of fluorescence only from one energy level. In dioxane the major decay in at each excitation wavelength for both compound 1 and compound 2 is the shorter component, $\tau_{1}$. The pre-exponential parameter $A_{1}$ stays constant at approximately 0.3 at both excitation wavelengths, showing that this fluorescence decay is independent of the excitation wavelength. It is thus proposed to be the fluorescence from the monomeric form of the chromophore. The presence of this decay when the sample is excited within the aggregate's absorption band at $540 \mathrm{~nm}$ 
because at this wavelength there is still absorption into the overlapping monomer band occurring. The second, minor component of the exponential decay $\tau_{2}$ is a longer decay and is also present at both excitation wavelengths. In this case the pre-exponential factor is larger by a factor of 10 when the excitation wavelength is $540 \mathrm{~nm}$ (aggregate band) over the value in the monomer band $(630 \mathrm{~nm})$ this suggests that this fluorescence decay is due to the aggregate.

\subsection{Rotamers}

It has been previously observed by NMR studies that compound $\mathbf{1}$ in solution is present as a mixture of rotational isomers. ${ }^{46,71}$ In d-DMSO compound $\mathbf{1}$ displays doublets at 5.75 ppm and $5.68 \mathrm{ppm}$; doublets at 6.35 and $6.28 \mathrm{ppm}$, and two doublet of doublets at 7.64 and $8.37 \mathrm{ppm}$. Each pair is present in a ratio of 1:0.9 suggesting that both of the rotamers are present in roughly equal amounts. This is likely to hinder aggregation as molecules that are in different rotamers will not stack as easily into an aggregate as if they are all in the same configuration.

Compound $\mathbf{5}$ shows the least aggregation in the concentration studies discussed above and has $\mathrm{C}_{11} \mathrm{H}_{23}$ alkyl chains on the $\mathrm{R}_{3}$ position that aid solubility and so an attempt was made to measure the NMR of $\mathbf{5}$ in a range of solvents. However this compound was still not soluble enough to obtain a clear NMR measurement. Compound $\mathbf{1 5}$ has a $\mathrm{C}_{10} \mathrm{H}_{21}$ group added to the $\mathrm{R}_{1}$ position to further increase the solubility. With $\mathrm{R}_{2}=\mathrm{Bg}$ and $\mathrm{R}_{3}=\mathrm{C}_{11} \mathrm{H}_{23}$ this compound is the same as compound $\mathbf{5}$, apart from the change to the $\mathrm{R}_{1}$ position, 
and is sufficiently soluble to compare the ratios of the rotamers present in d6-DMSO, dacetone, and d8-toluene. In DMSO the first double pair is present at $5.82 \mathrm{ppm}$ and 6.15 ppm. These two peaks have a ratio of 1:0.3, showing that one rotamer is dominant over the other in contrast to the 1:0.9 ratio in d6-DMSO of compound 1 . In acetone the ratio of the peaks at $5.92 \mathrm{ppm}$ and $6.29 \mathrm{ppm}$ is 1:0.05 and in d-toluene only the peak at 5.84 ppm is observed, suggesting that only one rotamer is present.

In d6-DMSO the polar solvent environment can stabilize the zwitterionic form of the molecule. This means that the bond around which the rotation occurs (see figure 2.37) has more double bond character than if the molecule is in the neutral state where it is a single bond. Thus there would be a large energy barrier to rotation about this bond and so the molecules may be locked into either the cis or trans rotamer and not transfer between the two. This would result in seeing the two distinct rotamer peaks in the d6DMSO NMR. The bulky groups on compound $\mathbf{5}$ in the $\mathrm{R}_{1}$ and $\mathrm{R}_{2}$ positions would have a hindering effect on this rotation, so if one rotamer is more likely to form in the synthesis of the molecules, it would be unable to rotate to the other isomer, explaining the unequal ratios of rotamers in this compound compared to compound $\mathbf{1 .}$

In lower polarity solvents if the neutral form of the molecule is the more stabilized then rotation can occur more freely around the single bond, so if one rotamer is energetically favoured, it is likely to be able to isomerise to that rotamer so only one peak is observed in the NMR. 


\subsection{Solvatochromism}

The aggregation studies discussed in section 3.1 were performed in a number of solvents. The polarity of the solvents used in the concentration dependence study is important because the solvating environment has an effect on the tendency of chromophores aggregate formation, or instead, favour their monomeric form in solution. In a low polarity solvent a chromophore with a zwitterionic ground state is not stabilised as much as it would be in a more polar solvent, and so 1 ,4-dioxane $(\varepsilon=2.304)$ is the solvent where the largest amount of aggregation is expected to be seen whereas dimethyl sulfoxide ( $\varepsilon=46.7$ ) is the most polar solvent used and aggregation is not expected to be observed. The concentration studies performed in DMSO and Chloroform confirmed that aggregation was not seen in these solvents, with typical examples of the observed results shown in figures 3.11 and 3.12 showing no aggregation peak or concentration dependence.

As well as the absence of a shoulder band in DMSO compared to dioxane, two other differences are apparent in different solvents. There is a blue shift in the position of the spectral maximum, $\lambda_{\max }$, as the polarity of the solvent is increased, and the band shape changes from a narrow band in dioxane and chloroform to a broad band in DMSO. This broadening of the absorption band can be clearly seen in figure 3.16 which shows the absorption spectrum of compound $\mathbf{6}$ in a series of chloroform-acetonitrile solvent mixtures. As the molar fraction of acetonitrile is increased in the solution the absorption maximum of $\mathbf{6}$ shifts towards the blue end of the visible spectrum (also shown in figure 3.17) and the absorption band broadens. 
The blue shift of $\lambda_{\max }$ that occurs with compounds $\mathbf{1}$ - 9 is typical for dyes that have a ground state with highly delocalizable electrons. In highly polar solvents the solvating environment can stabilize the zwitterionic resonance form of the dye, resulting in a larger $\Delta E=E_{E}-E_{G}$ between the ground and excited states than in a low polarity solvent. At least part of the broadening of the absorption band can be explained in terms of the resonance forms the merocyanine ground state that can exist, ranging from the neutral molecule to the fully zwitterionic form. In medium polarity solvents such as chloroform the absorption spectrum shows a narrow cyanine-type absorption band suggesting that the ground state has contributions from both the neutral and zwitterionic state. As solvent polarity increases, the increase in $\Delta E$ and the broadening of the absorption band indicate a shift in the ground state to a more zwitterionic state.

The change in absorption maxima of $\mathbf{1}$ - $\mathbf{9}$ and the accompanying change in the emission maxima of these compounds is shown in figures 3.14 and 3.15. Figure 3.14 shows the solvatochromism using the solvent's dielectric constant, $\epsilon_{r}$, as a measure of solvent polarity. When the absorption maximum is plotted against $E_{T}(30)$, an empirical scaled based of the solvatochromism of a betaine dye, the change in $\lambda_{\max }$ is approximately linear. Two notable exceptions to this, circled in blue, are the absorption maxima of the chromophores in chlorinated solvents and circled in red is the absorption maximum in methanol. These absorption maxima occur at lower energy than the trend shown in the other solvents. It is likely that this is occurring due to a specific solvent-solute interactions in these solvents, such as hydrogen bonding between the methanol and the chromophore. To compare the behaviour of the absorption maxima while eliminating specific solvent-solute interactions, absorption spectra were measured in binary solvent 
mixtures. Figure 3.17 shows $\lambda_{\max }$ for a range of polarities determined by changing the molar fraction of the two solvents. This figure shows that the solvatochromic behaviour of $\mathbf{6}$ in both acetonitrile-chloroform mixtures and acetonitrile-dioxane mixtures is similar although chloroform is a chlorinated solvent and dioxane is an ether. The overall polarity of the solvating environment seems to have a more dominant effect on the $\lambda_{\max }$ than any specific solvent-solute interactions.

As well as changes in the absorption maximum, there are also changes in the relative distribution of vibrionic transition probabilities (intensities) observed in the absorption spectra of the molecules in different polarity solvents. This phenomenon has been observed in some molecules possessing degenerate, or nearly degenerate, excited electronic states. ${ }^{65}$ It has also been observed in molecular dimers where the identical excited states of the partners of molecular pairs interact. As a result of intramolecular vibrational coupling of excited states within a molecule or by intermolecular vibrations of the partners of a molecular pair moving with respect to one another, the energies of the states are perturbed and the degeneracy removed. This behaviour has been referred to as pseudoJahn-Teller splitting. ${ }^{66-68}$ In the merocyanines studied in this project, in solvents where an alternation in the vibronic structure was observed, there was no evidence of molecular dimerisation/aggregation because no change in the shape of the vibronic distribution was observed as the concentration of solute was increased from a very low concentration of $\sim 1 \times 10^{-4}$ mol.dm ${ }^{-3}$ to a high concentration of $\sim 1 \times 10^{-6}$ mol.dm ${ }^{-3}$ approaching saturation. In these cases the change in vibronic structure can be accounted for in terms of intra-molecular vibrational coupling in the following way - the "pseudo-Jahn Teller effect". ${ }^{67,68}$ In a polar solvent, the first two excited states of these merocyanines are 
postulated to be nearly degenerate. An out-of-plane vibration can couple/mix these $\pi \pi^{*}$ and $\mathrm{n} \pi^{*}$ states and, from first-order perturbation theory, there is a concomitant lifting of the degeneracy, i.e. the states split in energy. This behaviour has been invoked previously for some other molecules, containing either $\mathrm{N}$-hetrocycles or carbonyl groups which possess two close-lying $\pi \pi^{*}$ and $\mathrm{n} \pi^{*}$ excited singlet states, to explain anomalous spectroscopic observations in various solvents ${ }^{66,69}$ These two types of electronic states interact with polar and/or hydrogen bonding solvents in different ways. The $\pi \pi^{*}$ states are more polarizable and are stabilised by the higher dielectric molecular environment associated with a polar solvent whereas the lone pair $n$ electrons are subject to specific interactions with hydrogen bonding solvents. ${ }^{66}$. As a result, depending on the molecular environment, the energies of these two states move relative to each other and to the ground state. Therefore, in certain solvents, when the energies of the two excited states are sufficiently close, they exhibit a splitting of the first two excited states as a result of mixing of these states via out-of-plane vibrational coupling.

The lowest energy perturbed excited state can be shown to have a lower vibrational frequency and the upper state a higher frequency than the zero order states. This is shown schematically in figure 5.2. One consequence of this is for absorption into a vibrational level with a higher vibrational quantum number than is the case for the zero order, unperturbed excited singlet as indicated in the figure. This results in a redistribution of vibronic transition probabilities in the lowest energy absorption band and the most probable vibronic transition, i.e. that with the greatest FC overlap factor, shifting to higher energy as the solvating power of the solvent increases. 


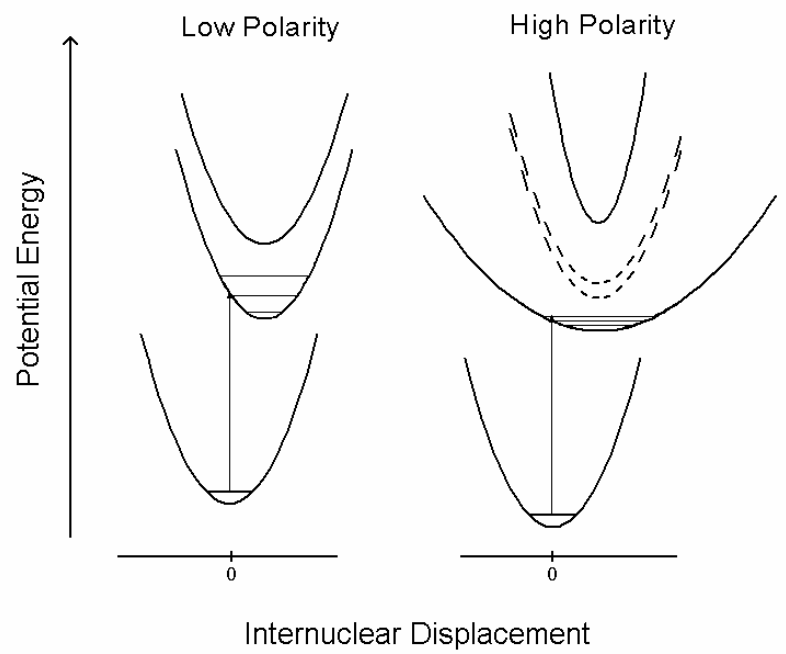

Figure 5.2 Pseudo Jahn-Teller effect on the energies of vibronic states. The diagram on the left shows the unperturbed energy levels in a non-polar, aprotic solvent. The high polarity/solvating case is shown on the right where the nearly degenerate excited energy states (dashed lines) have coupled via vibrational modes lifting the degeneracy (solid lines). 


\section{Chapter 6}

\section{Experimental}

All solutions were made using spectrophotometric or HPLC grade solvents purchased from Sigma-Aldrich and the merocyanine non-linear compounds synthesised by the Photonics group at Industrial Research Ltd. All measurements were performed using matched quartz absorption or fluorescence cells purchased from Starna.

\subsection{UV-Vis Absorption Spectroscopy}

The UV-Vis absorption spectra of compounds $\mathbf{1}-\mathbf{9}$ were measured over a range of concentrations from $1 \times 10^{-6}$ mol.dm ${ }^{-3}$ to $2 \times 10^{-5}$ mol.dm ${ }^{-3}$. This concentration range gives a variation in absorbance at the absorption maxima from 0.05 to 1.00 in a $1 \mathrm{~cm}$ cuvette. UV-Vis absorption measurements were performed on a Lambda-1050 dual-beam spectrometer from Perkin Elmer. All measurements were performed using matched quartz absorption cells purchased from Starna.

Stock solutions of the chromophores in each solvent were made up to $2.5 \mathrm{~mol} . \mathrm{dm}^{-3}$. Solutions with a range of concentrations were made up by sequentially adding 50 $\mu \mathrm{L}$ aliquots of the stock solution to $1 \mathrm{~mL}$ of solvent. This gives solutions with a volume ranging from $1050 \mu \mathrm{L}-1700 \mu \mathrm{L}$. The UV-Vis absorption spectra were recorded immediately after each successive addition of stock solution. 
Solvents were chosen to cover a range of polarities (dielectric constants, $\epsilon_{r}$ ), although this choice was limited by the solubility of the chromophores. In order to cover a range of solvent polarities the experiment was performed in 1,4-dioxane, $\left(\epsilon_{r}=2.2\right)$; chloroform, $\left(\epsilon_{r}=4.81\right)$; tetrahydrofuran, $\left(\epsilon_{r}=7.47\right)$; and dimethyl sulfoxide, $\left(\epsilon_{r}=46.7\right)$.

Binary solvent mixtures were prepared by mixing the two solvents in the desired molar ratio. The polarities of the binary solvent mixtures were taken from the Static Dielectric Constants of Pure Liquids and Binary Liquid Mixtures by Wohlfarth. ${ }^{60}$ The relevant solvent polarity tables have been reproduced in table 3.4.

\subsection{Steady-State Fluorescence Spectroscopy}

Steady-state emission and excitation fluorescence spectra were performed on a Horiba Fluorolog Modular Spectrofluorometer or a Varian Cary Eclipse spectrofluorometer. A fluorescence spectrometer records steady-state fluorescence spectra in two modes; either in the form of an emission spectrum or an excitation spectrum. These are steady-state measurements performed with constant illumination (excitation) of the sample. The emitted light is detected at an angle to the exciting light (usually $90^{\circ}$ ). An emission spectrum is recorded using a specific excitation wavelength, and the detector records the spectral distribution of the emitted light (fluorescence). To record an excitation spectrum the detector is held at a specific emission wavelength while the excitation wavelength is varied to give a spectrum showing the dependence of the emission intensity on the excitation wavelength. 


\subsection{Fluorescence Lifetime Measurements}

Fluorescence lifetime decays were measured using Time-Correlated Single Photon Counting (TCPSC), performed at the School of Chemistry at the University of Melbourne. The excitation source used was a Fianium, SC450-PP-HE, super-continuum laser operating at a pulse repetition rate of $2 \mathrm{MHz}$ with pulses of approximately 100 ps duration with $\mathrm{nJ} / \mathrm{nm}$ pulse energy.The desired excitation wavelengths $(540,570,600,630 \mathrm{~nm})$ were selected from the super-continuum output using a dispersive prism and mechanical slit arrangement based on the design of Hell et al. ${ }^{70}$ The synchronisation signal was derived using a fast Becker and Hickl photodiode detecting a small part of the residual supercontinuum. An Eldy EM-132 microchannel plate photomultiplier tube was used to detect the emission following a polarisation analyser set at the magic angle, F1 collection lens and a obin Yvon $\mathrm{H}_{20}$ emission monochromator.Non-linear least square iterative reconvolution used the instrument response function acquired by scattering the excitation light with a dilute solution of colloidal silica.

\subsection{Quantum Yield Measurements}

Quantum yields were measured using the Horiba Fluorolog Modular Spectrofluorometer. The comparative method of quantum yield determination was used with Rhodamine 101 and Rhodamine $\mathrm{G}$ as standards. Rhodamine 101 was chosen as it has the longest wavelength absorption and fluorescence emission of the IUPAC accepted standards available, and Rhodamine $G$ was used as it has a similar absorption spectrum. By 
comparing the corrected emission spectra of the unknown compound to that of a known standard the quantum yield $(\Phi)$ can be determined using equation 6.1 , where $\Phi_{u}$ is the quantum yield of the unknown, $\Phi_{s}$ is the quantum yield of the standard, $A_{s}$ and $A_{u}$ are the absorption of the standard and unknown respectively at the excitation wavelength, $F_{s}$ and $F_{u}$ are the area under the corrected fluorescence spectrum of the standard and unknown, and $n_{s}$ and $n_{u}$ are the refractive index of the solvents used for the standard and unknown.

$$
\Phi_{u}=\Phi_{s} \frac{A_{s}}{A_{u}} \frac{F_{u} n_{u}^{2}}{F_{s} n_{s}^{2}}
$$




\section{Chapter 7}

\section{Conclusions and Future Work}

\subsection{Conclusions}

- Compounds 1 - 9 have been found to form aggregates in solution in the low polarity solvents used in this work (1,4-dioxane and toluene). While aggregation was seen in all merocyanine compounds in these solvents, the substituent groups added to the $R_{1}, R_{2}$ and $R_{3}$ positions were found to affect the degree of aggregation as follows.

- The $\mathrm{C}_{11} \mathrm{H}_{23}$ alkyl chain subsitiuent groups on the $\mathrm{R}_{3}$ position has the largest effect on aggregation, with compounds $\mathbf{2 , 5}$, and $\mathbf{8}$ showing less aggregation than compounds with a $\mathrm{CH}_{3}$ group on the $\mathrm{R}_{3}$ position.

- A bulky group substituted on either the $R_{1}$ or $R_{2}$ position may reduce aggregation slightly but does not have as pronounced an effect as the $\mathrm{C}_{11} \mathrm{H}_{23}$ group on the $\mathrm{R}_{3}$ position.

- The PhOBz group in the $\mathrm{R}_{3}$ position, however, favours the formation of aggregates.

- Compounds 1 - 9 are "RHS", NLO chromophores and show negative solvatochromism as is usual for these molecules, indicating that the compounds have a zwitterionic ground state.

- Fluorescence of compounds $\mathbf{1}$ - 9 in 1,4-dioxane shows a non-mirror-image 
spectrum suggesting that the aggregate reduces fluorescence.

- The fluorescence lifetime decays of compounds $\mathbf{1}$ and $\mathbf{2}$ show a single exponential decay in DMSO where aggregation is not observed. In 1,4-dioxane a 2 exponential fluorescence decay is observed which is attributed to fluorescence from the aggregate as well as from the monomer.

\subsection{Future Work}

- Aggregation has been found to be reduced in solution by the introduction of bulky substituent groups. The next step is to measure the $|\mu . \beta|$ values of these chromophores in thin polymer films, the environment in which they would be used. If the alkyl chains on compound $\mathbf{5}$ also reduce the extent of aggregation in a film then higher $\beta$ values should be measured than for compound $\mathbf{1}$.

- Further fluorescence lifetime studies to investigate the rise time and two decay components of the fluorescence decay in dioxane. Comparing these results to other solvents where aggregation is seen such as toluene to confirm that the second decay component is due to the molecular aggregate.

- Determination of the level of aggregation occurring in a host-guest film is difficult due to broadening of the absorption spectra. However measurement decay kinetics of the fluorescence spectra of thin films could be used to detect and study the presence of aggregates.

- NMR studies of compounds 1 have suggested the presence of s-cis and s-trans 
rotamers in d6-DMSO ${ }^{46,71}$ while in lower polarity solvents only one rotamer is observed. Further NMR studies of compounds $\mathbf{1}$ - 9 comparing the rotamer behaviour between different polarity solvents and high temperature NMR would give information on which rotamer is the thermally favoured isomer. This information could help in suggesting processing methods which encourage the presence of both rotamers, hence making stacking of into the aggregate more difficult than in solutions where only one rotamer is present (such as in dioxane). 


\section{References}

1. Teshome, A.; Kay, A. J.; Woolhouse, A. D.; Clays, K.; Asselberghs, I.; Smith, G. J. Opt. Mat. 2009, 31, 575-582.

2. Dalton, L. R. Polymers for Photonic Applications I, Adv. in Polymer Sci.; SpringerVerlag: Berlin, 2002; Chapter Nonlinear Optical Polymeric Materials: From Chromophore Design to Comercial Applications., pp 1-86.

3. Wallace, J. Laser Focus World 2011, 47, 40-50.

4. Dalton, L. R.; Harper, A. W.; Ghosn, R.; Steier, W. H.; Ziari, M.; Fetterman, H.; Shi, Y.; Mustacich, R. V.; Jen, A. K.-Y.; Shea, K. J. Chem. Mater. 1995, 7, 10601081.

5. Wurthner, F.; Wortmann, R.; Meerholz, K. Chemphyschem 2002, 3, 17-31.

6. Haurylau, M.; Chen, G.; Chen, H.; Zhang, J.; Nelson, N.; Albonesi, D.; Friedman, E.; Rauchet, P. IEEE J. J. Select. Topics Quantum Electron. 2006, 12, 1699-1705.

7. Ma, J.; Povinelli, M. L. Curr. Opin. Solid State Mater. Sci. 2012, 16, 82-90.

8. Kurt, H. App. Opt. 2011, 50, 5256-5262.

9. Dalton, L. R.; Steier, W. H.; Robinson, B. H.; Zhang, C.; Ren, A.; Garner, S.; Chen, A.; Londergan, T.; Irwin, L.; Carlson, B.; Fifield, L.; Phelan, G.; Kincaid, C.; Amend, J.; Jen, A. J. Mater. Chem. 1999, 9, 1905-1920.

10. Ma, H.; Jen, A. K.-Y.; Dalton, L. R. Adv. Mater. 2002, 14, 1339-1365.

11. Dalton, L. R. Opt. Eng. 2000, 39, 589-595.

12. Quan, F. J. Opt. 2012, 024001, 1-4.

13. Williams, D. J. Angew. Chem. Int. Ed. 1984, 23, 690-703.

14. Prasad, P. N.; Reinhardt, B. A. Chem. Mater. 1990, 2, 660-669.

15. Wampler, R. D.; Moad, A. J.; Moad, C. W.; Heiland, R.; Simpson, G. J. Acc. Chem. Res. 2007, 40, 953-960.

16. Smith, F. G.; King, T. A. Optics and Photonics: An Introduction; Wiley, 2004.

17. Sun, S.-S., Dalton, L. R., Eds. Introduction to Organic Electronic and Optoelectronic Materials and Devices; CRC Press, 2008.

18. Christodoulides, D. N.; Khoo, I. C.; Salamo, G. J.; Stegeman, G. I.; Van Stryland, E. W. Adv. Opt. and Photonics 2010, 2, 60-200.

19. Marder, S. R.; Gorman, C. B.; Meyers, F.; Perry, J. W.; Bourhill, G.; Bredas, J.-L.; Pierce, B. M. Science 1994, 265, 632-635.

20. Janner, D.; Tulli, D.; Garcia-Granda, M.; M., B.; Pruneri, V. Laser \& Photon Rev. 2009, 3, 301-313. 
21. Shi, Y.; Zhang, H.; Bechtel, J. H.; Dalton, L. R.; Robinson, B. H.; Steier, W. H. Science 2000, 288, 119-122.

22. Nie, W. Adv. Mater. 1993, 5, 520-544.

23. Bhat, H. L. Bull. Mater. Sci. 1994, 17, 1233-1249.

24. Fujara, F.; Stockmann, H.-J.; Ackermann, H.; Buttler, W.; Dorr, K.; Grupp, H.; Heitjans, P.; Kiese, G.; Korblein, A. Z. Physik. B. 1980, 37, 151-161.

25. Marder, S. R.; Perry, J. W.; Bourhill, G.; Gorman, C. B.; Tiemann, B. G.; Mansour, K. Science 1993, 261, 186-189.

26. Marder, S. R.; Beratan, D. N.; Cheng, L. T. Science 1991, 252, 103-104.

27. Abdel-Halim, S. T. J. Chem. Soc. Faraday Trans. 1993, 89, 55-57.

28. Shindy, H. A. Coloration Tech. 2007, 123, 298-305.

29. Sainudeen, Z.; Ray, P. C. J. Phys. Chem. A 2005, 109, 9095-9103.

30. Ray, P. C.; Bonifassi, P.; Leszczynski, J. J. Phys. Chem. A. 2006, 110, 8963-8969.

31. Gayvoronsky, V. Y.; Uklein, A.; Vishnyakov, E.; Brodyn, M. S.; Grynko, D.; Kachkovsky, O. Mol. Cryst. Liq. Cryst. 2011, 535, 132-139.

32. Rao, V. P.; Jen, A. K. Y.; Wong, K.-Y.; Drost, K.; Mininni, R. M. Proc. SPIE 1992, 1775.

33. Dirk, C. W.; Katz, H. E.; Schilling, M. L. Chem. Matter. 1990, 2, 700-705.

34. Jen, A. K.; Wong, K. Y.; Rao, V.; Pushkara, V.; Drost, K.; Cai, Y. M.; Caldwell, B.; Mininni, R. M. Mater. Res. Soc. Symp. Proc. 1994, 328, 413.

35. Marder, S. R.; Cheng, L. T.; Tiemann, B. G.; Friedli, A. C.; Blanchard-Desce, M.; Perry, J. W.; Skindhoj, J. Science 1994, 263, 511-514.

36. Jenekhe, S. A., Wynne, K. J., Eds. Photonic and Optoelectronic Polymers; The American Chemical Society, 1997.

37. Rau, I.; Armatys, P.; Chollet, P. A.; Kajzar, F.; Y., B.; Andraud, C. Phys. Lett. 2007, 442, 329-333.

38. Marks, T. J.; A., R. M. Angew. Chem. Int. Ed. 1995, 34, 155-173.

39. Eisfeld, A.; Briggs, J. S. Chem. Phys. 2006, 324, 376-384.

40. Kasha, M. Radiation Research 1963, 20, 55-71.

41. Kato, N.; Saito, K.; Uesu, Y. Ferroelectrics 2000, 238, 245-252.

42. Wurthner, F.; Yao, S.; Debaerdemaeker, T.; Wortmann, R. J. Am. Chem. Soc. 2002, 124, 9431-9447.

43. Smith, G. J.; Middleton, A. P.; Clarke, D. J.; Teshome, A.; Kay, A. J.; Bhuiyan, M. D. H.; Asselberghs, I.; Clays, K. Opt. Mat. 2010, 32, 1237-1243. 
44. Clarke, D. J.; Middleton, A.; Teshome, A.; Bhuiyan, M. D. H.; Ashraf, M.; Gainsford, G. J.; Asselberghs, I.; Clays, K.; Smith, G. J.; Kay, A. J. Proc AIP Conference Proceedings, AMN-4, Dunedin, New Zealand 2009, AMN4, 90-93.

45. Fernandez, G.; Stolte, M.; Stepanenko, V.; Wurthner, F. Chem. Eur. J. 2012, 19, 206217.

46. Anderson, J. Zwitterionic Organic Non Linear Optical Chromophores With Modified Acceptor Groups. M.Sc. thesis, School of Chemical and Physical Sciences, Victoria University of Wellington, 2009.

47. Jelley, E. E. Nature 1936, 137, 1009-1010.

48. Scheibe, G. Angew. Chem. 1936, 49, 563.

49. Scheibe, G. Angew. Chem. 1937, 50, 212-219.

50. Frank, J.; Teller, E. J. Chem. Phys. 1938, 6, 861-872.

51. Frenkel, J. Phys. Rev. 1931, 37, 17-44.

52. Frenkel, J. Phys. Rev. 1931, 37, 1276-1294.

53. Kasha, M.; Rawls, H. R.; El-Bayoumi, M. A. Int. U. Pure Appl. Chem. 1965, 11, 371-392.

54. Palsson, L.-O.; Szablewski, M.; Roberts, A.; Masutani, A.; Love, G. D.; Cross, H., Graham; Bloor, D.; Kay, A. J.; Woolhouse, A. D.; Masutani, A.; Yazuda, A. Mol. Cryst. Liq. Cryst. 2003, 402, 43/[279]-53/[289.

55. Reichardt, C. Solvents and Solvent Effects in Organic Chemistry; Wiley-VCH, 2003.

56. Reichardt, C. Chem. Rev. 1994, 94, 2319-2358.

57. Patnaik, L. N.; Sahu, B. J. Soln. Chem. 1994, 23, 1317-1330.

58. Wurthner, F.; Archetti, G.; Schmidt, R.; Kuball, H.-G. Angewandte Chemie Int. Ed. 2008, 47, 4529-4532.

59. Reichardt, C. Solvents and Solvent Effects in Organic Chemistry, 3rd ed.; WileyVCH, 2003; Chapter 7: Empirical Parameters of Solvent Polarity.

60. Wohlfarth, C. Static Dielectric Constants of Pure Liquids and Binary Liquid Mixtures; Springer, 1991; Vol. 6.

61. Valeur, Molecular Fluorescence: Principles and Applications; Wiley-VCH, 2001.

62. Franck, J. Trans. Faraday Soc. 1926, 21, 536-542.

63. Condon, E. U. Physical Review 1928, 32, 858-872.

64. Kitai, A. Luminescent Materials and Applications; Wiley, 2008.

65. Lai, T.; Lim, E. Chem. Phys. Lett. 1979, 62, 507-510. 
66. Lim, E. C. J. Phys. Chem. 1986, 90, 6770-6777.

67. Lai, T.; Lim, B.; Lim, E. J. Am. Chem. Soc. 1982, 104, 7631-7635.

68. Gallagher, S. H.; Thompson, K. C.; Armstrong, R. S.; Lay, P. A. J. Phys. Chem. A 2004, 108, 5564-5572.

69. Lai, T.; Lim, E. Chem. Phys. Lett. 1980, 73, 244-248.

70. Wildanger, D.; Rittweger, E.; L., L. K.; Hell, S. Opt. Exp. 2008, 16, 9614-9621.

71. Kay, A. J.; Woolhouse, A. D.; Zhao, Y.; Clays, K. J. Mater. Chem. 2004, 14, 13211330. 